Klinik und Poliklinik für Augenheilkunde

Klinikum rechts der Isar

Technische Universität München

\title{
Messung der Akkommodation mittels optischer Biometrie
}

\author{
Aida Nurispahic
}

Vollständiger Abdruck der von der Fakultät für Medizin der Technischen Universität München zur Erlangung des akademischen Grades eines Doktors der Medizin genehmigten Dissertation.

Vorsitzender: $\quad$ Univ.-Prof. Dr. D. Neumeier

Prüfer der Dissertation:

1. apl. Prof. Dr. I. Lanzl

2. Univ.-Prof. Dr. Dr.(Lon.) Chr.-P. Lohmann

Die Dissertation wurde am 29.11.2007 bei der Technischen Universität München eingereicht und durch die Fakultät für Medizin am 07.05.2008 angenommen. 


\section{Inhaltsverzeichnis}

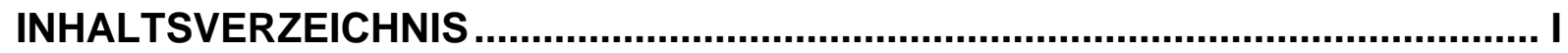

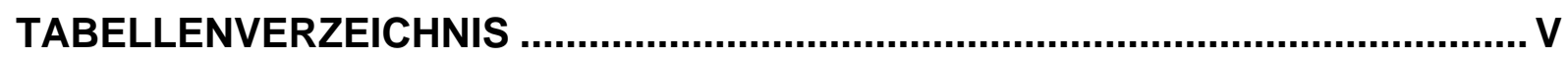

ABBILDUNGSVERZEICHNIS........................................................................ VII

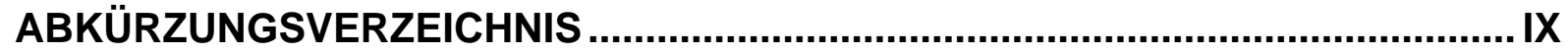

1 EINLEITUNG

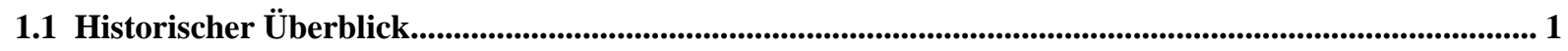

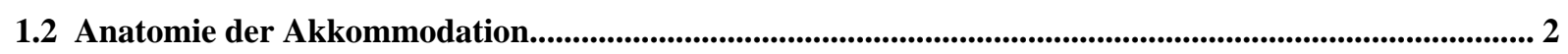

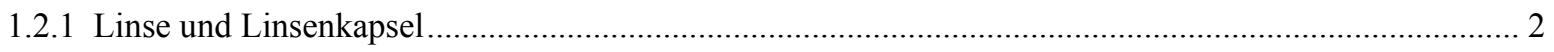

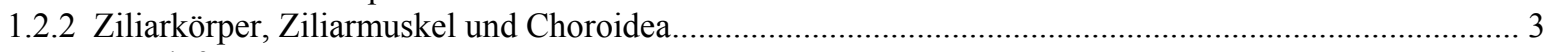

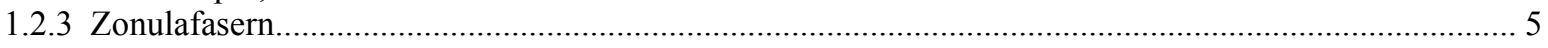

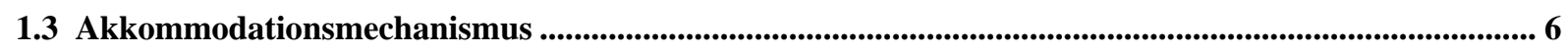

1.4 Veränderungen im Alter und Pathologien .................................................................................................. 8

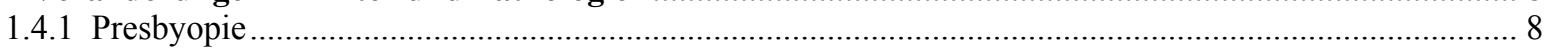

1.4.2 Akkommodationsspasmus und Akkommodationslähmung ………………….................................... 11

1.5 Stimulation und Messung der Akkommodationsleistung .............................................................................. 12

1.6 Kritische Würdigung der Literatur und Fragestellung................................................................................. 13

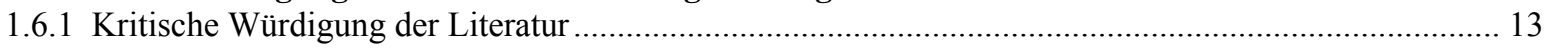

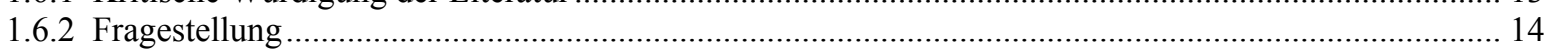

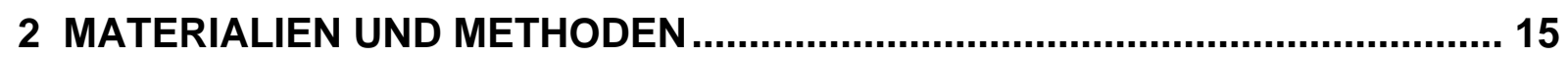

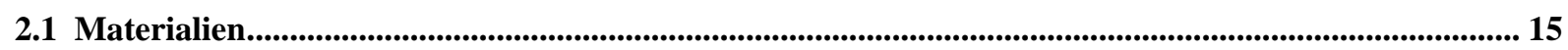

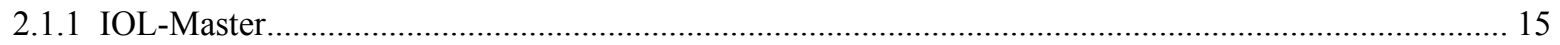

2.1.2 Zusatzeinrichtung zur physiologischen Stimulation der Akkommo- dation ......................................... 16

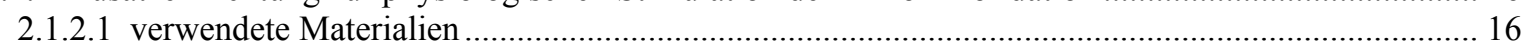

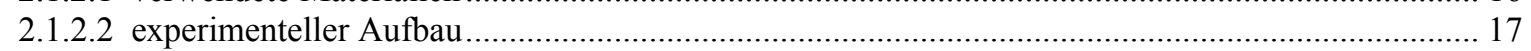

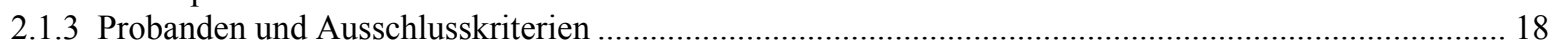

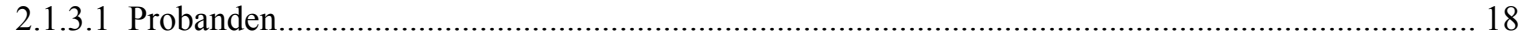

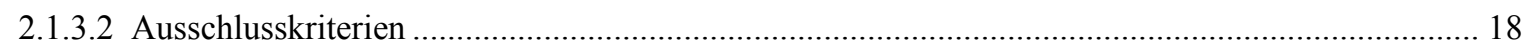

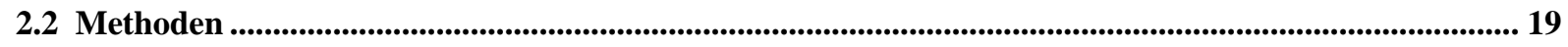

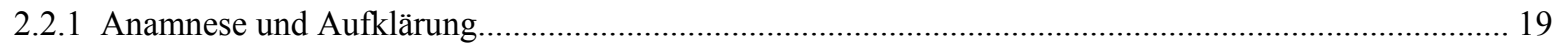

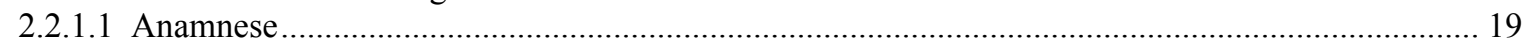

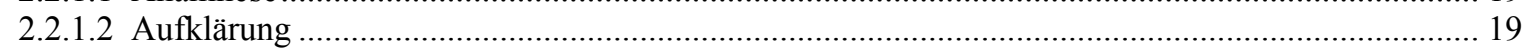

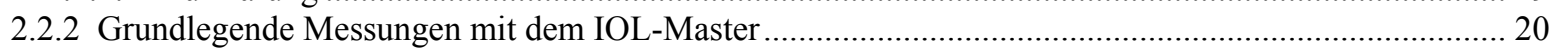

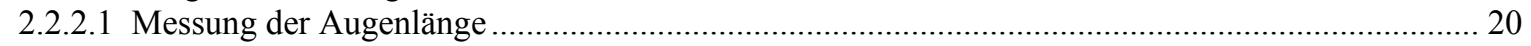

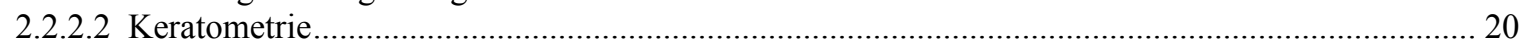

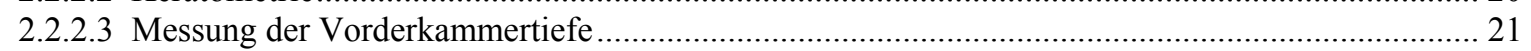

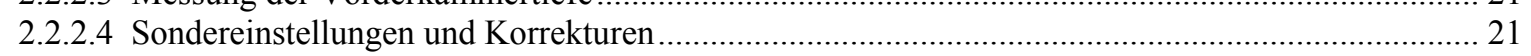

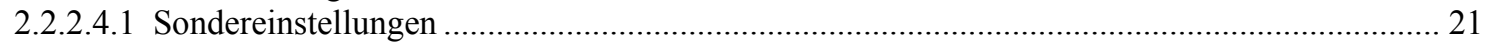

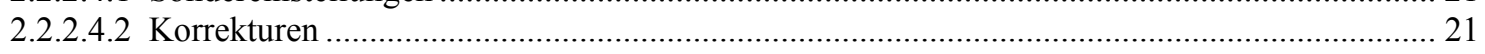




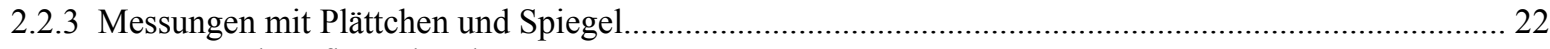

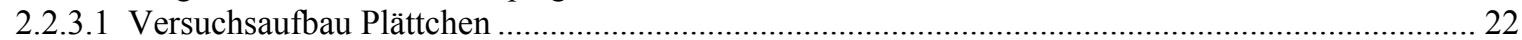

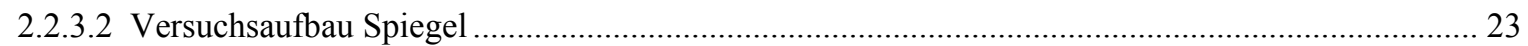

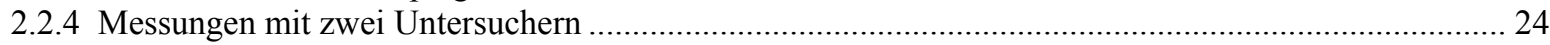

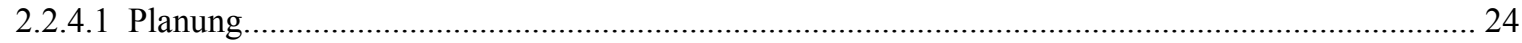

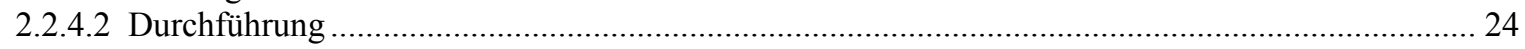

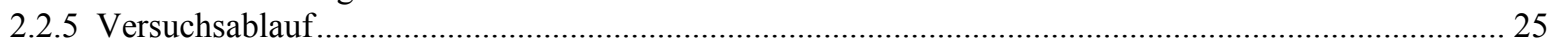

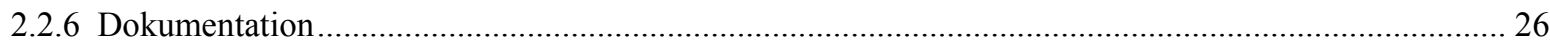

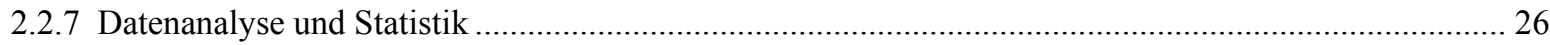

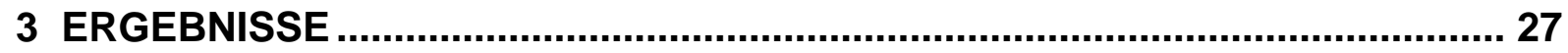

3.1 Beschreibung des Probandenkollektivs................................................................................................................ 27

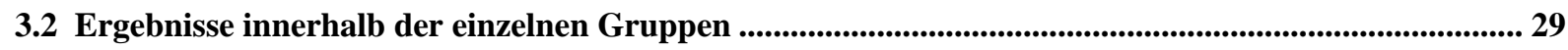

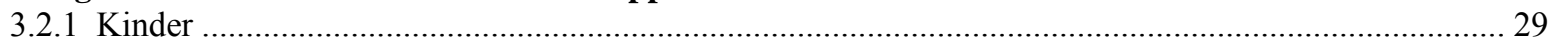

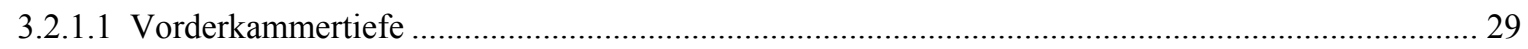

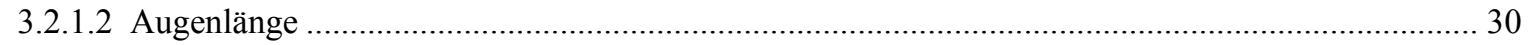

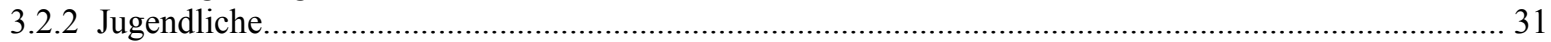

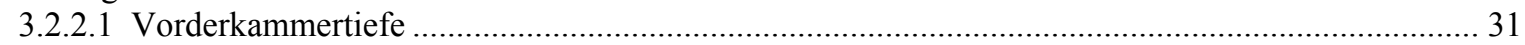

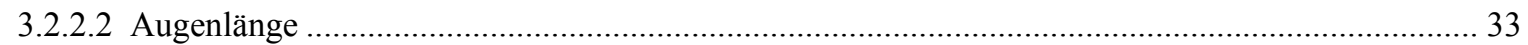

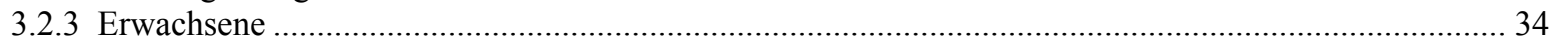

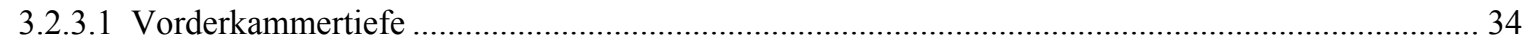

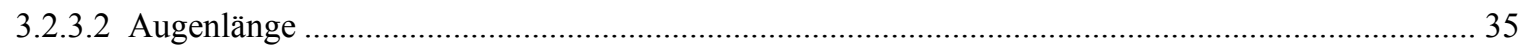

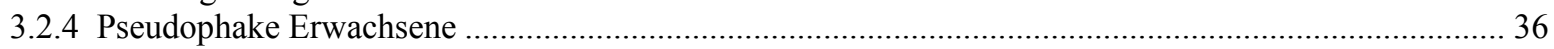

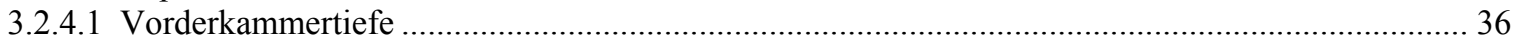

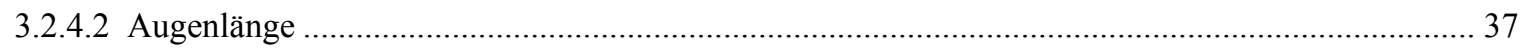

3.3 Veränderungen während der Akkommodation ..................................................................................37

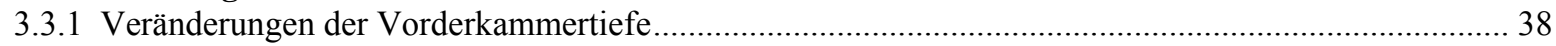

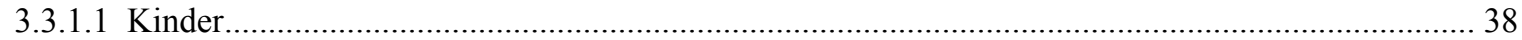

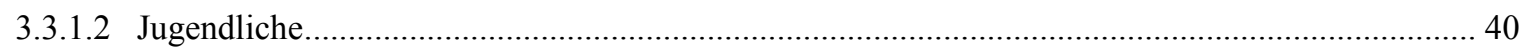

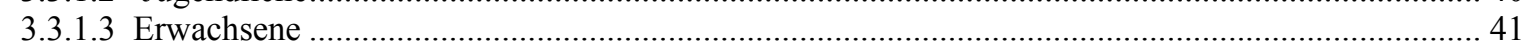

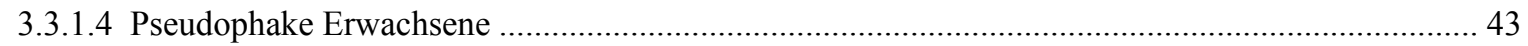

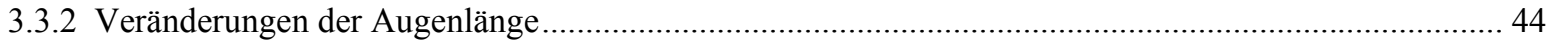

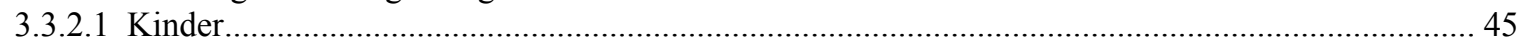

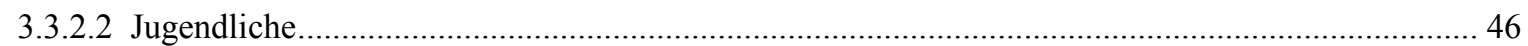

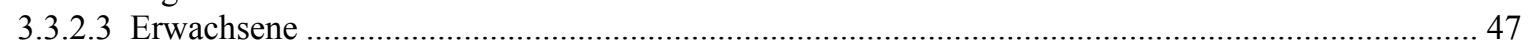

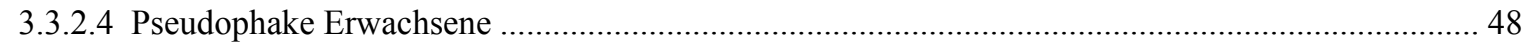

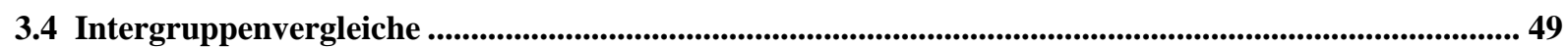

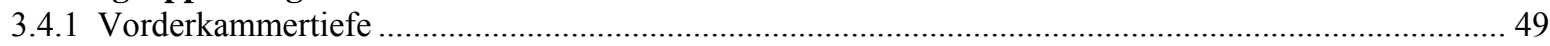

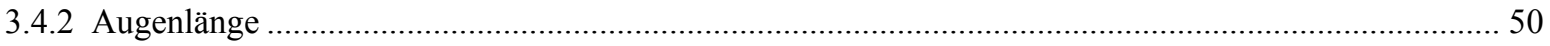

3.4.3 Veränderungen während der Akkommodation ..................................................................... 51

3.4.3.1 Veränderungen der Vorderkammertiefe ............................................................................. 51

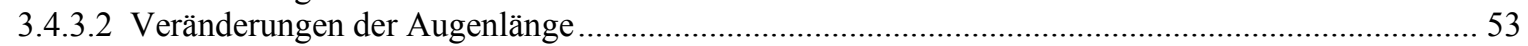

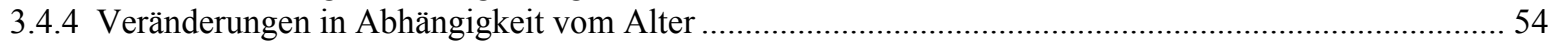

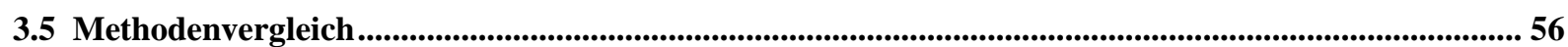

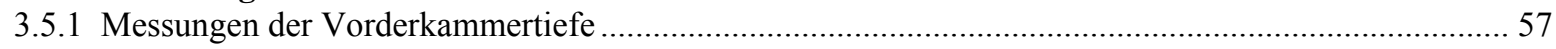

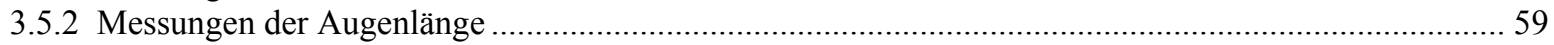

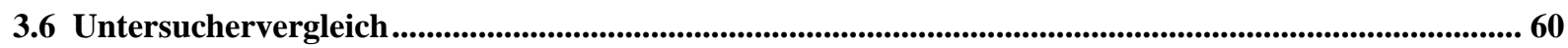

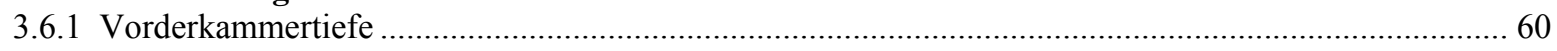

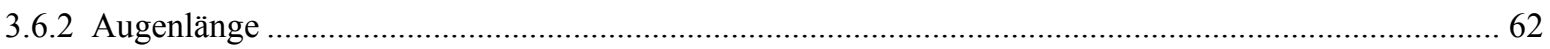

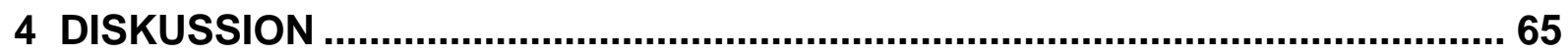

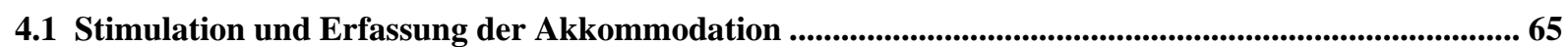

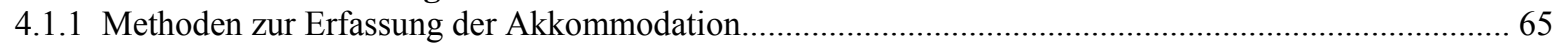


4.2 Statische Messungen: Vorderkammertiefe und Augenlänge ................................................................6 68

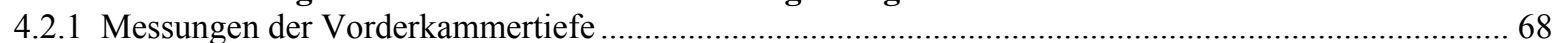

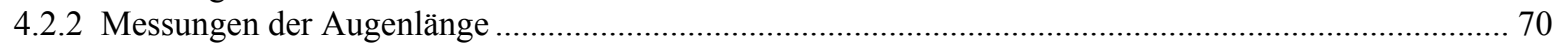

4.3 Augenparameter als Spiegel der Akkommodationsantwort ........................................................... 71

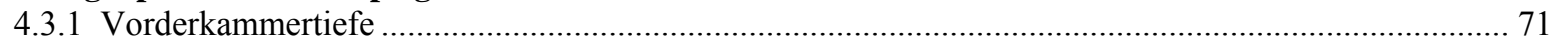

4.3.1.1 Abnahme der Vorderkammertiefe bei Nahakkommodation...................................................... 71

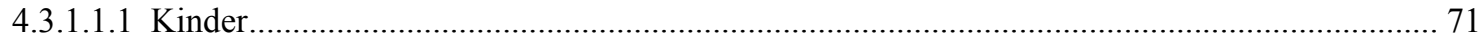

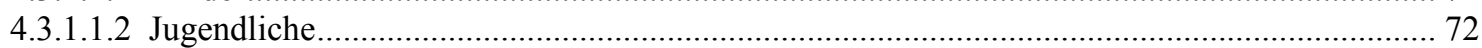

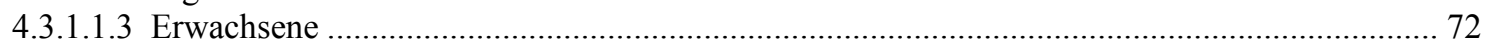

4.3.1.2 Änderung der Vorderkammertiefe bei Fernakkommodation .................................................... 73

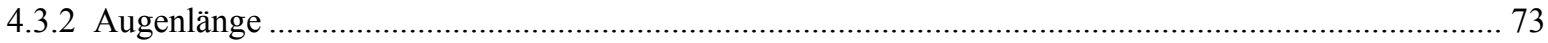

4.3.2.1 Änderung der Augenlänge bei Nahakkommodation ............................................................... 74

4.3.2.2 Rolle der Konvergenz bei Zunahmen der Augenlänge............................................................ 75

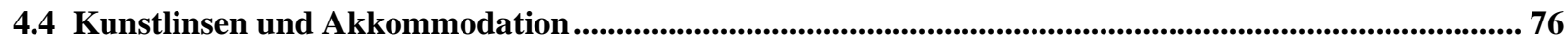

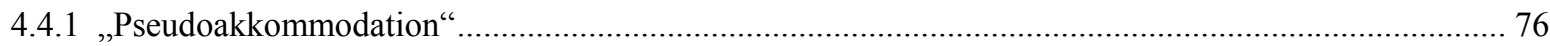

4.4.2 Änderung der Vorderkammertiefe bei Pseudophaken während der Nahakkommodation ..................... 76

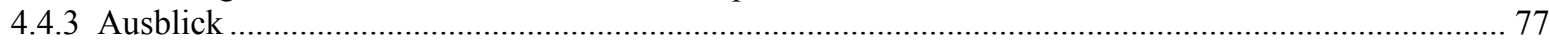

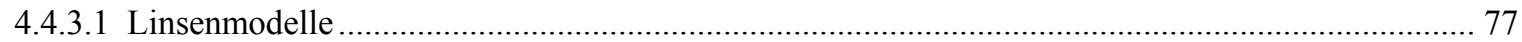

4.4.3.2 Alternativen zu Kunstlinsen: „Phako-Ersatz“ und skleraexpandierende Operationsverfahren ........ 78

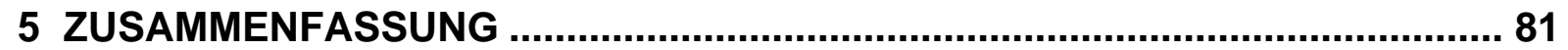

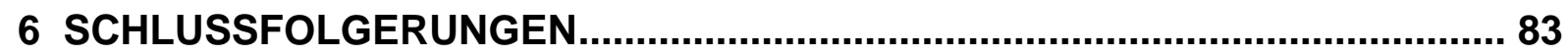

7 LITERATURVERZEICHNIS .................................................................... 85

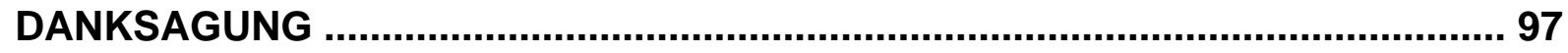

APPENDIX: PUBLIKATION „MESSUNG DER AKKOMMODATION MITTELS

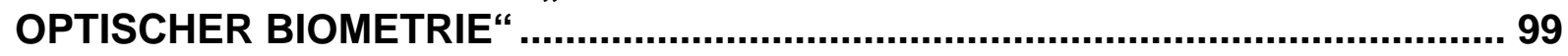

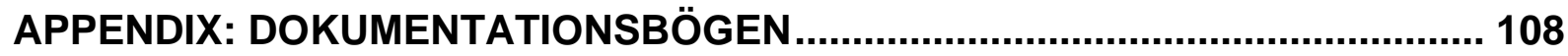




\section{TABELLENVERZEICHNIS}

Tabelle 1: Differentialdiagnosen bei Akkommodationslähmung und Akkommodationsspasmus, Auswahl nach Augustin (4, S.881).

Tabelle 2: Übersicht über Altersverteilung im Probandenkollektiv .......................................................... 28

Tabelle 3: Übersicht Linsen bei pseudophaken Probanden........................................................................... 28

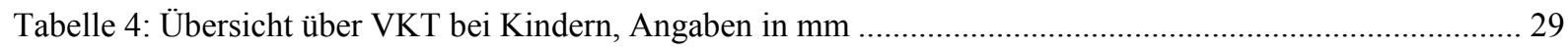

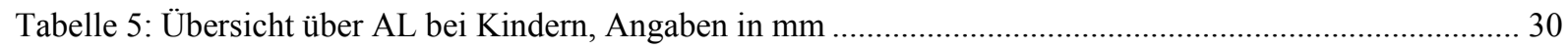

Tabelle 6: Übersicht über VKT bei Jugendlichen, Angaben in mm.............................................................. 32

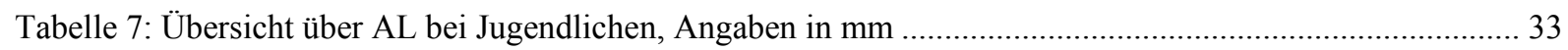

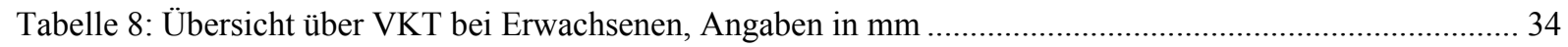

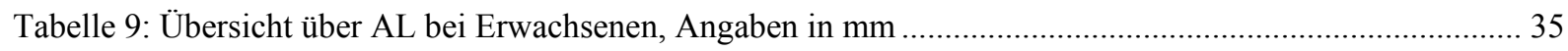

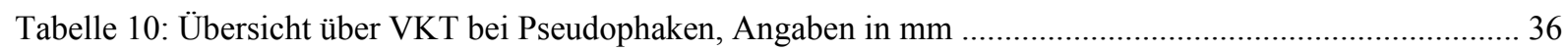

Tabelle 11: Übersicht über AL bei Pseudophaken, Angaben in $\mathrm{mm}$..................................................... 37

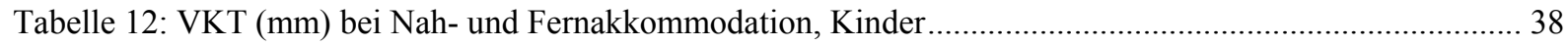

Tabelle 13: VKT (mm) bei Nah- und Fernakkommodation, Jugendliche ................................................. 40

Tabelle 14: VKT (mm) bei Nah- und Fernakkommodation, Erwachsene..................................................... 41

Tabelle 15: VKT (mm) bei Nah- und Fernakkommodation, Pseudophake .................................................. 43

Tabelle 16: AL (mm) bei Nah- und Fernakkommodation, Kinder......................................................... 45

Tabelle 17: AL (mm) bei Nah- und Fernakkommodation, Jugendliche.................................................... 46

Tabelle 18: AL (mm) bei Nah- und Fernakkommodation, Erwachsene ........................................................ 48

Tabelle 19: AL (mm) bei Nah- und Fernakkommodation, Pseudophake................................................... 48

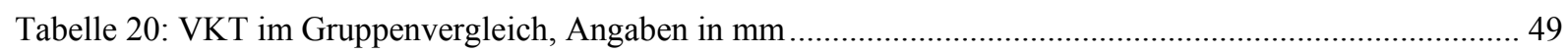

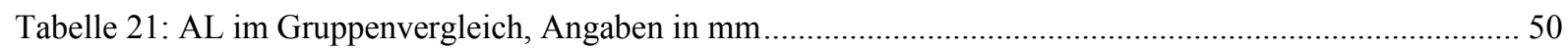

Tabelle 22: Differenz VKT (unakkommodiertes - nahakkommodiertes Auge, Methode: Plättchen) im Gruppenvergleich

Tabelle 23: Differenz AL (nahakkommodiertes - unakkommodiertes Auge, Methode: Plättchen) im Gruppenvergleich.

Tabelle 24: Korrelation zwischen den Methoden bei Messung der VKT ......

Tabelle 25: Differenz VKT (unakkommodiertes - nahakkommodiertes Auge) im Methodenvergleich, Angaben in $\mathrm{mm}$ 
Tabelle 26: Korrelation zwischen den Methoden bei Messung der AL

Tabelle 27: Differenz AL (nahakkommodiertes- unakkommodiertes rechtes Auge) im Methodenvergleich bei Jugendlichen, Angaben in mm

Tabelle 28: Untersuchervergleich bei Anwendung zweier Methoden 60

Tabelle 29: Korrelation zwischen den Untersuchern bei Messung der VKT. 61

Tabelle 30: Korrelation zwischen den Untersuchern bei Messung der AL 


\section{ABBILDUNGSVERZEICHNIS}

Abbildung 1: Anatomie des Akkommodationsapparats (79, S.56) ........................................................ 2

Abbildung 2: akkommodativer Mechanismus nach Helmholtz (abgeändert nach 31, S.209)........................... 6

Abbildung 3: Änderung der Akkommodationsbreite mit dem Alter (abgeändert nach 67, S.305) ...................... 9

Abbildung 4: schematische Darstellung der Zusatzeinrichtung zur physiologischen Stimulation

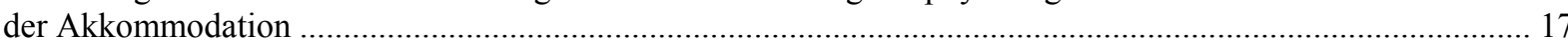

Abbildung 5: fotografische Darstellung des Versuchsaufbaus....................................... 17

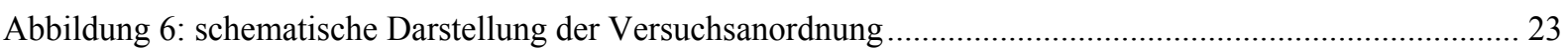

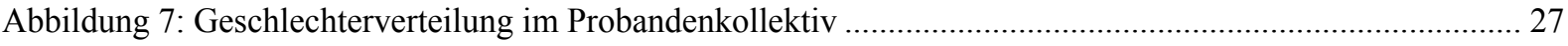

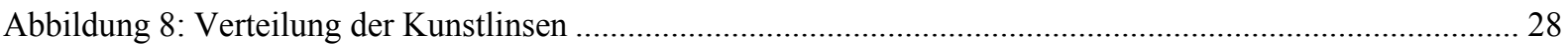

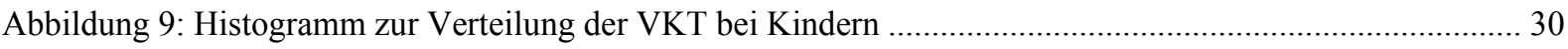

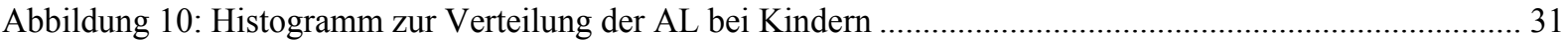

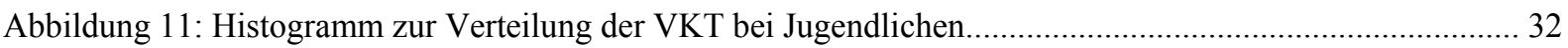

Abbildung 12: Histogramm zur Verteilung der AL bei Jugendlichen .................................................. 33

Abbildung 13: Histogramm zur Verteilung der VKT bei Erwachsenen ......................................................... 34

Abbildung 14: Histogramm zur Verteilung der AL bei Erwachsenen ................................................... 35

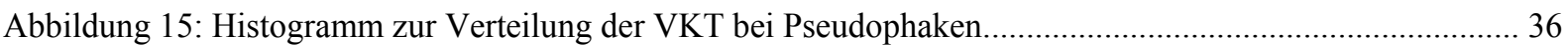

Abbildung 16: Histogramm zur Verteilung der AL bei Pseudophaken ..................................................... 37

Abbildung 17: Säulendiagramm zur Differenz VKT

(unakkommodiertes - nahakkommodiertes rechtes Auge), Kinder ............................................................ 39

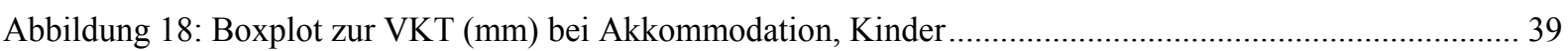

Abbildung 19: Säulendiagramm zur Differenz VKT

(unakkommodiertes - nahakkommodiertes rechtes Auge), Jugendliche .................................................... 40

Abbildung 20: Boxplot zur VKT (mm) bei Akkommodation, Jugendliche ................................................... 41

Abbildung 21: Säulendiagramm zur Diferenz VKT

(unakkommodiertes - nahakkommodiertes rechtes Auge), Erwachsene ................................................... 42

Abbildung 22: Boxplot zur VKT (mm) bei Akkommodation, Erwachsene..................................................... 42

Abbildung 23: Säulendiagramm zur Differenz VKT

(unakkommodiertes - nahakkommodiertes rechtes Auge), Pseudophake .................................................... 43

Abbildung 24: Boxplot zur VKT (mm) bei Akkommodation, Pseudophake................................................. 44

Abbildung 25: Fehlerbalken zur AL bei Akkommodation, alle Probanden ............................................... 45

Abbildung 26: Säulendiagramm zur Differenz AL 
(nahakkommodiertes - unakkommodiertes rechtes Auge), Kinder....

Abbildung 27: Säulendiagramm zur Differenz AL

(nahakkommodiertes - unakkommodiertes rechtes Auge), Jugendliche

Abbildung 28: Säulendiagramm zur Differenz AL

(nahakkommodiertes - unakkommodiertes rechtes Auge), Erwachsene....

Abbildung 29: Säulendiagramm zur Diferenz AL

(nahakkommodiertes - unakkommodiertes rechtes Auge), Pseudophake

Abbildung 30: Boxplot zur Verteilung der VKT im Gruppenvergleich

Abbildung 31: Boxplot zur Verteilung der AL im Gruppenvergleich

Abbildung 32: Boxplot zur Verteilung der Differenz VKT (unakkommodiertes - nahakkommodiertes Auge,

Methode: Plättchen) im Gruppenvergleich

Abbildung 33: Boxplot zur Verteilung der Differenz AL (nahakkommodiertes - unakkommodiertes Auge, Methode: Spiegel) im Gruppenvergleich ....

Abbildung 34: Zusammenhang Alter und VKT, Kinder und Jugendliche

Abbildung 35: Zusammenhang Alter und VKT, Jugendliche und Erwachsene

Abbildung 36: Zusammenhang Alter und Akkommodationsantwort, gemessen in der Differenz der VKT ......

Abbildung 37: Zusammenhang Alter und AL

Abbildung 38: Änderung der VKT bei Nahakkommodation (Angaben in mm) im Methodenvergleich

Abbildung 39: Differenz VKT (unakkommodiertes - nahakkommodiertes Auge, Angaben in mm) im Methodenvergleich.

Abbildung 40: AL bei Nahakkommodation (Angaben in $\mathrm{mm}$ ) im Methodenvergleich.

Abbildung 41: VKT bei Nahakkommodation im Untersuchervergleich.....

Abbildung 42: Diferenz VKT(unakkommodiertes - nahakkommodiertes Auge) im Untersuchervergleich

Abbildung 43: AL bei Nahakkommodation, Methode: Plättchen, im Untersuchervergleich.

Abbildung 44: AL bei Nahakkommodation, Methode: Spiegel, im Untersuchervergleich 


\section{ABKÜRZUNGSVERZEICHNIS}

\begin{tabular}{|c|c|}
\hline Abkürzung & Begriff \\
\hline$\overline{\mathrm{AL}}$ & Augenlänge \\
\hline $\mathrm{dpt}$ & Dioptrie(n) \\
\hline IOL & Intraokularlinse \\
\hline LED & light emitting diode \\
\hline M. & Musculus \\
\hline Max & Maximum \\
\hline Min & Minimum \\
\hline MW & Mittelwert \\
\hline $\mathrm{N}$ & Anzahl \\
\hline N. & Nervus \\
\hline PCI & Partielle Kohärenz-Interferometrie \\
\hline PMMA & Polymethylmethacrylat \\
\hline $\mathrm{r}$ & Korrelationskoeffizient \\
\hline SD & Standardabweichung \\
\hline VKT & Vorderkammertiefe \\
\hline
\end{tabular}




\section{Einleitung}

\subsection{Historischer Überblick}

Unter Akkommodation versteht man die Fähigkeit der Augenlinse, ihre Brechkraft so zu verändern, dass sowohl nahe als auch weit entfernte Sehobjekte auf der Netzhaut scharf abgebildet werden können. (84, S. 334)

Die ersten Versuche auf diesem Gebiet wurden von Christoph Scheiner im Jahre 1619 beschrieben, der eindrucksvoll bewies, dass das Auge über einen gut funktionierenden Mechanismus verfügt, die Lage seines Fokus zu ändern. Durch die Arbeiten von Thomas Young am Anfang des 19. Jahrhunderts wurde jedoch zum ersten Mal deutlich, dass nicht, wie von seinen Vorgängern zunächst angenommen, in erster Linie Veränderungen der Kornea oder der Augenlänge, sondern in der Tat die der Linse für die Akkommodationsfähigkeit verantwortlich sind. (67, S.294) Die Beschreibung des Ziliarmuskels gelang schließlich Crampton 1813 und wurde von Müller 1857 um die Theorie, wie dieser die Linsenkrümmung bei Vögeln beeinflussen könnte, ergänzt. (31, S.197)

Der lange Weg zum Verständnis der menschlichen Akkommodation wurde in erster Linie durch Studien an verschiedenen Tierarten gebahnt.

Heute wissen wir, dass bei Wirbeltieren der Akkommodationsmechanismus stark von der Abstammungsgeschichte, aber auch vom Lebensraum und vom Ernährungsmodus abhängt. Pflanzenfresser, bzw. Tiere, die auf Futtersuche vorwiegend auf ihren Geruchssinn zurückgreifen müssen, scheinen auch die Fähigkeit zur Akkommodation kaum entwickelt zu haben.

Kraftvolle Mechanismen zur Veränderung der Linsenkrümmung, um in der Nähe scharf sehen zu können, findet man hingegen bei Vögeln und Reptilien. (30, S.253) Zum Teil wird die Linse als Ganzes nach vorne (z.B. bei Amphibien) oder nach hinten (z.B. Fische) verschoben. $(79$, S. 51)

Die größten Akkommodationsamplituden weisen amphibische Vertebraten, also Tiere, die sowohl auf Wasser als auch auf dem Land leben, wie z.B. Tauchvögel, auf. So haben Tauchenten eine Akkommodationsbreite bis hin zu 80 Dioptrien, während im Vergleich das Maximum beim jungen Menschen bei etwa 15 Dioptrien liegt. (31, S. 198) 


\subsection{Anatomie der Akkommodation}

Am Akommodationsmechanismus beteiligt sind die Linse, die Linsenkapsel, der Ziliarkörper, der Ziliarmuskel, die Choroidea, sowie anteriore und posteriore Zonulafasern.

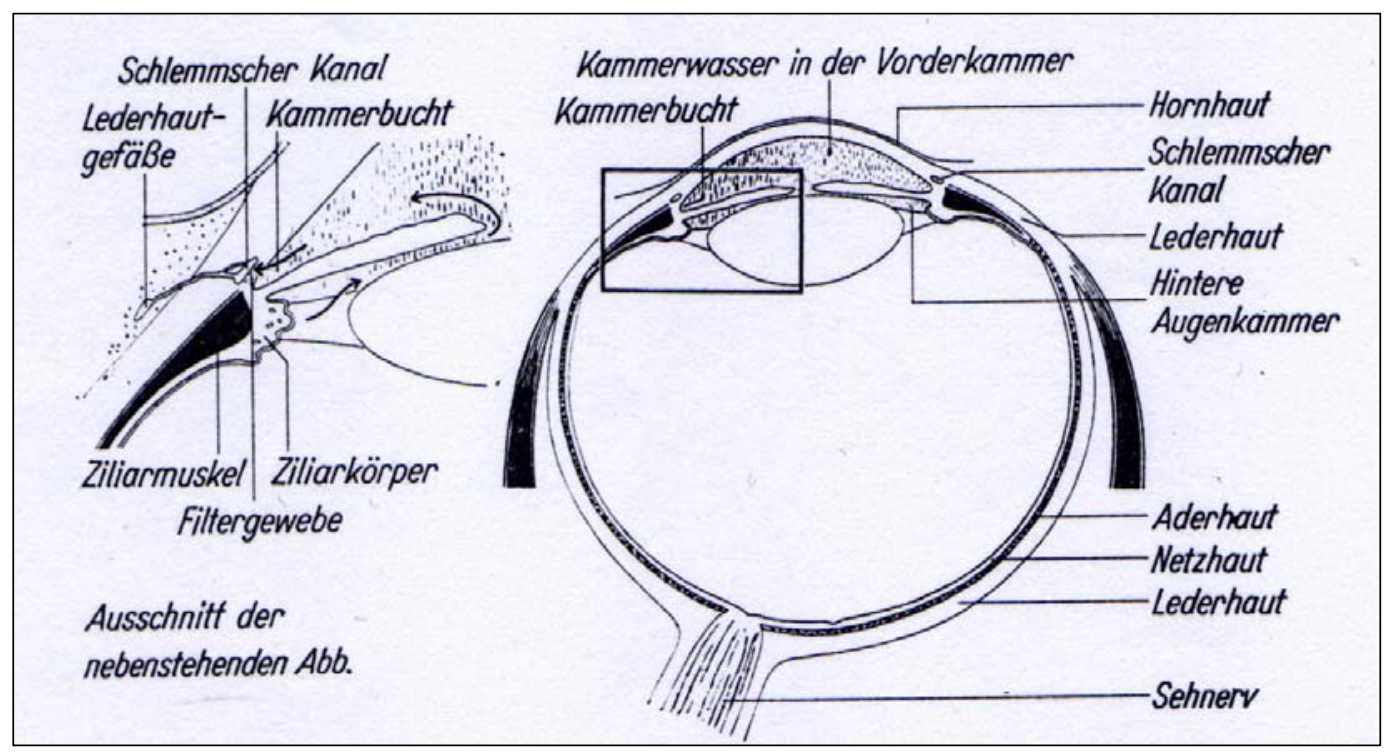

Abbildung 1: Anatomie des Akkommodationsapparats (79, S.56)

\subsubsection{Linse und Linsenkapsel}

Die Entstehung der Linse aus dem Ektoderm wird durch die nach vorne wachsende Augenblase induziert. Das Ektoderm wird an der Stelle zunächst mehrschichtig (Linsenplakode), senkt sich aber später erst als Grube (Fovea lentis) und in der dritten embryonalen Lebenswoche dann als Bläschen (Vesicula lentis) ab. Das primitive Linsenblasenepithel gibt nach außen hin eine Basalmembran ab, die dann zur Linsenkapsel wird. (78, S.723) Ihr Gewicht nimmt altersabhängig fast um das fünffache zu: während sie bei Geburt ein Gewicht von $65 \mathrm{mg}$ hat, wiegt sie bei einem 20-jährigen bereits $152 \mathrm{mg}$ und beim 80 -jährigen sogar etwa $258 \mathrm{mg}$. (95, S.453)

Sie hat eine bikonvexe Form und ist hinten stärker gekrümmt als vorne. Die Linse ist in der Fossa patellaris des Glaskörpers eingebettet und sie wird von den Zonulafasern, die eine elastische Eigenspannung besitzen, im Zentrum der hinteren Augenkammer gehalten und vor Erschütterungen und Dislokation geschützt. Ihr vorderer Pol ragt in die Vorkammer. Sie ist allseits von Kammerwasser umgeben, was in sofern von großer Bedeutung ist, da das gefäßund nervenfreie Organ von diesem durch Diffusion oder aktive Transportmechanismen ernährt wird, wobei sie den Großteil der Energie aus der Glykolyse gewinnt. (39, S. 458) 
Grundsätzlich können wir mikroskopisch an der Linse Kapsel, subkapsuläres Epithel und Linsenfasern unterscheiden. Die elastische und widerstandsfähige Kapsel ist zwar homogen, jedoch vorne dicker $(11$ bis $19 \mu \mathrm{m})$ als an der Rückfläche (4 bis $5 \mu \mathrm{m})$. (79, S.55) Sie ist an zwei zum Äquator hin konzentrischen Zonen, an denen die Zonulafasern einstrahlen, verdickt. Unter der vorderen Kapsel befindet sich das kubisch bis zylindrische, einschichtige Linsenepithel. Man muss von der ruhenden zentralen Zone des Linsenepithels die germinativ aktive Peripherie unterscheiden, da diese lebenslang sekundäre Linsenfasern bildet, die sich schalenartig auf die alten auflagern (appositionelles Wachstum). (9, S.117; 85, S.146) Anatomisch stellen die Linsenfasern die Linsenrinde dar. Da in der Linse im Gegensatz zu anderen epithelialen Organen keine Zellabstoßung statt findet, kann man sich leicht erklären, warum sie zeitlebens kontinuierlich wächst.

Am Äquator geht das Linsenepithel durch bipolares Wachstum in immer länger werdende Linsenfasern über. Diese reichen zu Beginn noch von Pol zu Pol, verlängern sich mit der Zeit jedoch im Vergleich zum Wachstum der Oberfläche deutlich weniger, was dazu führt, dass Lücken auftreten, die von einer Kittsubstanz ausgefüllt werden. So entstehen Linsensterne. Die später gebildeten Linsenfasern vereinigen sich bereits auf komplexeren Nahtlinien, so dass Linsensterne bei Erwachsenen in der Regel schon sechs bis zehn Hauptarme und zehn bis sechzehn Nebenarme aufweisen. (79, S.53)

Durch Abnahme des Wassergehaltes, Verdichtung und Zunahme des spezifischen Gewichtes entsteht im Alter ein sich vergrößernder und verhärtender Kern, wohingegen der Rindenanteil abnimmt. (85, S.146) Diese so genannte Linsensklerose, die mit einem Verlust an Flexibilität und Elastizität einhergeht, ist mitverantwortlich für die Entwicklung der Presbyopie.

Neben der Tatsache, dass die transparente Linse also entscheidend zur Brechkraft des Auges beiträgt (10-20dpt), besitzt sie eine protektive Filterwirkung für langwellige UV-Strahlen zwischen 300 und $400 \mathrm{~nm}$. (85, S.147)

\subsubsection{Ziliarkörper, Ziliarmuskel und Choroidea}

Entwicklungsgeschichtlich entspringen der Ziliarmuskel und das Stroma des Ziliarkörpers aus dem Mesenchym der Kopfganglienleiste, die beiden Epithelschichten gehen jedoch aus dem Augenbecher hervor. Das innere, unpigmentierte Epithel ist mit seiner basalen Oberfläche zur Augenhinterkammer, mit der apikalen aber zum Pigmentepithel orientiert. (78, S.719) Beim Ziliarkörper handelt es sich um einen kranzförmigen Abschnitt des Auges, der sich von der Iriswurzel und dem Skleralsporn bis zur Ora serrata, an welcher ein scharfer Übergang in die Retina besteht, erstreckt. Seine hintere Partie ist flach (Pars plana), wohingegen der 
vordere Abschnitt Falten aufweist und daher als Pars plicata bezeichnet wird. (78, S. 719) Den Großteil des Ziliarkörpers nimmt der M. ciliaris ein, an dem man der klassischen Histologie nach einen äußeren longitudinalen (Brückescher Anteil), einen inneren zirkulären (Müllerscher Abschnitt) und eine dazwischen gelegene radiäre oder retikuläre Partie unterscheidet. Sie stellen jedoch ein zusammenhängendes funktionelles System dar. Der Ziliarmuskel ist ungewöhnlich reich innerviert und kontrahiert sich somit sehr schnell, wodurch er sich von anderen glatten Muskeln unterscheidet. Interessant ist, dass man bei Vögeln an dieser Stelle tatsächlich einen quergestreiften Muskel vorfindet. (31, S.203)

Wir finden sowohl sympathische Anteile, die ihre Wirkung über B2- Rezeptoren geltend machen, als auch eine dominante parasympathische Innervation, die über muskarinische M3Rezeptoren vermittelt wird. Befestigt ist der Ziliarmuskel in fünffach verschiedener Weise an der Korneosklera und Uvea, was bei Kontraktion als fester vorderer Anker dient.

Nach hinten strahlen die Sehnen büschelartig in die Bruchsche Membran ein (hintere Fixation), welche aus zwei elastischen Netzen, longitudinal und äquatorial angeordnet, besteht. Neben der Aufrechterhaltung des intraokularen Druckes und der Bildung der BlutRetina-Schranke, hat die Bruchsche Membran die Aufgabe, das Einwachsen von Choroidalgefäßen in das Pigmentepithel zu verhindern und die Desakkommodation zu vermitteln. (78, S.723) Kontrahiert sich der Muskel, so wird dieses elastisch-muskulöse System gespannt, speichert also Energie, so dass bei Dilatation kein Antagonist notwendig ist. (79, S.53) Mit Anspannung des Muskels zieht er also als Ganzes die anteriore Choroidea nach vorne und reduziert so den Zug an den Zonulafasern. Dabei findet eine Vergrößerung des zirkulären Muskelanteils auf Kosten der retikulären und longitudinalen Fasergruppen statt. (31, S. 203) Auffällig ist, dass der Ziliarmuskel nicht in jedem Lebensalter die gleiche Form aufweist. Bei Geburt ist seine Entwicklung noch nicht vollständig abgeschlossen, erst mit 15 Jahren ist er voll differenziert, besteht aber noch überwiegend aus longitudinalen und radiären Fasern. Während zunächst einmal bis zum 35. Lebensjahr sowohl Bindegewebe als auch Muskulatur an Masse zunehmen, degenerieren die Muskelfasern mit steigendem Alter und werden durch kollagenes Bindegewebe ersetzt. Vom 55. Lebensjahr an kann man bereits von einem senil-atrophischen Ziliarmuskel sprechen. (79, S.63)

Es ist natürlich nicht zu vergessen, dass der Ziliarkörper neben der Akkommodation noch einige weitere Aufgaben erfüllt. Sein Epithel sezerniert das Kammerwasser, das für die Ernährung der Linse und der Hornhaut, sowie die Aufrechterhaltung des intraokularen Druckes unabdingbar ist. (85, S.175) Darüber hinaus ist er am Aufbau des Halteapparates der Linse beteiligt und produziert zusätzlich die Hyaluronsäure für den Glaskörper. (78, S.719) 


\subsubsection{Zonulafasern}

Die Zonulafasern (Zonula ciliaris Zinni) treten zum ersten Mal im dritten Schwangerschaftsmonat in Erscheinung. Ihr Bildungsort ist das unpigmentierte Ziliarepithel. (78, S.725) Sie befinden sich zwischen Linse, Ziliarkörper und Glaskörper. Während der Großteil des Zonulaapparates seinen Ursprung am Pars plana des Ziliarkörpers, am sogenannten Orbicus ciliaris, hat, entspringen einige wenige Fasern direkt vom Glaskörper oder von den Spitzen der Ora serrata. (79, S.65)

Die Fasern bestehen aus Fibrillen mit einem Durchmesser von 70 bis $80 \mathrm{~nm}$, die sich zu größeren Bündeln mit einer schwankenden Größe von etwa 4 bis $50 \mu \mathrm{m}$ gruppieren. Da sich die Techniken und die Terminologie, mit denen versucht wurde, die Zonulafasern darzustellen, weitgehend unterscheiden, variieren auch ihre Beschreibungen. (31, S.203) Grundsätzlich unterscheidet man nach Rohen zwei funktionell unterschiedliche Anteile von Zonulafasern: Halte- und Spannfasern. (80, S.133) Die Haltefasern sind kräftige Bündel, die sich vom Orbiculus ciliaris durch die Ziliartäler hindurch bis zur Linse ziehen. Die kürzeren Zug- oder Spannfasern, die unter spitzem Winkel von Haltefasern abzweigen, ziehen schräg nach vorne zum Ziliarepithel bis zur Höhe der Ziliarmuskelkante, bevor sie sich dort in feinste Faserfibrillen aufzweigen. Diese setzen weitflächig an, vom hinteren Ende des Ziliarmuskels bis zur Muskelkante. (79, S.66)

Das Hauptfaserbündel zieht also zwischen die Ziliarfalten, wobei es sich in deren Basalmembran in Form flacher Bänder verankert. In Bereich dieser so genannten Ziliartäler teilt es sich auf (Zonulagabel), zieht dann größtenteils in der Nähe des Äquators an die Vorder- und Rückseite der Linse und strahlt schließlich in Gestalt von Mikrofibrillen oberflächlich in die Linsenkapsel ein. (78, S.725)

Als Aufhängeapparat der Linse haben Zonulafasern die Aufgabe, jene zu stabilisieren und den Akkommodationsvorgang zu ermöglichen. Durch den nach außen gerichteten Zug der an der äquatorialen Linsenregion angehefteten anterioren Zonula wird die Kapsel gespannt und die Linse somit in ihrer flachen unakkommodierten Position gehalten. Obwohl man morphologisch mit den Jahren keine Veränderung an den Zonulafasern nachweisen kann, werden diese zunehmend spröder und zerreißen im Alter leichter als bei Jugendlichen. (78, S.725) 


\subsection{Akkommodationsmechanismus}

All die im vorigen Kapitel beschriebenen Strukturen können dazu beitragen, dass der Brechungszustand des Auges verändert werden kann.

Wenn ein emmetropes Auge einen weit entfernten Gegenstand betrachtet, ist es im Sprachgebrauch unakkommodiert. In diesem Zustand wird die Linse durch die Zonulafasern, die, wie bereits erörtert, am Linsenäquator eine nach außen gerichtete Spannung aufbauen, in einer flachen Position gehalten. Dabei ist der Ziliarmuskel erschlafft und die Bruchsche Membran der Choroidea zieht den Ziliarkörper nach hinten, wobei die dort angehefteten Zonulafasern diese Spannung auf den Zonulaapparat und die Linsenkapsel übertragen.

Sobald man jedoch den Versuch unternimmt, auf ein in der Nähe gelegenes Objekt zu fokussieren, finden am Auge einige Veränderungen statt.

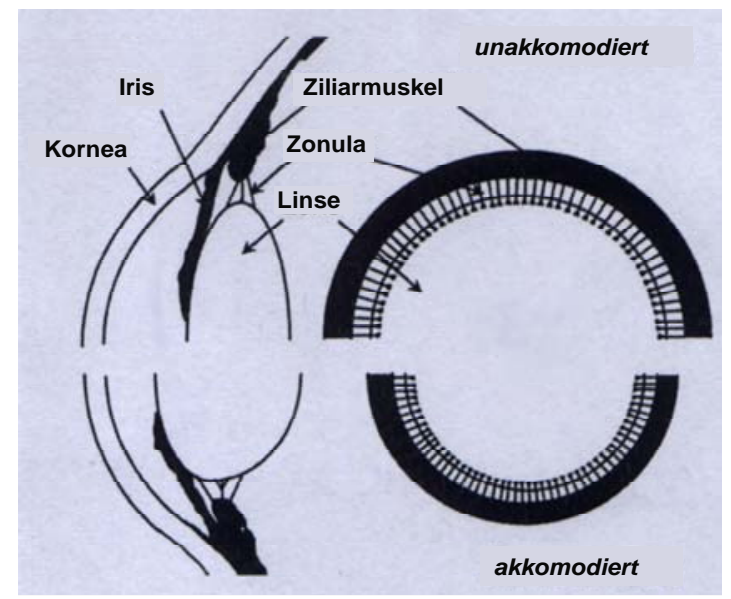

Abbildung 2: akkommodativer Mechanismus nach Helmholtz (abgeändert nach 31, S.209)

- Zum einen kommt es zu einer Kontraktion der Pupille, welche sowohl bei Akkommodation, als auch bei Konvergenz beschrieben ist.

- Darüber hinaus spannt sich der Ziliarmuskel an und die innere Spitze des Ziliarkörpers bewegt sich nach vorne und in Richtung der Augenachse (axipetal), wobei sich der zirkuläre Muskelanteil auf Kosten des retikulären und longitudinalen vermehrt. Dies führt dazu, dass sich die Anheftungsflächen der Zonulafasern am Ziliarkörper in Richtung der Linsenäquators schieben, womit die Spannung der Zonulafasern gelockert wird. So kann die Linsenkapsel die Linse in eine kugelförmige, rundere Form drücken. (79, S.68)

Diese besondere Bedeutung der Linsenkapsel wurde erst Mitte des 20.Jahrhunderts durch Fincham hervorgehoben, während wir die Erklärung des prinzipiellen Aufbaus des 
Akkommodationsvorganges vorwiegend Helmholtz und Gullstrand (Anfang des 20. Jahrhunderts) zu verdanken haben. (67, S.298)

- Der anteriore Linsenpol bewegt sich dabei nach vorne und nimmt die Aderhaut mit. Diese Bewegung hat eine Abnahme der Vorderkammertiefe zur Folge. (5, S.1843)

- Entgegengesetzt der ursprünglichen Meinung, dass sich die posteriore Linsenfläche bei der Akkommodation in keinster Weise verändert, wird ihr inzwischen eine größere Rolle zugeschrieben. Es konnte bisher eine Zunahme der Krümmung und eine damit einhergehende Rückwärtsbewegung der hinteren Linsenfläche während der Akkommodation nachgewiesen werden. (29, S.12; 7, S.523; 51, S.1141)

- Darüber hinaus wird die vordere Linsenfläche zu einem deutlich höheren Grade als die hintere konvexer, die axiale Linsendicke nimmt zu und der Linsendurchmesser sinkt. $(22$, S.117; 23, S.2363)

- Durch die Rückwärtsbewegung der hinteren Linsenfläche nimmt die Tiefe des Glaskörperraumes leicht ab.

- Außerdem sinkt die Linse während der Akkommodation durch die Schwerkraft leicht nach unten. $(67$, S.297)

Während also die Linsenkapsel die größte Kraft im Akkommodationsvorgang aufzuwenden hat, scheint die Linsensubstanz das Ausmaß der Krümmung sogar einzudämmen.

Im Zusammenhang mit dem Akkommodationsmechanismus wird auch Überlegungen nachgegangen, die dem Glaskörper eine größere Bedeutung zuschreiben (15, S.1063), jedoch gibt es bei den bisherigen Publikationen dazu noch keine klare Linie.

All diese beschriebenen Vorgänge führen also zu einer Brechkraftzunahme der Linse.

Wird jedoch nicht mehr auf den nahen Gegenstand fokussiert, erschlafft der Ziliarmuskel und wird durch die in der elastischen Lamelle der Bruchschen Membran gespeicherte Energie in den ursprünglichen, unakkommodierten Zustand zurückverlagert. Dieses wiederum spannt die anterioren Zonulafasern am Linsenäquator an und zieht die Linse wieder flach.

Interessant ist, dass unsere Augen bereits in Ruhe, also bei Fehlen jeglichen Akkommodationsimpulses, eine Myopie von - 0,5 bis hin zu -4,0 dpt aufweisen. (84, S.335) Wird das Auge also stimuliert, reagiert es, wie bereits beschrieben, mit Pupillenkonstriktion, Konvergenz und Verstärkung der Linsenkrümmung. Diese drei Handlungen werden von 
präokzipital gelegenen Zentren der primären Sehrinde gesteuert. Der efferente Schenkel führt zunächst zur Area praetectalis, wo die Umschaltung auf ein zweites Neuron erfolgt, das zum Ncl. oculomotorius accessorius (Edinger-Westphal) projiziert. Von hier aus ziehen präganglionäre parasympathische Fasern über den N. oculomotorius zum Ganglion ciliare, wo es zur Umschaltung auf postganglionäre Neurone kommt, deren Axone M. ciliaris und M. sphincter pupillae innervieren. Die für die Konvergenz verantwortlichen Mm. recti mediales werden ebernfalls über die Kerne des Okulomotoriuskomplexes innerviert. (78, S.747) Wenngleich ein akkommodativer Stimulus lediglich einem Auge präsentiert wird, kommt es, ähnlich wie bei der konsensuellen Pupillenreaktion, zur Naheinstellung und Konvergenz beider Augen. (31, S.212)

\subsection{Veränderungen im Alter und Pathologien}

\subsubsection{Presbyopie}

Unter Presbyopie versteht man den stufenweisen, altersbedingten Abfall der Akkommodationsfähigkeit des Auges. Wenn man sich die Vielzahl der Studien zur Akkommodation an Wirbeltieren anschaut, scheinen es tatsächlich nur Primaten zu sein, die diesen systematischen Verlust des Naheinstellungsvermögens im Alter aufweisen. Bei vielen Vogelarten, angefangen bei Tauben bis hin zu Falken, ist es nämlich so, dass sich bei Akkommodation auch die Form ihrer Kornea verändert. Da die Verformbarkeit der Hornhaut bei diesen Tieren im Alter gleich bleibt, werden sie eben auch nicht presbyop. (65, S.169) Beim Menschen hingegen wird das Funktionsvermögen des akkommodativen Apparates wie kaum eine andere physiologische Funktion sehr früh eingeschränkt und nimmt mit dem Alter kontinuierlich ab, was von Duane anhand von Messungen an 4000 Augen bereits 1912 eindrucksvoll gezeigt werden konnte. (44, S.52) Während also ein zehnjähriges Kind noch eine Akkommodationsbreite von $15 \mathrm{dpt}$ besitzt und seinen Nahpunkt bei etwa $7 \mathrm{~cm}$ hat, liegt der Nahpunkt bei einem 40- Jährigen schon in etwa $22 \mathrm{~cm}$ Entfernung und dessen Akkommodationsbreite nur noch bei 4,5 dpt. (86, S.31)

Der Alterungsprozess wirkt sich auf mehrere Komponenten des Akkommodationsapparates aus. Am Ziliarmuskel hat man in histologischen Studien eine kontinuierliche Abnahme der 


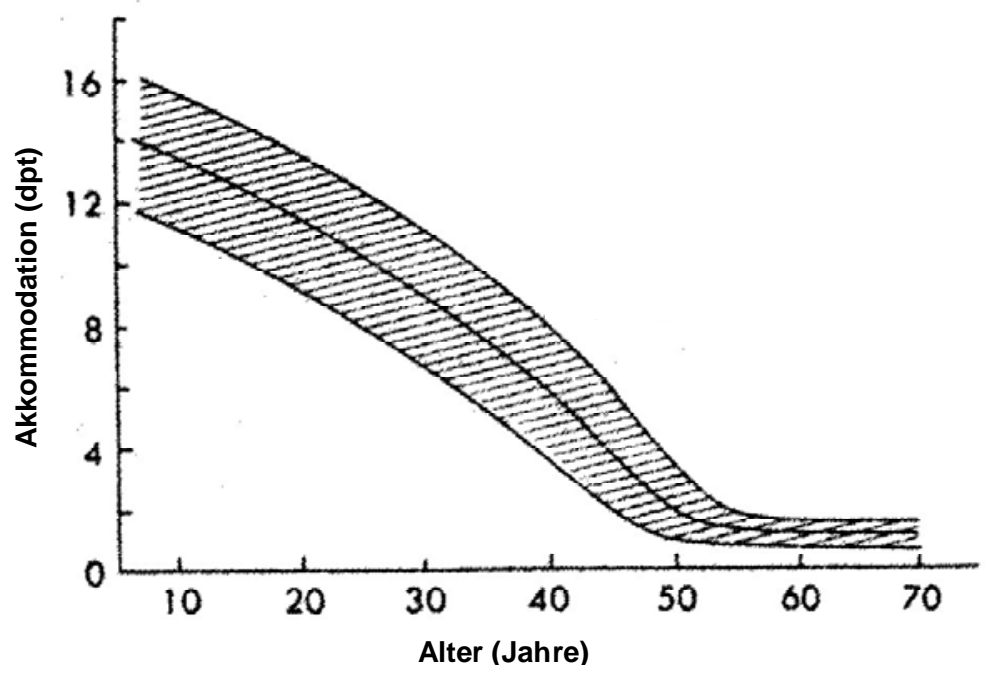

Abbildung 3: Änderung der Akkommodationsbreite mit dem Alter (abgeändert nach 67, S.305)

retikulären und zirkulären Muskelfasern, sowie auch eine Abnahme der Gesamtlänge bei gleichzeitigem Anstieg des Anteils des Bindegewebes beobachtet. (101, S.209) Darüber hinaus verlagert sich der zonulare Ansatz an der Linse im Laufe des Lebens nach vorne. Auch die Linsenkapsel verändert sich mit der Zeit: ihre Dicke, die bei Geburt etwa $11 \mu \mathrm{m}$ beträgt, nimmt bis zum Alter von 60 Jahren zunächst einmal auf die Größe von etwa $20 \mu \mathrm{m}$ zu, um danach wieder leicht abzunehmen. (31, S.220)

Die Linse selbst wächst ein Leben lang. Da die Anzahl von Linsenfaserzellen sowohl am anterioren als auch am posterioren Kortex steigt, nimmt ihre Dicke zu, wobei inzwischen als anerkannt gilt, dass sich ihr äquatorialer Durchmesser nicht altersbedingt ändert. (48, S.1685) Dagegen steigen die Krümmungen der anterioren und posterioren Linsenfläche. (31, S.221) Wenn man nun bedenkt, dass die Linse im Alter stärker gekrümmt ist, überrascht es natürlich, dass gleichzeitig das Naheinstellungsvermögen schwächer wird. Diese Beobachtung, die man das „Linsen-Paradoxon“ nennt, wird zum Teil durch die beschriebenen altersbedingten Veränderungen am Ziliarmuskel begründet (101, S.209), es gibt aber auch mathematische Ansätze, die anhand von Potenzreihen modellieren inwiefern die Brechkraftabnahme der Linse für dieses Phänomen verantwortlich sein kann. (95, S.2111) Da sich das Zentrum der Linsenmasse mit steigendem Alter nach vorne bewegt, kommt es zu einer Abnahme der Vorderkammertiefe. (50, S.265; 25, S.53)

Eine Vielzahl von Studien beschäftigt sich auch mit altersbedingten Veränderungen in der Dynamik der Akkommodation, wobei ihre Ergebnisse nicht immer im Einklang zu stehen scheinen. Während einerseits sowohl bei der fern- zu- nah-, als auch bei der nah- zu- fernAkkommodationszeitspanne eine zeitliche Verlängerung linearen Ausmaßes (etwa 7ms pro Jahr) beobachtet wird (10, S.235), gibt es andererseits auch Studien, die in diesem Zusammenhang keine Abhängigkeit vom Alter messen konnten. (66, S.591) Einig ist man 
sich darüber, dass es große refraktionsbedingte und individuelle Unterschiede bei der Dynamik der Akkommodation zu beachten gilt. (89, S.301; 82, S.159; 91, S.279)

Betrachtet man die aktuellen Erkenntnisse zur Bedeutung der einzelnen Komponenten bei der Entwicklung der Presbyopie stößt man auf eine Vielzahl nicht ganz widerspruchsfreier Theorien.

Eine der Anschauungen macht die Verhärtung der Linse für die Entstehung der Presbyopie verantwortlich. Man spricht hierbei, wie oben bereits erwähnt, von Linsensklerose. Die härtere Linse kann von der Linsenkapsel nicht mehr ausreichend in eine rundere Form gedrückt werden und verbleibt somit in einem fixiert unakkommodierten Zustand. Diese Theorie ist wohl diejenige, die den breitesten Zuspruch hat. (14, S.1544; 36, S.3119)

Andere wiederum sehen in den geometrischen Veränderungen, also dem nach vorne gerückten Ansatz der Zonulafasern und der Verdickung der Linse, die Hauptursache für die Altersweitsichtigkeit. (48, S.1685)

Sehr umstritten sind die von Schachar vorgetragenen Überlegungen zur Presbyopie.

Er erklärt den Akkommodationsmechanismus schon völlig entgegengesetzt zur bereits beschriebenen, anerkannten Theorie von Helmholtz und Gullstrand, indem er bei der Ziliarmuskelkontraktion von einer gleichzeitigen Anspannung der Zonulafasern ausgeht, die den Linsenäquator in Richtung Sklera drückt. Das erfordert natürlich eine andere anatomische und physiologische Auffassung der Akkommodationsvorgänge. So beschreibt er drei verschiedene Zonulafaseranteile, die der vorderen, der äquatorialen und der hinteren Linsenfläche entspringen, auch ihre Ansätze beurteilt er anders. Er postuliert also, dass die Kontraktion des Ziliarmuskels gezielt lediglich die beschriebenen äquatorialen Zonulafasern anspannt, während der Zug an den anderen beiden Fasergruppen reduziert wird. Die damit einhergehende Zunahme des zentralen Linsendurchmessers, die mit einer gleichzeitigen Abflachung der peripheren Linsenfläche auftritt, macht er für die Brechkraftzunahme verantwortlich. (31, S.229) Dementsprechend erklärt er auch Altersweitsichtigkeit mit einer Zunahme des äquatorialen Linsendurchmessers, die es dem Ziliarmuskel unmöglich macht, die Zonulafasern noch ausreichend $\mathrm{zu}$ spannen. Diese Theorie konnte von anderen Studiengruppen jedoch bislang nicht bestätigt werden. (57, S.450)

Schließlich gibt es auch Arbeiten, die eindeutig ablehnen, dass nur einer dieser Aspekte die Erklärung zur Presbyopie liefern kann und ihre Ursache somit als multifaktoriell einstufen. (48, S.569) So beschreibt Robert A. Weale, dass neben den mechanischen Eigenschaften unter anderem auch das veränderte Muster der zellulären Entstehung und Differenzierung 
sowie die pupilläre Myosis und ihre Auswirkungen auf die Akkommodation in diesem Zusammenhang Beachtung finden sollten. (104, S.467)

\subsubsection{Akkommodationsspasmus und Akkommodationslähmung}

Neben den altersbedingten Veränderungen der Akkommodation, gibt es natürlich zahlreiche andere Gründe, die zu Störungen im Akkommodationsvorgang führen können.

Als Akkommodationsspasmus wird eine zwecklose Akkommodationsinnervation bezeichnet, die eine Linsenmyopie zur Folge hat. (86, S.32) Bei der Akkommodationslähmung hingegen handelt es sich meist um Paresen, seltener um Paralysen des Ziliarmuskels. Sie tritt plötzlich oder anfallsweise auf, ein- oder beidseitig, mit und ohne Pupillenbeteiligung und führt dazu, dass sich der Nahpunkt, ähnlich wie bei der Presbyopie, vom Auge entfernt. (86, S.33)

Beide können verschiedene Erkrankungen zur Ursache haben.

Die Therapie richtet sich demnach auch nach dem Grundleiden.

\begin{tabular}{|l|l|}
\hline Akkommodationsspasmus & Akkommodationslähmung \\
\hline - Psychogen (v.a. bei Kindern), Hysterie & - Iridozyklitis \\
- Lange Naharbeit (Spannungsmyopie) & - Glaukom mit Atrophie des Ziliarkörpers \\
- Alkohol & - Isolierte interne Ophthalmoplegie \\
- Medikamente (Cholinergika, Morphine, & - Myotone Dystrophie \\
Digitalis, Sulfonamide, & - Verletzungen, Schädel-Hirn-Trauma \\
- Verletzungen & - Enzephalitis \\
- Morbus Basedow & - Akute idiopathische Polyneuritis \\
- Hirnstammläsionen und Läsionen des N. & - Läsionen parasympathischer Kerngebiete im \\
oculomotorius & Mittelhirn (multiple Sklerose, Syphilis etc.) \\
- Endokrine Ophthalmopathie & - Diabetes mellitus \\
- Diabetes mellitus & - Medikamente (Anticholinergika, Isoniazid, \\
& Antihistaminika, Imipramin u.a.) \\
& - Toxine (Diphtherie, Botulinus, Blei, \\
& Ergotismus) \\
& - Infektionen (Scharlach, Mumps, Masern, \\
& Influenza u.a.) \\
& - Adie-Syndrom (Pupillotonie) \\
\hline
\end{tabular}

Tabelle 1: Differentialdiagnosen bei Akkommodationslähmung und Akkommodationsspasmus, Auswahl nach Augustin (4, S.881) 


\subsection{Stimulation und Messung der Akkommodationsleistung}

Es gibt verschiedene Möglichkeiten, die Akkommodation $\mathrm{zu}$ stimulieren und ihre Auswirkungen am Auge zu erfassen.

Durch pharmakologische Stimulation, anhand von lokal applizierten muskarinischen Agonisten, wie z.B. Pilocarpin, kann man eine unwillkürliche Kontraktion des Ziliarmuskels auslösen. Die Akkommodationsamplitude, die man hierbei mit einem Hartinger Refraktometer misst, scheint zum einen vor allem bei Presbyopen höher zu sein als die, die man mit physiologischer Stimulation erreichen würde. (47, S.795) Zum anderen reagieren Probanden mit hellen Augen stärker auf Pilocarpin als die mit dunklen. (110, S.1883)

Ein Vorteil dieser Methode ist allerdings, dass die Akkommodationsamplitude unabhängig von einem visuellen Reiz oder subjektiven Eindrücken des Probanden erfasst wird.

Eine weitere Möglichkeit wäre, Minus-Gläser vor einem Auge anzubringen, während die Versuchsperson in der Ferne (etwa 6m) Optotypen betrachtet. Dabei wird die konsensuelle Akkommodationsantwort auf dem anderen Auge wiederum mittels eines Hartinger Refraktometers gemessen. Die Stärke der verwendeten Minus- Gläser wird hierbei solange gesteigert bis kein weiterer Anstieg der Akkommodationsantwort zu vermerken ist.

Bei diesen beiden Methoden handelt es sich jeweils um objektive Messungen. (110, S.1878)

In den meisten Studien werden bislang trotzdem subjektive Methoden verwendet.

Sehr beliebt sowohl in klinischer als auch in experimenteller Anwendung ist die sogenannte „push-up“-Technik, bei der die Tafel mit dem Akkommodationsstimulus so lange dem Auge genähert wird bis der Patient den Optotypen nicht mehr scharf erkennen kann. Dabei nimmt man an, dass der reziproke Wert der gemessenen Entfernung der Tafel zum Auge die Akkommodationsamplitude darstellt. (74, S.1435-1436) Diese Methode ist jedoch stark abhängig von der Seh- und Tiefenschärfe des Probanden und neigt überdies dazu, die Akkommodationsamplitude deutlich höher anzugeben, als es objektive Messungen tun. (81, S.247)

Eine weitere mögliche subjektive Messung erfolgt mit Minus-Gläsern, die vor dem Auge angebracht werden. Bestimmend für die Akkommodationsamplitude ist hierbei das stärkste der Versuchsgläser, durch welches das kleinste noch lesbare Sehzeichen nach Snellen vom Probanden noch scharf erkannt werden kann. (31, S.213)

Bei der Betrachtung der verschiedenen Methoden ist es allerdings unverzichtbar, sich vor Augen zu führen, dass Sehen in der Nähe auch durch nicht-akkommodative Mechanismen 
verbessert werden kann. Diese Tatsache macht die Problematik der subjektiven Messmethoden bei der Erfassung der Akkommodationsamplitude mit aus. Sie können nämlich keine Unterscheidung treffen zwischen echter Akkommodation und optischer Kompensation, die z.B. durch multifokale Intraokularlinsen (IOLs) vermittelt werden kann. (74, S.1436)

Dies könnte dementsprechend einen Erklärungsansatz für die Unstimmigkeiten, die durchaus in der Studienlandschaft anzutreffen sind, darstellen.

\subsection{Kritische Würdigung der Literatur und Fragestellung}

\subsubsection{Kritische Würdigung der Literatur}

Insgesamt ist festzustellen, dass die Akkommodation und ihre Auswirkungen Gegenstand zahlreicher Publikationen sind. Die Entwicklung und Reproduzierbarkeit von Methoden zur Stimulation und Erfassung der Akkommodation (112, S.91; 109, S.11; 106, S.207), sowie die Übereinstimmung der Messungen von einzelnen Augenparametern bei Verwendung verschiedener Geräte wurden bereits von einigen Arbeitsgruppen ausführlich beschrieben. (113, S.2325) Auch Veränderungen am Pupillendurchmesser bei der Akkommodation (12, S.154; 43, S.328), sowie der Zusammenhang zwischen der Akkommodationsantwort und verschiedenen Symptomen, wie z.B. Kopfschmerzen oder verschwommener Sehqualität (108, S.175), bzw. Refraktionsanomalien (1, S.105; 69, S.189) wurden schon genauer untersucht. Darüber hinaus ist es bestens bekannt, dass bei der Messung von der Augenlänge Fluktuationen im Tagesverlauf oder in Abhängigkeit von Atmung, sowie Herzfrequenz auftreten können. (102, S.216; 98, S.63) Selbst der Zusammenhang zwischen körperlicher Statur und Augenkomponenten (111, S.1237), sowie der zwischen der Dauer der Naharbeit (92, S.233) oder des Aufmerksamkeitsgrades (58, S.28) und der Akkommodation wurde in kürzlich erschienen Studien hinterfragt.

So scheint es auf den ersten Blick, als wären bei dem Meer an Publikationen zur Akkommodation kaum noch Fragen offen. Dennoch herrscht in vielerlei Hinsicht, wie bereits in vorangegangenen Ausführungen deutlich wird, Uneinigkeit unter den Autoren. So wollen einige Arbeitsgruppen Veränderungen der Augenlänge bei der Akkommodation gemessen haben (94, S.76), während andere dieses kategorisch verneinen. (7, S.523; 28, S.114) 
Schließlich ist die Frage nach der Akkommodationsfähigkeit von Intraokularlinsen wohl diejenige, die im Augenblick wohl die meisten Gemüter bewegt und Bemühungen, eine Lösung für die Presbyopie zu finden, sind groß. (3, S.809; 13, S.1)

\subsubsection{Fragestellung}

Aufgrund der verschiedenen Beobachtungen in der Fachliteratur setzt sich meine Arbeit also zum Ziel die Veränderungen an Augenabschnitten, die tatsächlich während der Akkommodation auftreten, präzise und reproduzierbar zu messen.

Folgende Fragestellungen sollen dabei besonders berücksichtigt werden:

- Vor allem Änderungen der Vorderkammertiefe und die der axialen Augenlänge sollen gemessen und analysiert werden.

- Dabei wird darauf Wert gelegt, dass die Stimulation der Akkommodation ohne pharmakologische Zuhilfenahme erfolgt, so dass die Akkommodationsantwort in physiologischem Rahmen bleibt.

- Darüber hinaus wird besonderes Augenmerk auf eventuelle Unterschiede zwischen isolateraler und konsensueller Akkommodationsantwort gerichtet. In diesem Zusammenhang sollen zwei unterschiedliche Methoden vorgestellt und verglichen werden.

- Dabei soll gewährleistet sein, dass die Ergebnisse der Messungen nicht vom Untersucher abhängen.

- Außerdem befasst sich diese Arbeit mit dem unterschiedlichen Ausmaß der Akkommodationsantwort in Abhängigkeit von verschiedenen Altersgruppen.

- Besonders spannend wird es schließlich sein zu ermitteln, ob sich bei Probanden, die Intraokularlinsen tragen, bei Betrachtung eines Akkommodationsstimulus tatsächlich Veränderungen an Augenabschnitten messen lassen. 


\section{Materialien und Methoden}

\subsection{Materialien}

\subsubsection{IOL-Master}

Die Messungen wurden mit zwei IOL-Master-Geräten gleichen Typs, Softwareversion 3.xx, hergestellt von der Firma Carl Zeiss Meditec, vorgenommen.

Beim IOL-Master handelt es sich um ein kombiniertes Biometriegerät, das in den letzten Jahren vor allem bei der Berechnung der Intraokularlinsen an Bedeutung zugenommen und die bis dahin als Goldstandard geltende Ultraschall-Methode auf diesem Feld weitgehend abgelöst hat. Geschätzt wird an diesem Gerät zunächst einmal, dass es für seine Messungen gar keines direkten Kontaktes zum Patientenauge bedarf, was zum einen für den Probanden angenehmer ist, zum anderen kein Infektionsrisiko in sich birgt. (71, S.85)

Mit dem IOL-Master ist es möglich, Achslänge des Auges, Krümmungsradien der Hornhaut und die Vorderkammertiefe genau auszumessen.

Die Erfassung der Augenlänge erfolgt mittels eines patentierten Verfahrens, der so genannten partiellen Kohärenz-Interferometrie (PCI). Hierbei wird von einem Halbleiter-Diodenlaser infrarotes Licht von $780 \mathrm{~nm}$ Wellenlänge ausgesandt, das im Gerät über einen Strahlenteiler und zwei Spiegel geleitet und letztlich ins Auge geschickt wird. Dort wird es von der Kornea und dem retinalen Pigmentepithel reflektiert. Den Abstand zwischen diesen beiden Flächen gibt er als Achslänge an. (87, S.459) Wie bei der PCI im Allgemeinen, müssen auch beim IOL-Master die gemessenen optischen Strecken in geometrische Längen umgewandelt werden. Als Grundlage verwendet der IOL-Master hierbei das Augenmodell nach Gullstrand. (2, S.283)

Der Messbereich des IOL-Masters umfasst Augenlängen von 14 bis 40 mm. (8, S.86) Eine hohe Genauigkeit und Reproduzierbarkeit der Messungen wurden in zahlreichen Studien nachgewiesen. (37, S.616; 56, S.477; 16, S.235; 20, S.1304) 
Die Keratometrie erfolgt mit Licht von $880 \mathrm{~nm}$ Wellenlänge. Die Hornhautkrümmung wird letzlich anhand des Abstandes zwischen den einzelnen Punktmarken, welche auf die Hornhaut projiziert werden, ermittelt (8, S.11), wobei hier der Messbereich Werte von 5 bis $10 \mathrm{~mm}$ umfasst. $(8, \mathrm{~S} .86)$

Als Vorderkammertiefe wird wie üblich der Abstand zwischen den Schnittbildern der Hornhaut und der Linse angegeben, die durch seitliches Spaltlicht erzeugt werden.

Der Messbereich liegt hier zwischen 1,5 und 6,5 mm. Die Verwendung von Zykloplegika hat keinen verfälschenden Einfluss auf die Genauigkeit der Messung. (35, S.1993; 93, S.27) Auch wenn sie die Genauigkeit um den Faktor fünf steigern soll (20, S.1304), kam eine pharmakologische Einflussnahme in dieser Studie nicht in Frage.

Die Anzeigen aller Messoptionen sind auf zwei Dezimalstellen skaliert.

\subsubsection{Zusatzeinrichtung zur physiologischen Stimulation der Akkommo- dation}

\subsubsection{1 verwendete Materialien}

Für den Bau der Messeinrichtung wurden folgende Materialien verwendet:

- Kompakt-Labornetzgerät 1502D (0-15V, 0-2A) (zwei Stück)

- LED-Digital-Panelmeter-129 (fünf Stück)

- Anzeigenregler

- Spannungsteiler (vier Stück)

- Spezial-Messwiderstände (vier Stück)

- Stufenschalter

- Zwei-Ebenen-Miniaturstufenschalter

- Aluminium-Klammern (drei Stück)

- Schlauchleitung

- Aluminium-Winkelprofil 1m, 3/4 X 3/4 (drei Stück)

- Infusionsständer

- Spiegel $(5 \mathrm{~cm}$ x $3 \mathrm{~cm})$

- Objektträger, transparent ( $5 \mathrm{~cm}$ x 2,5 cm)

- Fixationsarme, dreigelenkig (zwei Stück)

- Spiegel- und Plättchenhalter aus Polypropylen

- Doppelschraubzwingen (zwei Stück) 


\subsubsection{2 experimenteller Aufbau}

In Zusammenarbeit von $\mathrm{PhD}$ Konstantin Kotliar und mir entstanden Überlegungen zum idealen Bau und zur Optimierung der Einrichtung zur Auslösung der Akkommodation. Ihre Anfertigung übernahm $\mathrm{PhD}$ Konstantin Kotliar.

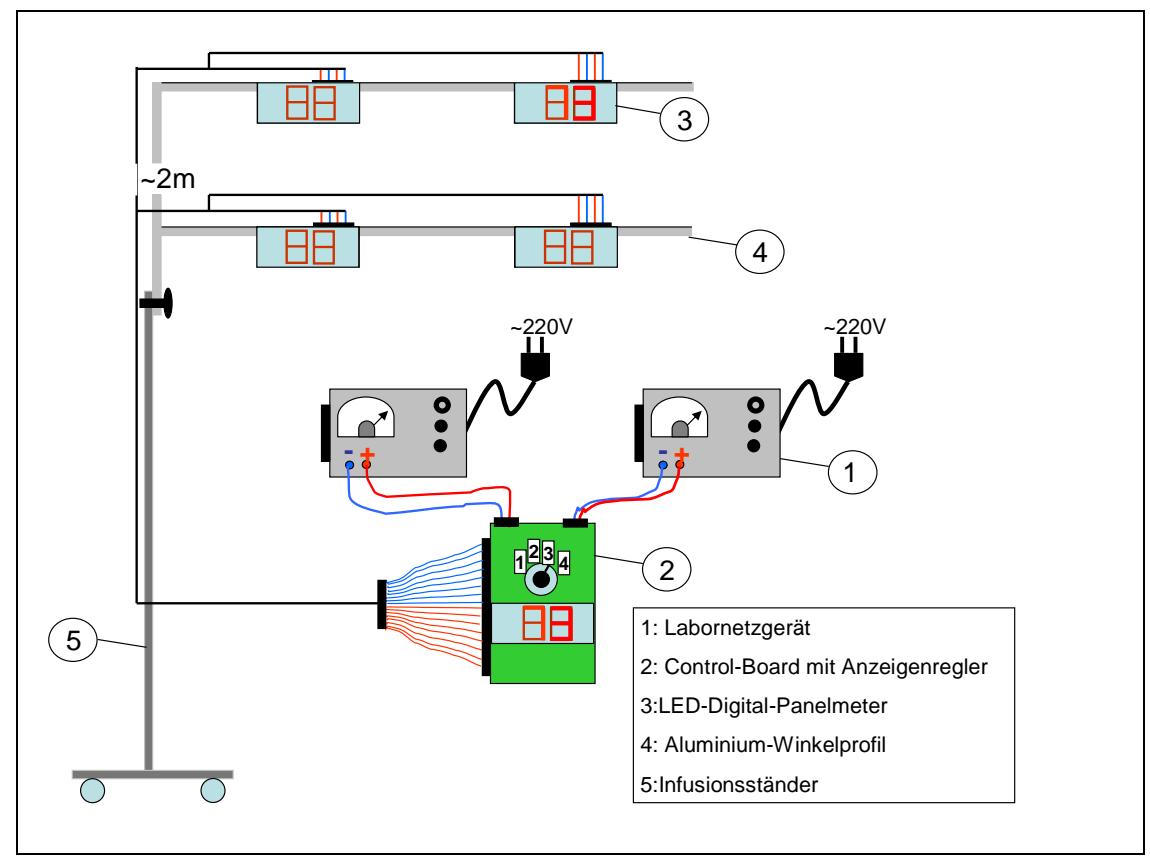

Abbildung 4: Schematische Darstellung der Zusatzeinrichtung zur physiologischen Stimulation der Akkommodation

Am Anfang des Schaltkreises waren die beiden Labornetzgeräte angeordnet. Diese wandelten die $220 \mathrm{~V}$ hohe Spannung, die in sie eingespeist wurde, in regelbare Werte von 0 bis $15 \mathrm{~V}$ um. Eines der beiden Labornetzgeräte hatte zur Aufgabe, die vier Panelmeter mit Strom zu versorgen. Das andere wiederum erzeugte die Spannung, die von den Panelmetern gemessen wurde. Jeweils zwei Panelmeter wiederum wurden an zwei Aluminium- Winkelprofilen angebracht. Ein drittes AluminiumWinkelprofil, das an einem Infusionsständer befestigt wurde, diente zusammen mit

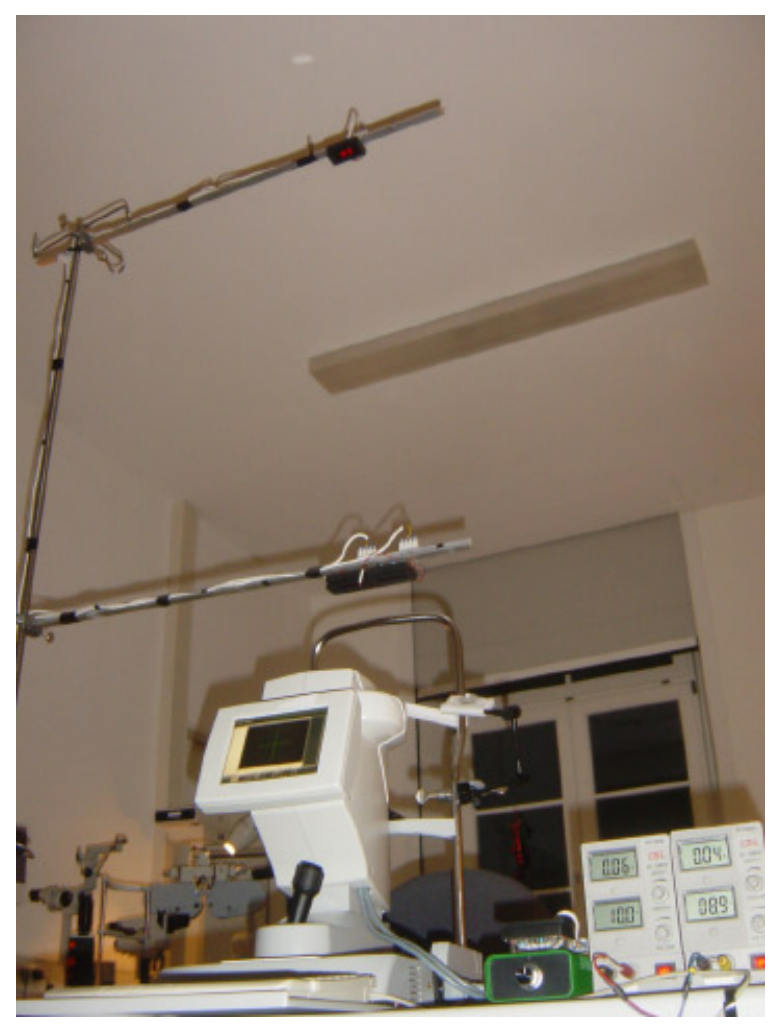

Abbildung 5: Fotografische Darstellung des Versuchsaufbaus 
diesem als Halterung, an der die beiden anderen Stangen in einem Abstand von etwa $2 \mathrm{~m}$ voneinander stabil montiert wurden. Diese mobile Konstruktion konnte gut neben dem IOLMaster positioniert werden. Die Höhe der Panelmeter wurde hierbei stets so angepasst, dass die Abstände für alle Probanden die gleichen waren.

Die vier Panelmeter waren identischen Aussehens. Sie zeigten die Spannung an, die sie vom Netzgerät empfingen. Dabei konnten sie Zahlen von 00 bis 99 präsentieren.

Dem Untersucher war es durch an den Netzgeräten angebrachte Regler also möglich, zum einen die Anzeige an den Panelmetern zu verändern, zum anderen die Leuchtstärke ihrer Ziffern zu regulieren. Darüber hinaus befand sich vor dem Untersucher ein Control-Board, das den Labornetzgeräten und den Panelmetern zwischengeschaltet war. Mit diesem konnte er genau einstellen, an welchem der vier Panelmeter die zwei Zufallsziffern rot aufleuchten sollten. Die restlichen drei LED-Anzeigen blieben in dieser Zeit schwarz, so dass die Probanden nicht durch andere leuchtenden Zahlen abgelenkt werden konnten.

Dem Untersucher wurde zur gleichen Zeit auf dem Control-Board die Zahl angezeigt, die dem Probanden aktuell präsentiert wurde. So konnte er mühelos überprüfen, ob der Proband die Ziffern richtig erkennt und vorliest.

\subsubsection{Probanden und Ausschlusskriterien}

\subsubsection{Probanden}

Es sollten vier verschiedene Gruppen an der Studie teilnehmen: Kinder, Jugendliche, Erwachsene und pseudophake Erwachsene. In jeder Gruppe wurden 15 Probanden beider Geschlechter gemessen, insgesamt also 60 Personen.

\subsubsection{Ausschlusskriterien}

Für die drei gesunden Probandengruppen, also Kinder, Jugendliche und Erwachsene, galten folgende Ausschlusskriterien:

- Refraktionsanomalie von mehr als 1,8 dpt

- Astigmatismus von mehr als $0,5 \mathrm{dpt}$

- Anisometropie von mehr als 1,0 dpt

- Vorangegangener chirurgischer Eingriff am Auge

- Entzündungen oder andere Erkrankungen am Auge

- Amblyopie 
- Strabismus

- Vorangegangene Applikation von Medikamenten am Auge

Die Gruppe der pseudophaken Erwachsenen hatte ja bereits einen oder mehr chirurgische Eingriffe am Auge hinter sich.

\subsection{Methoden}

\subsubsection{Anamnese und Aufklärung}

\subsubsection{Anamnese}

Vor Studienbeginn wurde bei jedem Patienten eine ausführliche standardisierte Eigen-, bzw. bei Kindern eine Fremdanamnese erhoben.

Die Angaben zum Visus wurden im Fall von Erwachsenen und Pseudophaken ihrer Patientenkarteikarte entnommen. Diese beiden Gruppen wurden unmittelbar vor oder nach der Messung ausnahmslos augenärztlich untersucht.

Bei Kindern und Jugendlichen galten die Angaben, die die Probanden gemacht haben. Es wurde hierbei darauf geachtet, dass die Untersuchungen, auf die sich die Probanden bezogen, nicht länger als zwei Jahre zurücklagen.

\subsubsection{Aufklärung}

Allen Probanden wurde zunächst einmal die Fragestellung der Studie in einem Gespräch dargelegt. Sie wurden darüber hinaus ausführlich über das Funktionsprinzip des IOL-Masters informiert und es wurde ihnen verdeutlicht, dass bei den Messungen photochemische Strahlen auf die Netzhaut treffen. Sie wurden darüber aufgeklärt, dass der Grenzwert für Achslängenmessungen bei 20 Messungen pro Auge und Tag liegt, und dass bei Einhaltung dieser Bestimmung nach Angaben des Herstellers die Gefahr der Schädigung durch optische Strahlung äußerst gering ist und die photometrischen Werte des IOL-Masters deutlich unterhalb der als hoch einzustufenden Gefährdungspotentiale liegen.

Alle Probanden zeigten sich nach ausführlicher Aufklärung mit der Durchführung der Messungen einverstanden. Bei Kindern wurde die Einverständniserklärung der Eltern eingeholt. 


\subsubsection{Grundlegende Messungen mit dem IOL-Master}

Noch vor Beginn der Messungen wurden zunächst die Daten des Probanden, also Nachname, Vorname und Geburtsdatum eingegeben. Außerdem war es notwendig, das Gerät auf den zu Untersuchenden einzurichten. Nach der richtigen Einstellung der Kinnstütze wurde der Patient aufgefordert auf das gelbe Fixierlicht zu achten. Auf dem Untersucherbildschirm bei dieser Einstellung erschienene Fadenkreuz wurde in Pupillenmitte platziert, die sechs Lichtpunkte wurden scharf gestellt und zum Fadenkreuz zentriert.

\subsubsection{Messung der Augenlänge}

Nun wurde zum Modus der Augenlängenmessung gewechselt.

Auf dem Bildschirm erschien an dieser Stelle ein deutlich kleinerer Ausschnitt des Auges, es war wiederum ein Fadenkreuz mit einem Kreis in der Mitte zu sehen.

Der Patient wurde aufgefordert auf das hier rote Fixierlicht zu schauen.

Anschließend erfolgte nach einer Scharfstellung des Justierlichtreflexes die erste Messung. Die Augenlänge wurde jeweils vier Mal gemessen und die Werte wurden dokumentiert.

Auf dem Display des IOL-Masters erschien nach jeder Messung ein Graph, der das Videobild überlagerte. Dieser zeigte bei ordnungsgemäßer Durchführung und ruhiger Haltung des Probanden einen Hauptpeak an, der von der Reflektion des Messlichtes vom retinalen Pigmentepithel herrührte. Außerdem entstanden stets auch symmetrisch angeordnete Nebenmaxima, die jedoch durch die Lichtquelle bedingt waren. Neben dem Graphen wurde die gemessene Länge und ein SNR (signal-to-noise-ratio)-Wert, Maß für das Signal-RauschVerhältnis, angezeigt.

SNR-Werte die zwischen 1,6 und 1,9 lagen, wurden schon vom Gerät als unsicher bewertet. Es wurde bei den Messungen darauf geachtet, dass der SNR-Wert im höheren Bereich, möglichst über 7,0 lag.

\subsubsection{Keratometrie}

Anschließend wurde in den Modus der Keratometrie gewechselt.

Der Proband wurde aufgefordert, zunächst einmal zu zwinkern, dann die Augen weit offen zu lassen und auf das gelbe Fixierlicht zu schauen. Auf dem Display erschienen bei dieser Einstellung zwei Kreise und sechs Punkte. Bei den Messungen wurde besonders Wert darauf gelegt, dass keine der Messmarken von den Augenlidern verdeckt wurde, und dass diese optimal scharf eingestellt waren, sowie zwischen den beiden Hilfskreisen lagen, symmetrisch zum Fadenkreuz. 
Bei Auslösung der Messung mit dem Joystick führte das Gerät innerhalb kurzer Zeit fünf Messungen durch. Danach zeigte der IOL-Master die Radien bzw. die Brechkräfte der beiden Hauptschnitte und die entsprechenden Achslagen an. (8, S.30)

\subsubsection{Messung der Vorderkammertiefe}

Der IOL-Master gibt als Vorderkammertiefe (VKT) den Abstand des vorderen Hornhautscheitels vom vorderen Linsenscheitel an. Dieser enthält also die Hornhautdicke und bedarf daher der Kenntnis des Hornhautradius. Deshalb ist es notwendig die Keratometrie durchzuführen bevor man in den VKT-Modus wechselt.

In dieser Einstellung schaltete sich automatisch das Spaltlicht mit ein. Der Proband wurde aber noch einmal darauf aufmerksam gemacht, trotzdem auf das gelbe Fixierlicht zu schauen. Auf dem Bildschirm erschien bei dieser Einstellung ein Schnittbild der Hornhaut und der Linse, sowie ein Rechteck und ein Fixierpunkt.

Bei den Messungen wurde vor allem darauf geachtet, dass der Fixierpunkt scharf zu sehen war, optimal im Rechteck lag und sich zwischen dem Hornhaut- und dem Linsenschnittbild, nahe an der Linse, befand. Außerdem wurde das Gerät so eingestellt, dass das systembedingt unscharfe Hornhautbild frei von Reflexen war, und das Bild der Linsenvorderkante in der Pupille zu sehen war.

Während der Messung flackerte der Spaltprojektor. Bei Ertönen des Signaltons war die Messung beendet und das Spaltlicht leuchtete kontinuierlich. Da die Auswertung anschließend ungefähr noch $15 \mathrm{~s}$ dauerte, wurde der Proband an dieser Stelle gebeten sich zurückzulehnen. So konnte er das Ergebnis abwarten ohne vom Spaltlicht geblendet zu werden.

\subsubsection{Sondereinstellungen und Korrekturen}

\subsection{Sondereinstellungen}

Vor der Messung pseudophaker Erwachsener war es unabdingbar, der Tatsache, dass sie eine Kunstlinse im Auge haben, entsprechend Rechnung zu tragen. Unter dem Modus der Augenlängenmessung wurde über das Menü „AL Zusatzfkt.“ das Linsenmaterial eingestellt. Erst dann konnte die Messung der Augenlänge in dieser Gruppe korrekt durchgeführt werden.

\subsection{Korrekturen}

Bei zwei Probanden aus der Gruppe der pseudophaken Erwachsenen war es notwendig die Ergebnisse der Augenlängenmessung einer manuellen Korrektur zu unterziehen. Es traten bei diesen Probanden zwei hohe Ausschläge auf, wobei der erste höhere Peak von der 
Intraokularlinse herrührte und erst der zweite Peak von der Netzhaut kam. In diesen Fällen wurde zunächst einmal das Bild des Graphen vergrößert. Anschließend wurde der Pfeilkursor auf den weißen Punkt, der das bisherige Maximum anzeigte, gebracht. Mit Hilfe der Maus wurde nun der hintere Peak, der von der Netzhaut kam, markiert. Der Messkursor rastete automatisch bei diesem ein und die richtige Augenlänge, sowie der zugehörige SNR-Wert erschienen auf dem Bildschirm.

\subsubsection{Messungen mit Plättchen und Spiegel}

\subsubsection{Versuchsaufbau Plättchen}

Zweck dieses Versuchsaufbaus war es, das Auge, das ausgemessen wurde, zur Akkommodation zu bringen und dabei zu vermessen.

Seitlich an dem Gerüst des IOL-Masters wurden rechts und links mittels Doppelschraubzwingen zwei Fixationsarme angebracht. Da diese dreigelenkig waren, konnte man sie leicht bewegen und in die richtige Position bringen. Am distalen Teil der Fixationsarme war jeweils ein Plättchenhalter aus Polypropylen positioniert, der zuvor genau ausgemessen und zugeschnitten wurde, so dass er dem anschließend in ihn eingebrachten Objektträger stabilen Halt bot.

Der Proband konnte anhand der Fixationsarme das halbdurchlässige Glasplättchen optimal vor dem Auge einstellen. Durch Drehen an dem Plättchenhalter war es möglich, den Winkel des Plättchens so einzustellen, dass dieses den Akkommodationsreiz aus der vertikalen Richtung in die horizontale und somit in die Sehachse des Betrachters lenkte.

Über beiden Augen waren ja in Abständen von etwa $16 \mathrm{~cm}$ und $216 \mathrm{~cm}$ die Panelmeter aufgebaut, die Zahlen anzeigen konnten. Als eine der Anzeigen eingeschaltet wurde, konnte der Proband bei entsprechender Einstellung des Plättchens in diesem das Spiegelbild der beiden Ziffern erkennen. Die Einspiegelung sollte so erfolgen, dass sich das Fixierlicht des IOL-Masters etwa in der Mitte zwischen den beiden Ziffern befindet. Das war besonders wichtig, denn der Proband war ja aufgefordert, auf die Zahlen zu achten. Dennoch musste sichergestellt werden, dass die Augenachse mit dem Fixierpunkt möglichst auf einer Linie liegt, um Präzision bei den Messungen zu gewährleisten.

Die richtige Einstellung des Plättchens erforderte anfangs einige Übung. Sobald der Proband jedoch ein wenig Gefühl für die Justierung der Fixationsarme und des Plättchenhalters entwickelte, konnte er diese optimal und sehr stabil positionieren. 
Die Versuchspersonen wurden gefragt, ob sie die Zahlen scharf erkennen können und gebeten, diese vorzulesen. In diesem Augenblick wurden die Messungen vorgenommen.

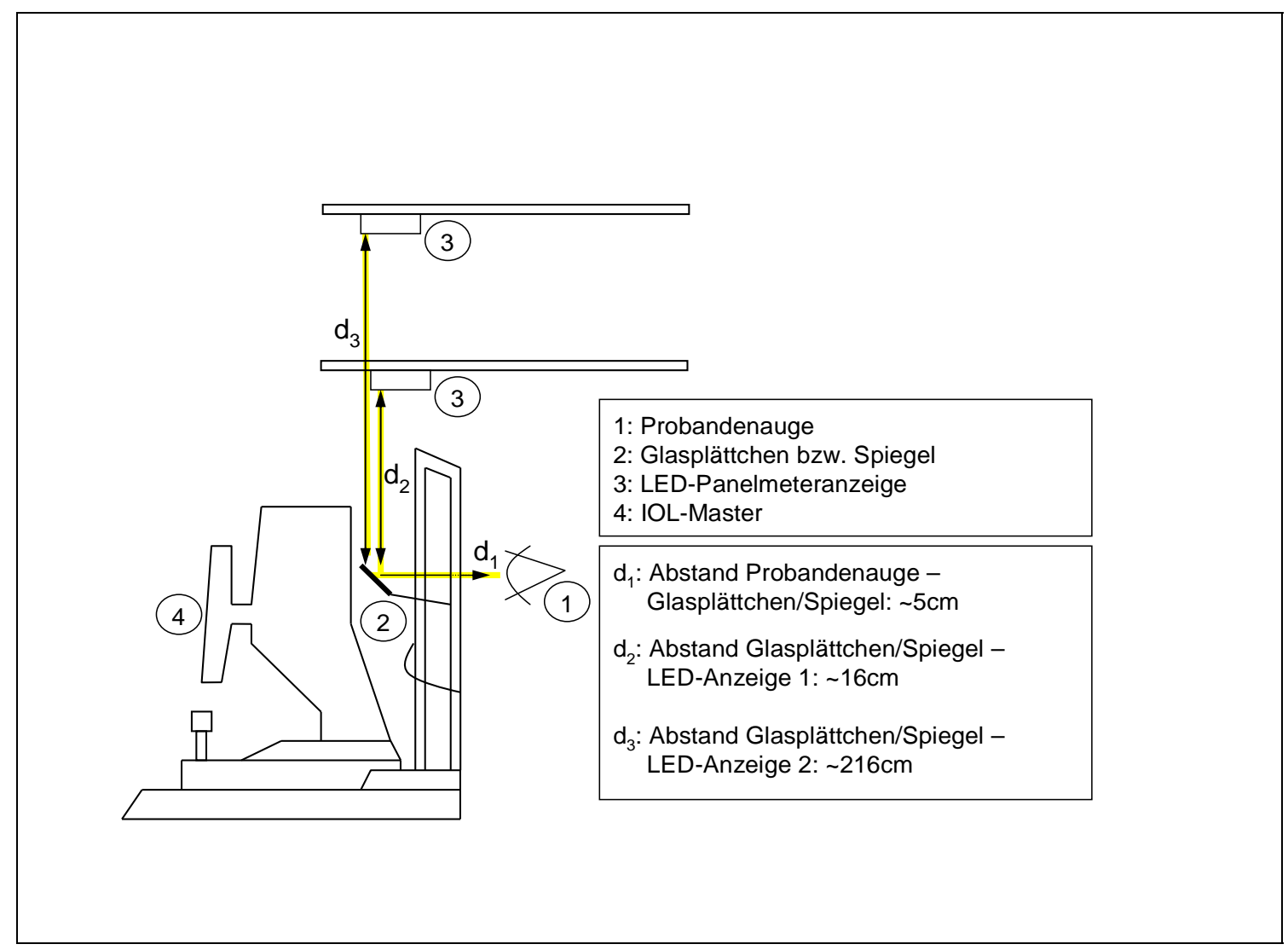

Abbildung 6: schematische Darstellung der Versuchsanordnung

\subsubsection{Versuchsaufbau Spiegel}

Etwas anders war die Situation bei den Messungen mit dem Spiegel. Ziel war es ein Auge auszumessen, während das kontralaterale Auge zur Akkommodation gebracht wird. Es sollte also die konsensuelle Akkommodationsantwort erfasst werden.

Diese Versuchsanordnung wurde im Laufe der Zeit optimiert. Zuerst war sie so gedacht, dass die Probanden den Spiegel in der Hand halten, was sich jedoch vor allem bei älteren Probanden als nicht $\mathrm{zu}$ stabil erwies. Daraufhin wurde ein Spiegel auf einem Polypropylenhalter befestigt. Dieser wiederum wurde, analog zum Plättchenhalter an den Fixationsarmen angebracht. Diese Anordnung erwies sich nicht nur als deutlich störungsfreier, sondern auch als praktischer, denn der Proband konnte sich bei den Messungen der Vorderkammertiefe zwischendurch zurücklehnen. Dabei blieb ja der Spiegel optimal eingestellt, so dass er ihn nicht immer wieder neu vor dem Auge platzieren musste.

Bei allen hier vorgestellten Versuchspersonen wurde ein Spiegelhalter benutzt.

Dabei erfolgte die Messung folgendermaßen: 
Wenn also die Akkommodationsantwort am rechten Auge gemessen werden sollte, kamen die beiden Panelmeter über dem linken Auge zum Einsatz. Der Proband stellte sich die Einspiegelung der Ziffern vor dem linken Auge so ein, dass er mit dem rechten Auge gerade auf den Fixationspunkt blickte. Auf diese Weise konnte er sich auf das Ablesen der Zahlen konzentrieren und lag trotzdem mit der Augenachse der zu messenden Seite auf einer Linie mit der Fixation des IOL-Masters.

Bei Messungen am linken Auge, wurde die Akkommodation entsprechend rechts stimuliert. Auch hier wurde bei allen Probanden evaluiert ob die Ziffern scharf zu erkennen sind, und stets auch überprüft, ob tatsächlich die richtigen Zahlen vorgelesen wurden.

\subsubsection{Messungen mit zwei Untersuchern}

\subsubsection{Planung}

Um zu überprüfen ob die Ergebnisse der Messungen vom Untersucher abhängen, wurden fünf jugendliche Probanden nicht nur von mir, sondern zusätzlich noch von einer erfahrenen Augenschwester gemessen.

Es sollte sowohl die Reihenfolge der Probanden als auch die der Messungen randomisiert werden. Dies erfolgte, indem die teilnehmenden Personen zunächst Zettel zogen, mit denen ihre Reihenfolge beim Untersucher festgelegt wurde, was für beide Untersucher getrennt vorgenommen wurde. Anschließend wurde mit dem gleichen Prinzip für jeden Probanden festgelegt, ob zuerst die Messung am nahakkommodierten oder am unstimulierten Auge erfolgen sollte. Diese wurden getrennt voneinander durchgeführt. Dabei wurden die Ergebnisse der einzelnen Messreihen erst einmal auf getrennten Zetteln aufnotiert und erst zum Schluss zusammengeführt.

\subsubsection{Durchführung}

Folgende Messungen wurden von beiden Untersuchern jeweils an beiden Augen durchgeführt:

- Augenlänge ohne Akkommodation

- Hornhautkrümmung

- Vorderkammertiefe ohne Akkommodation

- Augenlänge mit Nahakkommodation, Methode: Plättchen

- Augenlänge bei Nahakkommodation, Methode: Spiegel

- Vorderkammertiefe bei Nahakkommodation, Methode: Plättchen 
- Vorderkammertiefe bei Nahakkommodation, Methode: Spiegel

Die Messungen der Augenlängen und der Vorderkammertiefen erfolgten in jedem der gefragten Messgänge jeweils zweimal.

\subsubsection{Versuchsablauf}

Grundsätzlich ist zu betonen, dass die Planung und Durchführung dieser Arbeit unter ausdrücklicher Befolgung der ethischen Grundsätze des Weltärztebundes nach der Deklaration von Helsinki geschahen.

Nach einer ausführlichen Anamnese, Aufklärung und der Einholung einer Einverständniserklärung konnte der Proband Platz nehmen. Zunächst wurde die Stuhlhöhe und die Kopfstütze des IOL-Masters auf ihn eingestellt, anschließend konnte die Höhe der LED-Digitalanzeige jedem einzelnen angepasst werden.

Erst einmal wurden beide Augen ausgemessen, ohne dass ihnen ein Akkommodationsreiz präsentiert wurde. Dies erfolgte beidseitig und in der üblichen Reihenfolge: Augenlänge, Hornhautkrümmung, Vorderkammertiefe.

Bei den Probanden der Gruppen Jugendliche, Erwachsene und pseudophake Erwachsene wurden sowohl die Messungen der Augenlänge als auch jene der Vorderkammertiefe in allen hier beschriebenen Messreihen jeweils viermal durchgeführt. Bei Kindern wurde die Anzahl auf jeweils zwei Messungen beschränkt, da danach die Konzentration der Kinder stark nachließ.

Nach der Erfassung der Grundmaße der Augen, wurde die Akkommodation mittels Plättchen stimuliert. An dieser Stelle wurde den Probanden zunächst der fernere Reiz, also das Panelmeter in etwa 2 m Höhe präsentiert. Sie wurden erneut darauf hingewiesen, dass sie die Zahl spiegelverkehrt sehen würden. Als sie sich die Ziffern im Plättchen scharf einstellten und diese ablasen, wurden die Messungen der Augenlänge und der Vorderkammertiefe wiederholt. Anschließend wurden die Ziffern des nahen LED-Digitalanzeige im Plättchen eingespiegelt, die Probanden konzentrierten sich auf diese und Messungen zur Nahakkommodationsantwort wurden durchgeführt.

Nachdem die Prozedur mit dem Plättchen auf beiden Augen wiederholt wurde, ob rechtes oder linkes Auge zuerst spielte keine Rolle, konnte zu den Messungen mit dem Spiegel gewechselt werden.

Auch hier wurde zunächst die Fernakkommodation und dann die Naheinstellung stimuliert. Augenlänge und Vorderkammertiefe wurden vermessen und dokumentiert. 


\subsubsection{Dokumentation}

Alle Messergebnisse wurden auf einem standardisierten Bogen notiert. (s. Appendix I) Die Ergebnisse der Messungen mit zwei Untersuchern wurden nach Abschluss der Untersuchungen auf einem standardisierten Blatt zusammengeführt. (s. Appendix I)

\subsubsection{Datenanalyse und Statistik}

Datenanalyse und Statistik wurden EDV-gestützt unter Anwendung der Programme „Microsoft Excel für Windows Office XP“ und „SPSS“, Version 13.0 durchgeführt. Für die Erstellung der Bland-Altman-Diagramme wurde die statistische Software „Medcalc“ verwendet.

Die deskriptive statistische Auswertung erfolgte entsprechend dem Datenniveau:

Bei quantitativen Größen wurden Mittelwert, Standardabweichung, sowie ausgesuchte Quantile (Minimum, Median, Maximum) ermittelt. Qualitative Merkmale wurden anhand ihrer Häufigkeitsverteilung beschrieben.

Die Ergebnisse wurden auf Signifikanz getestet. Für die Überschreitungswahrscheinlichkeit $p$ galten folgende Signifikanzunterschiede: $p \leq 0,05$ bedeutete einen signifikanten Unterschied, bei $p>0,05$ wurde von einer nicht-signifikanten Beobachtung ausgegangen.

Für die Veränderungen innerhalb eines Augenmerkmals, also Vorderkammertiefe oder Augenlänge, wurde ein zweiseitiger, gepaarter t- Test verwendet.

Bei Mehrfachvergleichen zwischen den Gruppen wurde der Vielzahl der Vergleiche durch die oneway-ANOVA-Analyse mit dem post-hoc-Bonferroni-Test Rechnung getragen.

Zur Prüfung der bivariaten Korrelation zwischen den beiden Methoden und zwischen den Messungen der beiden Untersucher wurde der Rangkorrelationskoeffizient von Pearson verwendet. Hier galt ein zweiseitiges Signifikanzniveau von 0,01 . 


\section{Ergebnisse}

An dieser Stelle sollen die Ergebnisse der Messungen vorgestellt werden.

Bei den Graphen - sofern sie der Abbildung von Augenparametern und ihren Veränderungen dienen - wird aus Übersichtlichkeitsgründen bei den gesunden drei Gruppen jeweils nur das rechte Auge berücksichtigt, bei pseudophaken Erwachsenen ist es entsprechend das Auge mit der Kunstlinse.

\subsection{Beschreibung des Probandenkollektivs}

An den Untersuchungen nahmen 60 Probanden (34 männliche, 26 weibliche) teil. Einige von ihnen wurden aus dem Freundeskreis rekrutiert, die Mehrheit jedoch waren Patienten des Augen-Medizinischen Versorgungszentrums Dr. Kölbl, Prof. Dr. Sachsenweger und Kollegen in Landshut. Die Messungen wurden teils in der Augenklinik und Poliklinik des Klinikum rechts der Isar, teils im Augen-Medizinischen Versorgungszenrum Dr. Kölbl, Prof. Dr. Sachsenweger und Kollegen durchgeführt.

Das Alter der Probanden lag zwischen sieben und 70 Jahren. Im Durchschnitt waren die Kinder elf und die Jugendlichen 22 Jahre alt. Etwa vergleichbar gestaltete sich die

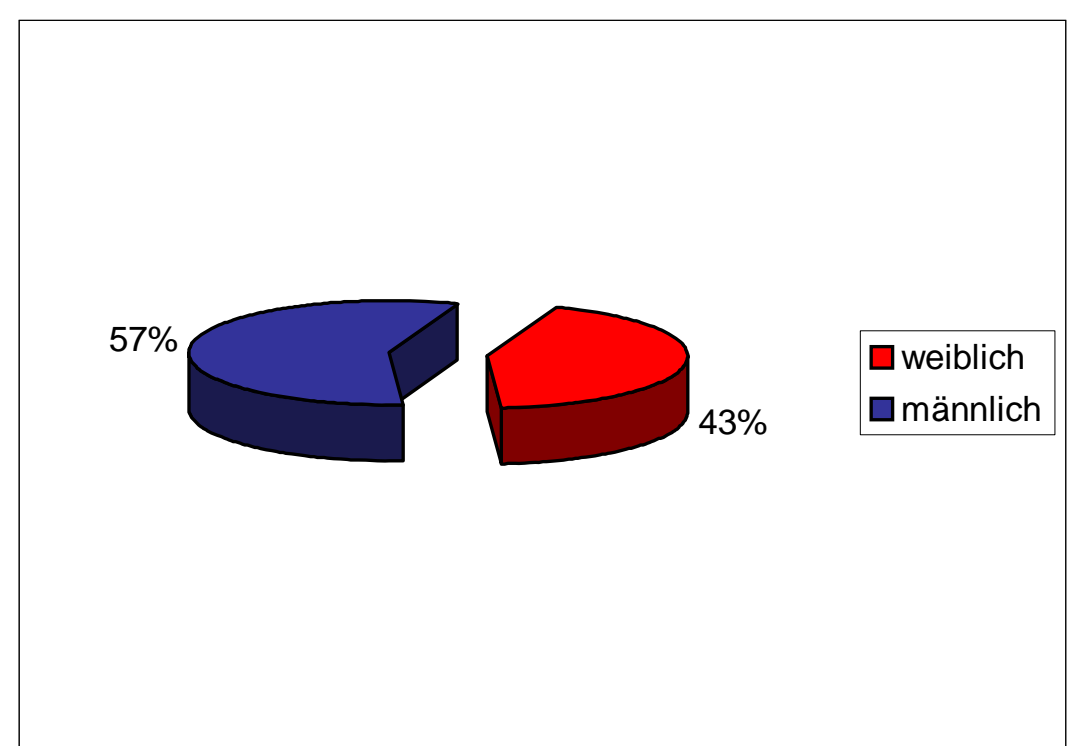

Abbildung 7: Geschlechterverteilung im Probandenkollektiv von 56, bzw. 60 Jahren. Die deskriptive Statistik mit demographischen Angaben ist in Tabelle 2 dargestellt. Die Refraktionsanomalien reichten von $-1,5$ bis 1,5 dpt bei Kindern, von -1 bis 0,5 dpt bei Jugendlichen und von $-1,8$ bis $1,8 \mathrm{dpt}$ bei Erwachsenen und Pseudophaken. 40\% $(\mathrm{N}=6)$ der 
Pseudophaken hatten in beiden Augen eine Kunstlinse, jedoch wurde auch bei ihnen für die statistische Auswertung nur ein Auge berücksichtigt.

\begin{tabular}{|l||l|l|l|l|}
\hline & Kinder & Jugendliche & Erwachsene & Pseudophake \\
\hline MW & 10,5 & 21,9 & 55,7 & 59,9 \\
\hline Median & 11,0 & 23,0 & 52,0 & 63,0 \\
\hline Min & 7,0 & 17,0 & 48,0 & 48,0 \\
\hline Max & 14,0 & 24,0 & 69,0 & 70,0 \\
\hline SD & 1,9 & 2,1 & 7,3 & 7,8 \\
\hline
\end{tabular}

Tabelle 2: Übersicht über Altersverteilung im Probandenkollektiv

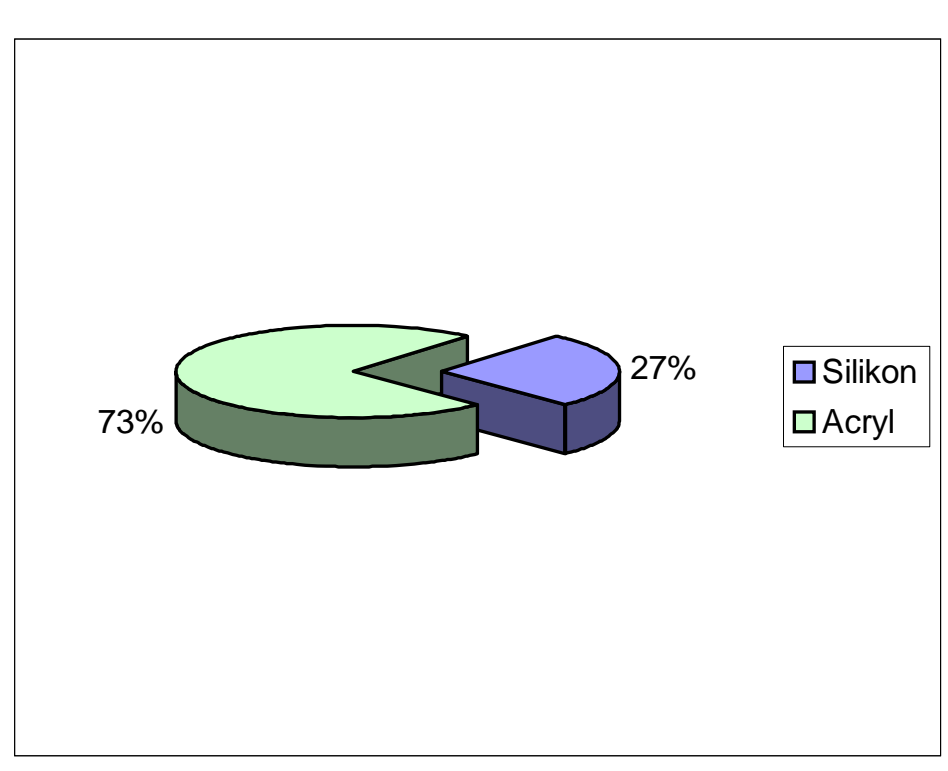

Abbildung 8: Verteilung der Kunstlinsen
Während bei $27 \% \quad(\mathrm{~N}=4)$ der Pseudophaken eine Silikonlinse implantiert worden war, fand man bei $73 \%(\mathrm{~N}=11)$ eine Acryllinse vor. Nach anamnestischer Angabe war bei allen katarakt-operierten Patienten der Eingriff gut verlaufen. Ein Nachstar oder andere operationsbedingte Komplikationen waren nicht aufgetreten.

\begin{tabular}{||l||r|r|r|}
\hline \multicolumn{1}{||l||}{ Linsentyp } & Silikon & \multicolumn{2}{c|}{ Acryl } \\
& & hydrophob & hydrophil \\
\hline $\begin{array}{l}\text { Anzahl der } \\
\text { Träger }\end{array}$ & 4 & 4 & 7 \\
\hline
\end{tabular}

Tabelle 3: Übersicht Linsen bei pseudophaken Probanden 


\subsection{Ergebnisse innerhalb der einzelnen Gruppen}

Zunächst einmal lohnt es sich, einen Blick auf die Verteilung der Parameter AL und VKT innerhalb der vier Gruppen zu werfen. Dabei werden erst die Werte besprochen, die ohne Akkommodationsstimulus mit dem IOL-Master gemessen wurden. Ihre Mittelwerte, deren Extreme und Standardabweichungen werden im Folgenden vorgestellt.

\subsubsection{Kinder}

In der Gruppe der Kinder wurden, im Gegensatz zu den anderen drei Gruppen, alle Parameter lediglich zweimal gemessen. Dies geschah vor allem aus dem Grund, dass die Messungen so erheblich schneller durchgeführt werden konnten und die Kinder in dieser kürzeren Zeitspanne deutlich besser in der Lage waren, ihre Konzentration auf einem hohen Niveau zu halten.

\subsubsection{Vorderkammertiefe}

Bei Kindern belief sich die Vorderkammertiefe im Bereich von 3,30 bis 4,01 mm auf dem linken und von 3,33 bis 3,98 $\mathrm{mm}$ auf dem rechten Auge, wobei die Mittelwerte der VKT der beiden Augen kaum differierten. Die deskriptive Statistik zur VKT bei Kindern wird in Tabelle 4 dargelegt.

\begin{tabular}{|l||l|l|}
\hline & rechtes Auge & linkes Auge \\
\hline MW & 3,62 & 3,64 \\
\hline Median & 3,62 & 3,61 \\
\hline Min & 3,33 & 3,30 \\
\hline Max & 3,98 & 4,01 \\
\hline SD & 0,19 & 0,19 \\
\hline
\end{tabular}

Tabelle 4: Übersicht über VKT bei Kindern, Angaben in mm

Die in der Tabelle 4 vorgestellten Minima, Maxima und Standardabweichungen beziehen sich, wie auch in allen folgenden Tabellen auf die Mittelwerte der Einzelmessungen der Probanden.

Anhand des Histogramms in Abbildung 9 wird die Verteilung der VKT der rechten Augen in der Kindergruppe verdeutlicht. Die Streuung der intraindividuellen Einzelwerte lag hier in $53 \%(\mathrm{~N}=8)$ der Fälle bei 0,01 mm. Bei 13\% (N=2) der kleinen Probanden wurden jedoch 


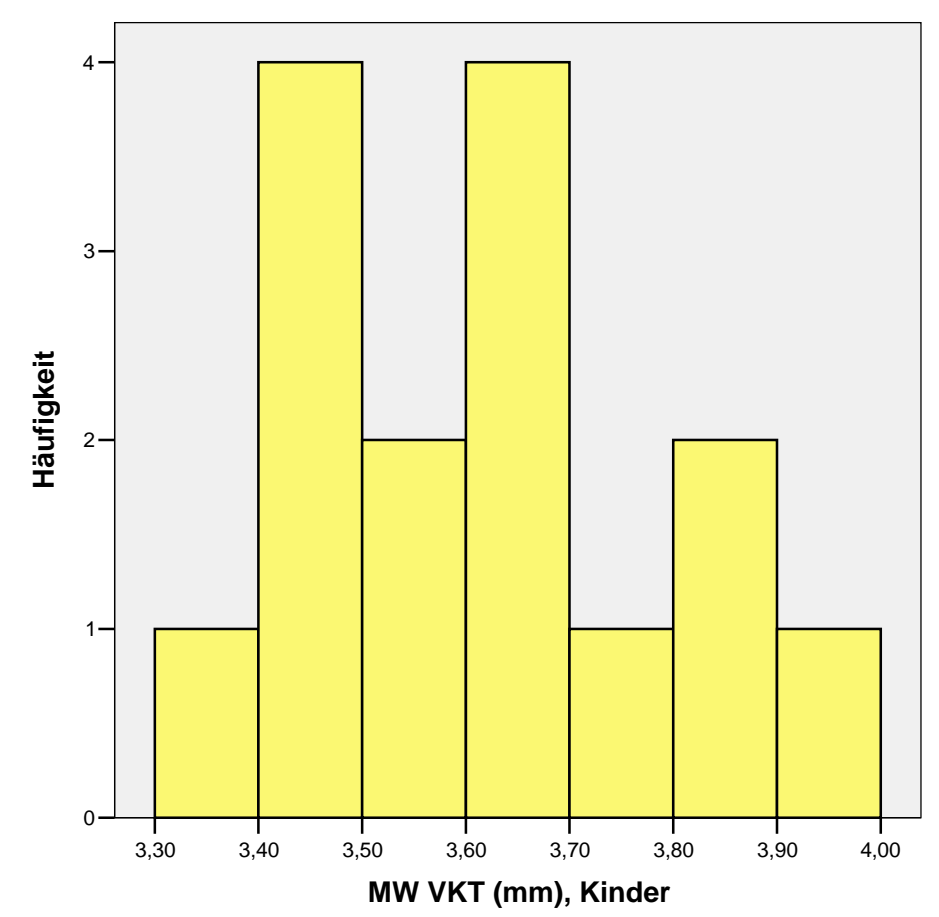

Abbildung 9: Histogramm zur Verteilung der VKT bei Kindern

auch größere Standardabweichungen von 0,04 mm beobachtet. Die Korrelation der VKT zwischen den beiden Augen lag bei r=0,97.

\subsubsection{Augenlänge}

Bei der AL wurden in der Kindergruppe Werte von 22,45 bis $24,35 \mathrm{~mm}$ auf dem rechten und von 22,50 bis 24,29 mm auf dem linken Auge gemessen. Auch hier lagen die Mittelwerte eng beieinander: 23,19 $\mathrm{mm}$ auf dem rechten und 23,16 $\mathrm{mm}$ auf dem linken Auge.

\begin{tabular}{|l||l|l|}
\hline & rechtes Auge & linkes Auge \\
\hline MW & 23,19 & 23,16 \\
Median & 23,29 & 23,11 \\
Min & 22,45 & 22,50 \\
Max & 24,35 & 24,29 \\
SD & 0,49 & 0,49 \\
\hline
\end{tabular}

Tabelle 5: Übersicht über AL bei Kindern, Angaben in mm

$73 \%$ der AL-Werte befanden sich bei Kindern im Bereich von 22,50 bis 23,50 mm. Die Verteilung wird durch Abbildung 10 verdeutlicht. Die individuellen Werte der Einzelmessungen streuten kaum. Die Standardabweichung der intraindividuellen Einzelwerte 


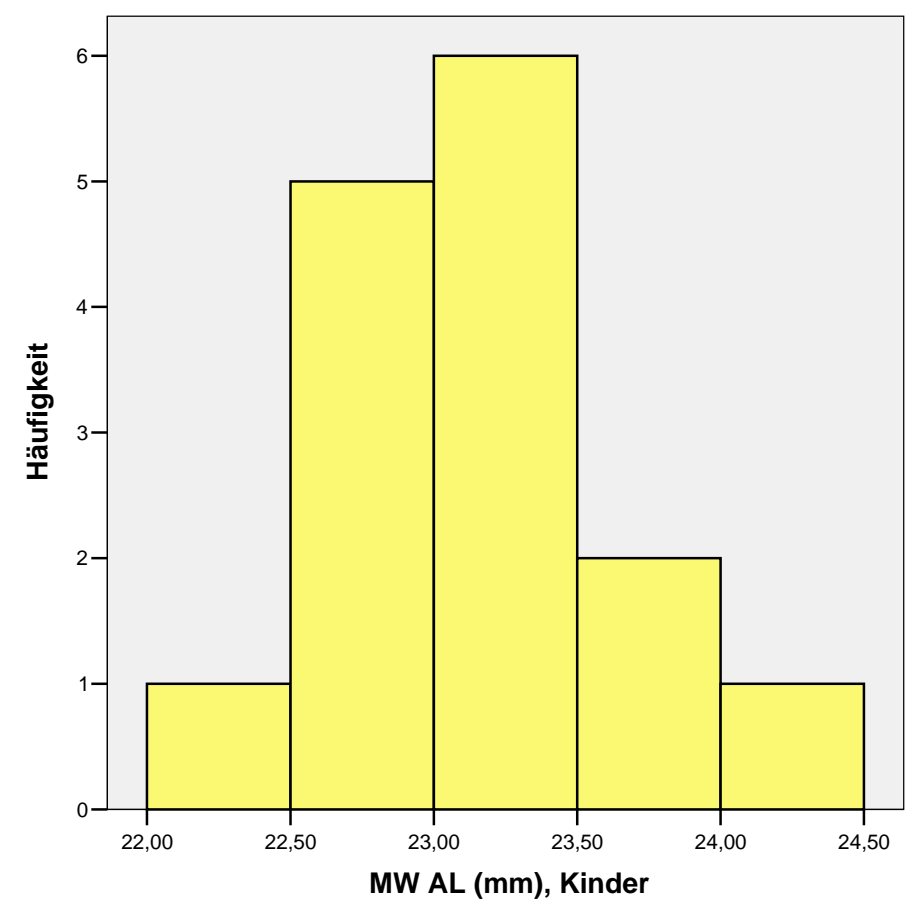

Abbildung 10: Histogramm zur Verteilung der AL bei Kindern

lag in $60 \%(\mathrm{~N}=9)$ bei $0,01 \mathrm{~mm}$, maximal jedoch bei $0,02 \mathrm{~mm}$. Auch die Korrelation zwischen den beiden Augen war hoch $(\mathrm{r}=0,93)$.

\subsubsection{Jugendliche}

In der Gruppe der Jugendlichen wurden, genauso wie bei Erwachsenen und Pseudophaken alle vorgestellten Parameter jeweils viermal gemessen. Es stellte sich im Laufe der Zeit heraus, dass die dieser Gruppe angehörenden Probanden am schnellsten in der Lage waren, sich die Zahlen auf dem Plättchen bzw. Spiegel einzustellen. Die Messungen dauerten bei ihnen daher auch am kürzesten, von 30 Minuten bis zu etwa einer Stunde.

\subsubsection{Vorderkammertiefe}

Die gemessenen Werte der VKT im unakkommodierten Zustand reichten von 3,21 bis 4,21 $\mathrm{mm}$ auf dem rechten, sowie von 3,24 bis 4,21 mm auf dem linken Auge. Es ergab sich eine sehr hohe Korrelation von $\mathrm{r}=0,99$ zwischen den VKT beider Augen. Bei 80\% $(\mathrm{N}=12)$ der Jugendlichen belief sich die VKT im Bereich von 3,60 bis 3,80 mm. 13\% (N=2) der VKT bei den Jugendlichen waren jedoch größer als 4,00 mm. Die Standardabweichung der Einzelwerte auf dem rechten Auge lag bei höchstens 0,02 mm. Dieser Wert wurde bei 27\% (N=4) der jugendlichen Probanden ermittelt. 


\begin{tabular}{|l||l|l|}
\hline & rechtes Auge & linkes Auge \\
\hline MW & 3,76 & 3,77 \\
\hline Median & 3,76 & 3,76 \\
\hline Min & 3,21 & 3,24 \\
\hline Max & 4,21 & 4,21 \\
\hline SD & 0,23 & 0,23 \\
\hline
\end{tabular}

Tabelle 6: Übersicht über VKT bei Jugendlichen, Angaben in mm

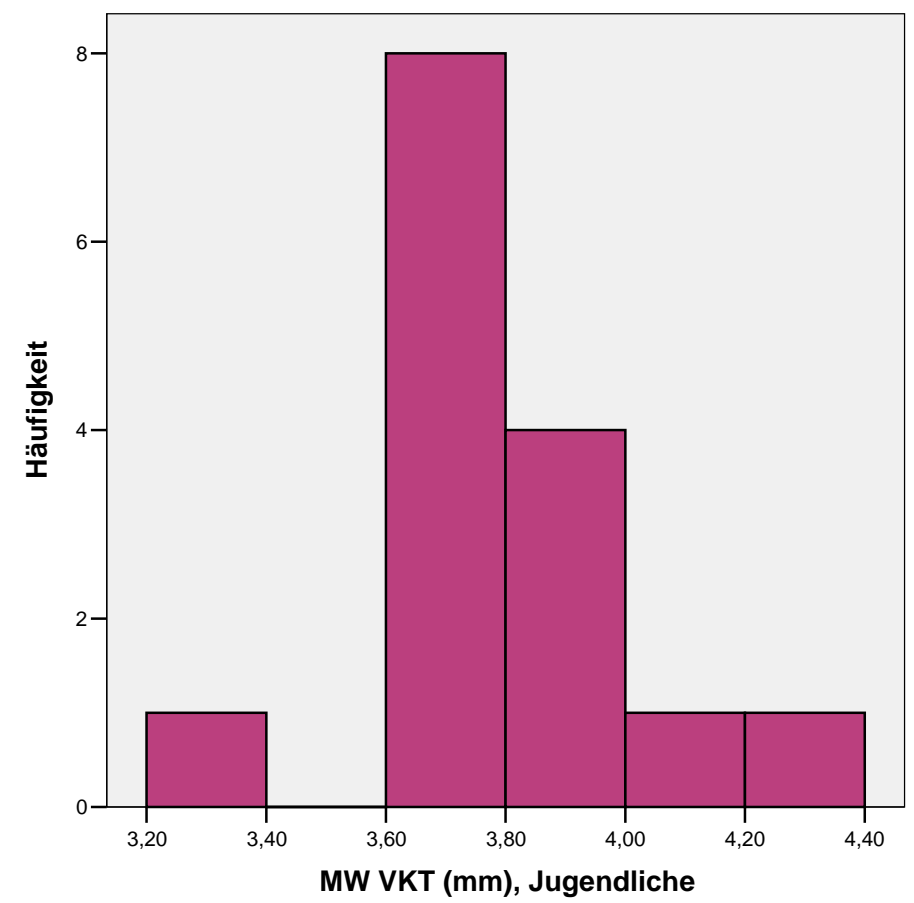

Abbildung 11: Histogramm zur Verteilung der VKT bei Jugendlichen 


\subsubsection{Augenlänge}

Die AL-Werte der Jugendlichen verteilten sich im Bereich von 22,46 bis 25,35 mm auf dem rechten und von 22,39 bis 25,61 mm auf dem linken Auge. Die Korrelation zwischen den beiden Seiten war sehr hoch $(r=0,99)$.

\begin{tabular}{|l||l|l|}
\hline & rechtes Auge & linkes Auge \\
\hline MW & 23,94 & 23,95 \\
\hline Median & 23,76 & 23,91 \\
\hline Min & 22,46 & 22,39 \\
\hline Max & 25,35 & 25,61 \\
\hline SD & 0,79 & 0,86 \\
\hline
\end{tabular}

Tabelle 7: Übersicht über AL bei Jugendlichen, Angaben in mm

Etwa die Hälfte $(\mathrm{N}=8)$ der AL belief sich bei den Jugendlichen jedoch im Bereich von 23,00 bis 24,00 mm, was durch Abbildung 12 verdeutlicht wird. Grundsätzlich ist festzustellen, dass bei Jugendlichen, sowie bei allen anderen Gruppen auch, die Standardabweichung der intraindividuellen Einzelwerte bei den Messungen der AL stets kleiner ausfiel als bei jenen der VKT. Bei Jugendlichen ergab sich in 73\% (N=11) der Fälle eine Streuung der Einzelwerte von $0,01 \mathrm{~mm}$.

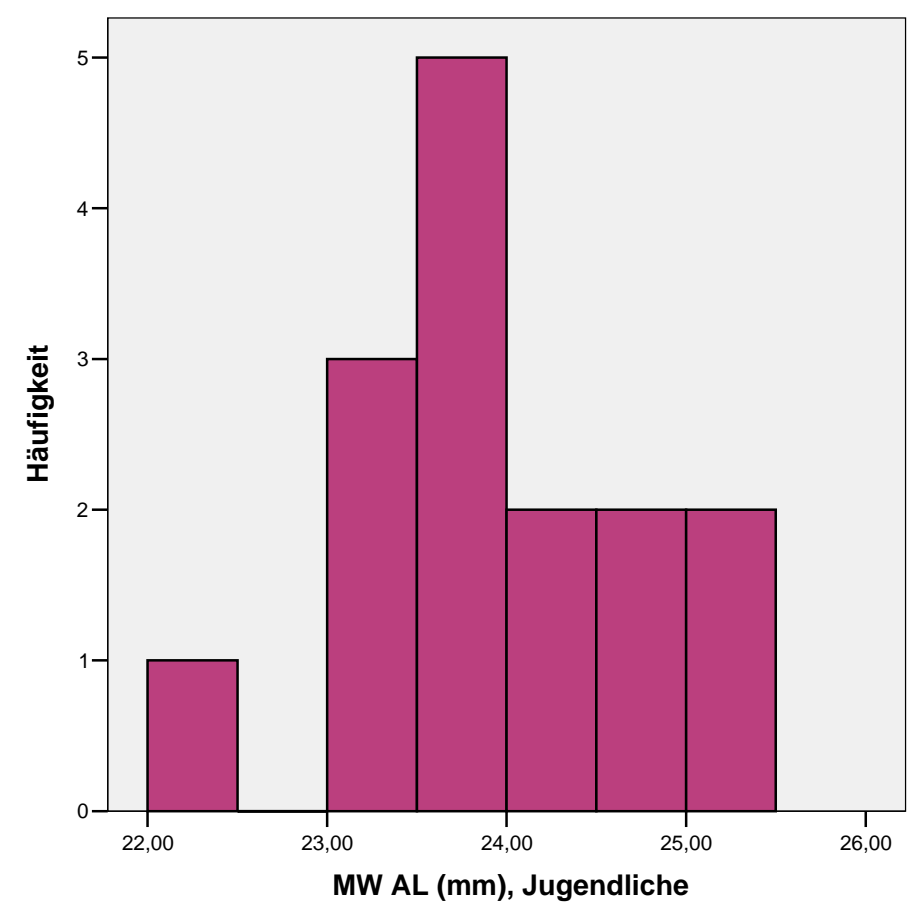

Abbildung 12: Histogramm zur Verteilung der AL bei Jugendlichen 


\subsubsection{Erwachsene}

Die Gruppe der Erwachsenen brauchte im Vergleich zu den Jugendlichen vor allem am Anfang etwas länger, um sich die Zahlen, die auf dem LED-Panelmeter aufleuchteten, auf dem Plättchen, bzw. Spiegel einzustellen. Die Messreihen dauerten bei ihnen daher zwischen 60 und 110 Minuten.

\subsubsection{Vorderkammertiefe}

Die gemittelte VKT rangierte in der Gruppe der Erwachsenen im Bereich von 2,63 bis 3,95 $\mathrm{mm}$ auf dem rechten und von 2,76 bis $3,95 \mathrm{~mm}$ auf dem linken Auge. Die Standardabweichung der intraindividuellen Einzelwerte lag höchstens bei 0,02 mm. Dieser Wert wurde in $27 \%(\mathrm{~N}=4)$ der Fälle errechnet.

Auch bei Erwachsenen ergab sich eine hohe Korrelation zwischen den VKT des rechten und des linken Auges $(r=0,97)$.

\begin{tabular}{|l||l|l|}
\hline & rechtes Auge & linkes Auge \\
\hline MW & 3,21 & 3,25 \\
Median & 3,14 & 3,16 \\
Min & 2,63 & 2,76 \\
Max & 3,95 & 3,95 \\
SD & 0,33 & 0,34 \\
\hline
\end{tabular}

Tabelle 8: Übersicht über VKT bei Erwachsenen, Angaben in mm

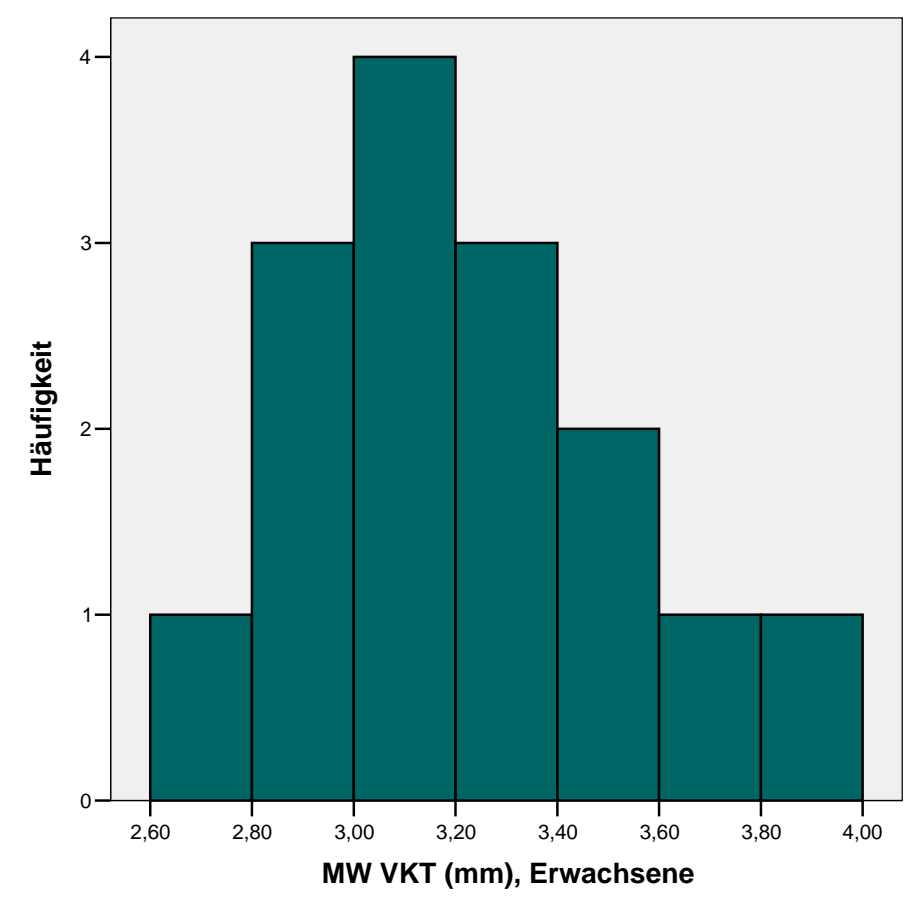

Abbildung 13: Histogramm zur Verteilung der VKT bei Erwachsenen 


\subsubsection{Augenlänge}

Die Mittelwerte der AL im unakkommodierten Zustand beliefen sich in der Gruppe der Erwachsenen im Bereich von 22,63 bis 25,33 mm auf dem rechten und von 22,61 bis 25,14 mm auf dem linken Auge.

\begin{tabular}{|l||l|l|}
\hline & rechtes Auge & linkes Auge \\
\hline MW & 23,66 & 23,64 \\
Median & 23,70 & 23,61 \\
Min & 22,63 & 22,61 \\
Max & 25,33 & 25,14 \\
SD & 0,73 & 0,69 \\
\hline
\end{tabular}

Tabelle 9: Übersicht über AL bei Erwachsenen, Angaben in mm

Die Einzelwerte der jeweiligen Probanden streuten kaum. In 67\% ( $N=10)$ der Fälle wurde eine Standardabweichung von lediglich $0,01 \mathrm{~mm}$ ermittelt.

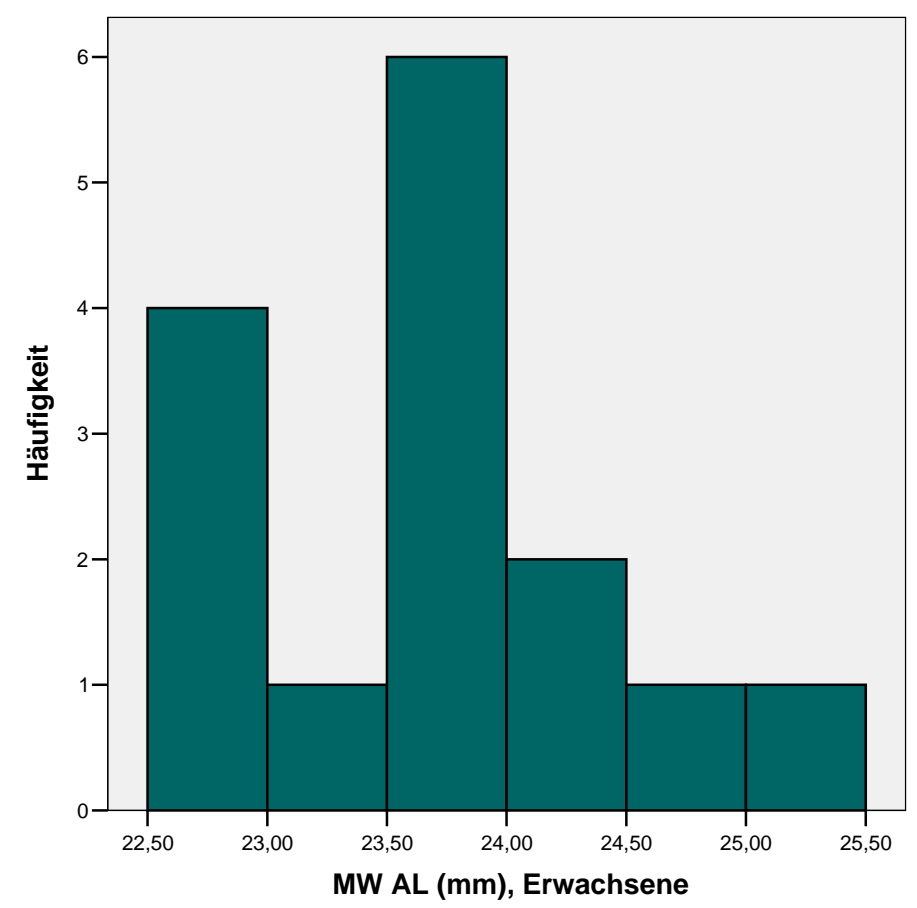

Abbildung 14: Histogramm zur Verteilung der AL bei Erwachsenen 


\subsubsection{Pseudophake Erwachsene}

Den Ergebnissen der Messungen im unakkommodierten Zustand muss in der Gruppe der pseudophaken Erwachsenen vorangestellt werden, dass die Bedienungsanleitung des IOLMasters vorschlägt, den Modus der VKT in diesem Kollektiv nicht anzuwenden. Dennoch ist dieses im klinischen Alltag üblich und wurde auch in dieser Studie durchgeführt.

\subsubsection{Vorderkammertiefe}

Die Werte der VKT rangierten bei den Pseudophaken im Bereich von 3,46 bis 4,17 mm.

\begin{tabular}{|l||l|}
\hline & $\begin{array}{l}\text { Pseudophakes } \\
\text { Auge }\end{array}$ \\
\hline MW & 3,90 \\
\hline Median & 3,93 \\
\hline Min & 3,46 \\
\hline Max & 4,17 \\
\hline SD & 0,20 \\
\hline
\end{tabular}

Tabelle 10: Übersicht über VKT bei Pseudophaken, Angaben in mm

Dabei wurden bei dieser Gruppe im Vergleich deutlich höhere Standardabweichungen der intraindividuellen Einzelwerte errechnet. Diese lagen in 13\% $(\mathrm{N}=2)$ der Fälle sogar bei 0,05 $\mathrm{mm}$.

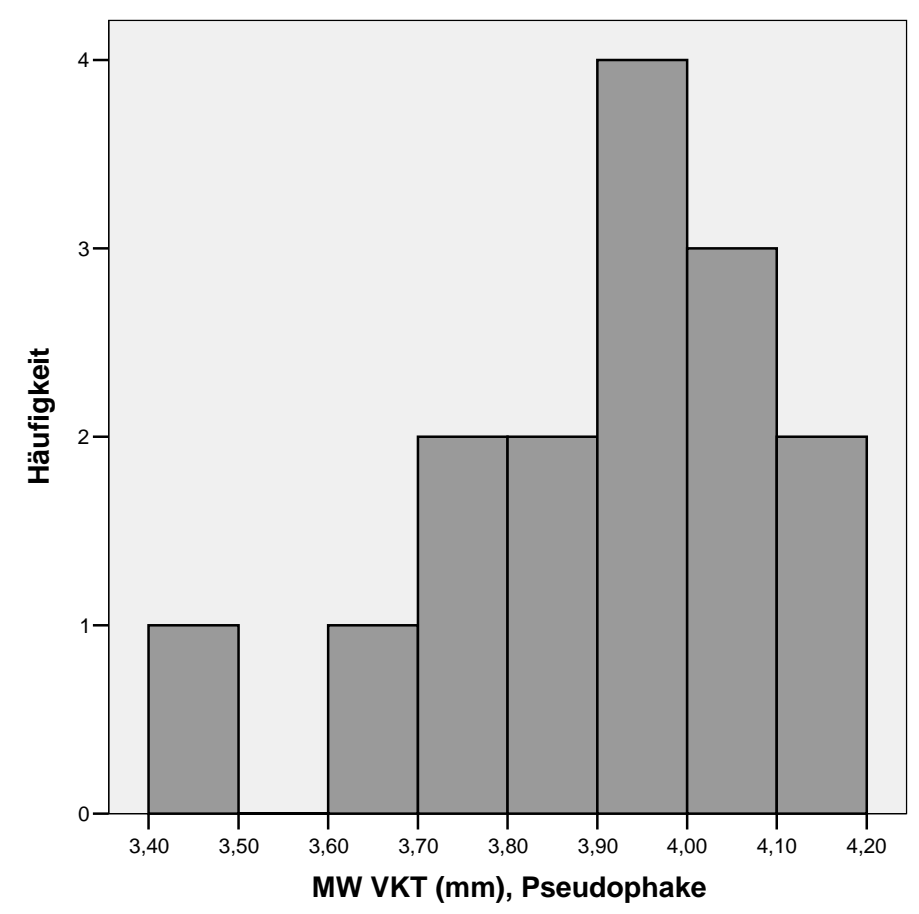

Abbildung 15: Histogramm zur Verteilung der VKT bei Pseudophaken 


\subsubsection{Augenlänge}

Die AL verteilte sich in der Gruppe der Pseudophaken im Bereich von 21,44 bis 24,25 mm, wobei etwa die Hälfte (N=7) der Probanden AL zwischen 23,00 und 24,00 mm aufwies.

Auch hier zeigten sich die Einzelmessungen der AL sehr konstant. In 80\% (N=12) der Fälle lag die intraindividuelle Standardabweichung lediglich bei $0,01 \mathrm{~mm}$.

\begin{tabular}{|l||l|}
\hline & $\begin{array}{l}\text { pseudophakes } \\
\text { Auge }\end{array}$ \\
\hline MW & 23,05 \\
Median & 23,11 \\
\hline Min & 21,44 \\
Max & 24,25 \\
SD & 0,79 \\
\hline
\end{tabular}

Tabelle 11: Übersicht über AL bei Pseudophaken, Angaben in mm

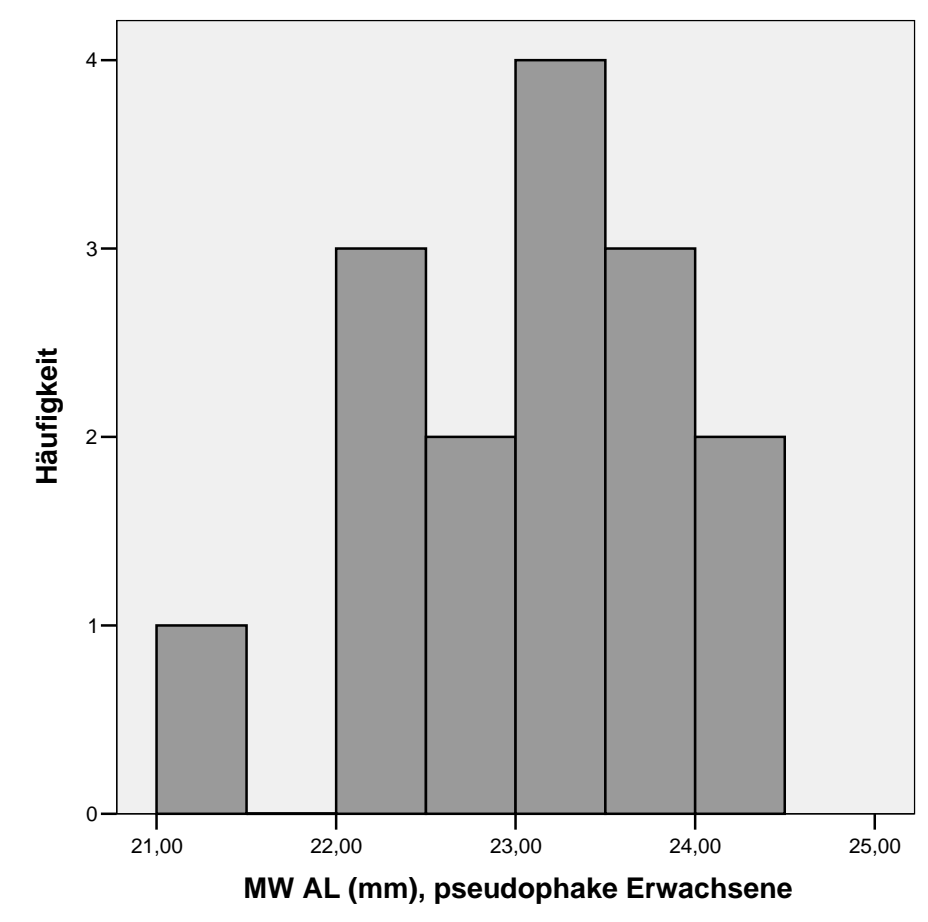

Abbildung 16: Histogramm zur Verteilung der AL bei Pseudophaken

\subsection{Veränderungen während der Akkommodation}

Im Folgenden wird nun darauf eingegangen wie sich die gemessenen Parameter, also VKT und AL, bei Präsentation der Akkommodationsstimuli in den einzelnen Gruppen verändern. 
Dabei werden aus Gründen der Übersicht in diesem Kapitel nur die Messungen am rechten Auge mit der Plättchen-Methode in Tabellen und Grafiken berücksichtigt.

\subsubsection{Veränderungen der Vorderkammertiefe}

Zunächst einmal werden die Änderungen der VKT im Rahmen der Akkommodationsantwort besprochen. Bei allen Gruppen ergab sich eine deutliche Verkürzung der VKT bei Nahakkommodation im Vergleich zur Messung ohne Akkommodationsreiz. Gemeinsam war ihnen ebenfalls die Tatsache, dass die Werte der Fernakkommodation und die der Messungen ohne jeglichen Akkommodationsstimulus nahe beieinander lagen.

\subsubsection{Kinder}

In Tabelle 12 wird die deskriptive Statistik für gemittelte VKT bei Nah- und Fernakkommodation vorgestellt. Die VKT verkürzte sich bei Kindern signifikant $(p=0,00)$, sowohl bei Messungen mit Plättchen als auch mit Spiegel bei Nahakkommodation im Vergleich zum unstimulierten Zustand.

\begin{tabular}{|l||l|l|}
\hline & Nahakkommodation & Fernakkommodation \\
\hline MW & 3,51 & 3,63 \\
\hline Median & 3,49 & 3,62 \\
\hline Min & 3,22 & 3,34 \\
\hline Max & 3,91 & 3,98 \\
\hline SD & 0,20 & 0,19 \\
\hline
\end{tabular}

Tabelle 12: VKT (mm) bei Nah- und Fernakkommodation, Kinder

In Abbildung 17 wird die Häufigkeit der bei Kindern gemessenen Differenzen der VKT zwischen unstimuliertem und nahakkommodiertem Auge verdeutlicht.

Die Veränderung der VKT während der Akkommodation wird in Abbildung 18 dargestellt. Bei Nahakkommodation ergaben sich im Durchschnitt Verkürzungen der VKT von 0,11 mm. Das Maximum lag bei der Spiegel-Methode sogar bei 0,18 mm. Bei der Fernakkommodation konnte man in 33\% (N=5) der Fälle eine Vertiefung der VKT um 0,01 mm messen, bei der Mehrheit konnte jedoch gar keine Veränderung im Vergleich zum unakkommodierten Zustand gemessen werden. 


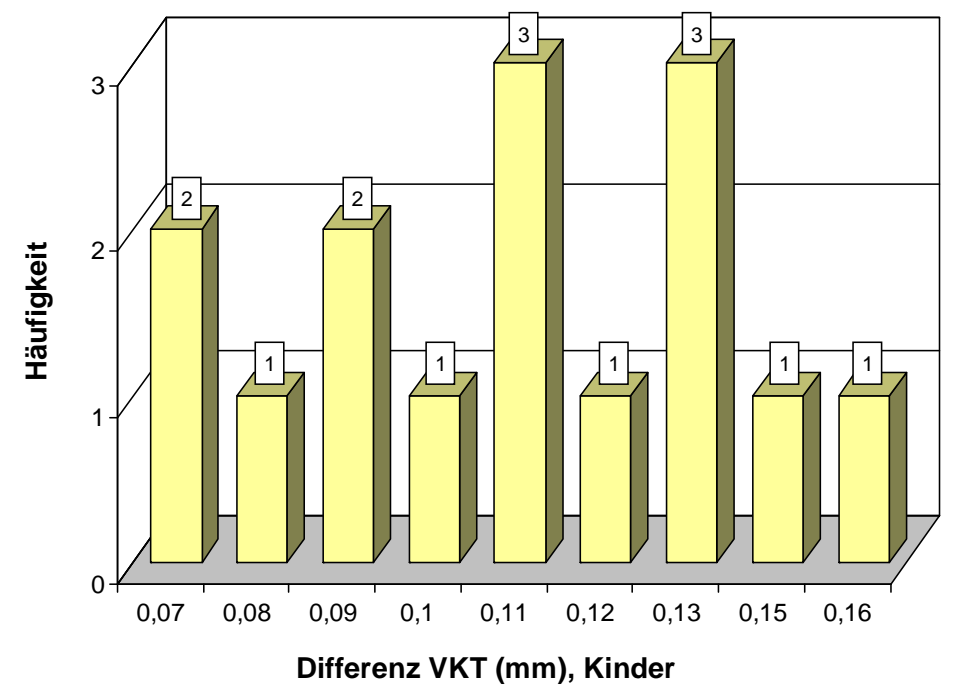

Abbildung 17: Säulendiagramm zur Differenz VKT

(unakkommodiertes - nahakkommodiertes rechtes Auge), Kinder

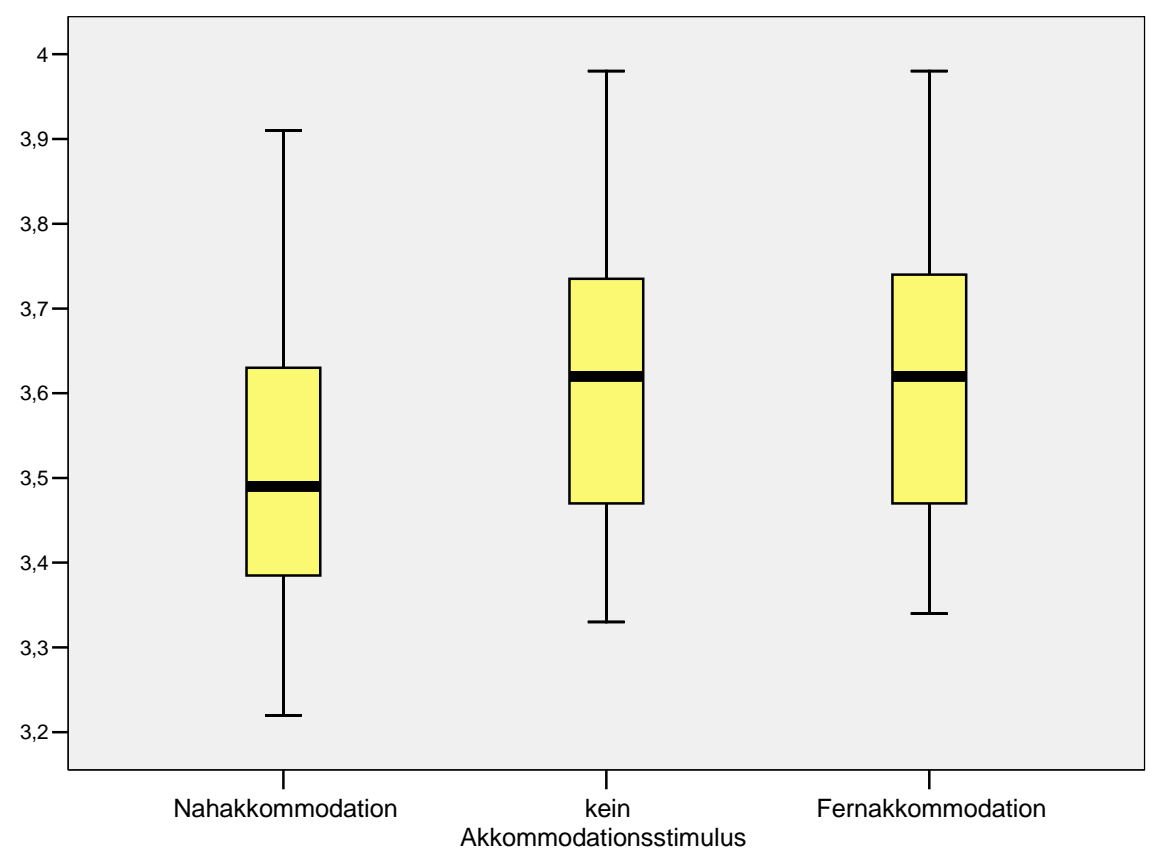

Abbildung 18: Boxplot zur VKT (mm) bei Akkommodation, Kinder 


\subsubsection{Jugendliche}

In der Gruppe der Jugendlichen konnte ebenfalls eine signifikante Verkürzung der VKT bei Nahakkommodation, sowohl bei den Messungen mit Plättchen, als auch bei jenen mit Spiegel $(p=0,00)$ ermittelt werden. Bei der Fernakkommodation hingegen konnte kein signifikanter Unterschied zum unakkommodierten Zustand gezeigt werden, weder mit Plättchen $(p=1,00)$, noch mit Spiegel $(p=0,66)$.

\begin{tabular}{|l||l|l|}
\hline & Nahakkommodation & Fernakkommodation \\
\hline MW & 3,62 & 3,76 \\
\hline Median & 3,59 & 3,76 \\
\hline Min & 3,05 & 3,21 \\
\hline Max & 4,09 & 4,21 \\
\hline SD & 0,23 & 0,23 \\
\hline
\end{tabular}

Tabelle 13: VKT (mm) bei Nah- und Fernakkommodation, Jugendliche

Während der Nahakkommodation ergaben sich bei Jugendlichen im Schnitt Verkürzungen der VKT um 0,14 mm. Bei den Messungen mit Plättchen lag das Maximum der Differenz sogar bei 0,20 mm, wie auch aus Abbildung 19 deutlich wird.

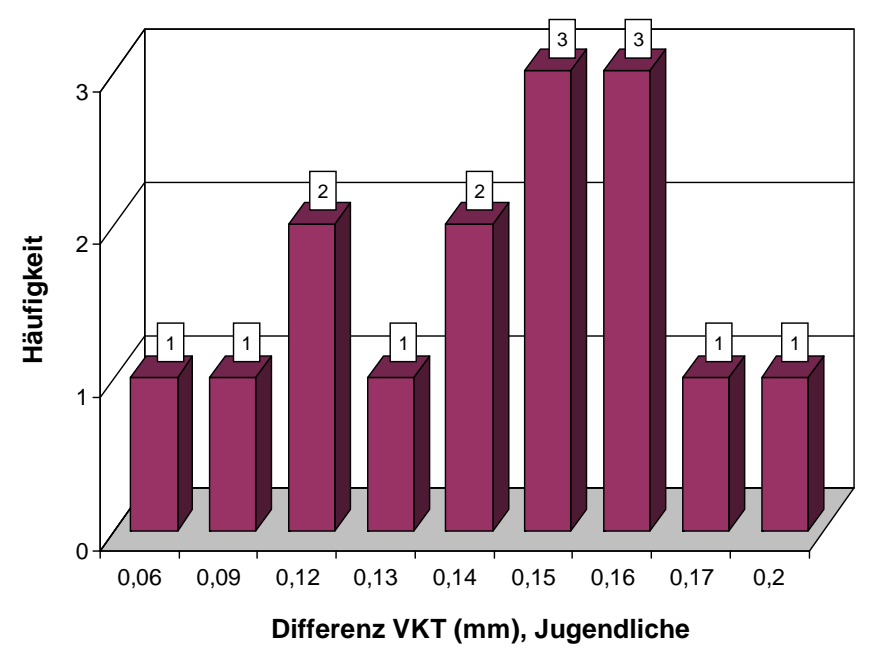

Abbildung 19: Säulendiagramm zur Differenz VKT

(unakkommodiertes - nahakkommodiertes rechtes Auge), Jugendliche

Während der Fernakkommodation wurden in der Gruppe der Jugendlichen sowohl eine Vertiefung der VKT bis hin zu 0,05 mm im Vergleich zum unakkommodierten Zustand, aber in $13 \%(\mathrm{~N}=2)$ der Fälle auch eine leichte Verflachung der VKT gemessen. Bei knapp der 
Hälfte der Probanden (N=9) konnte jedoch gar keine Änderung der VKT im Vergleich zur Messung ohne Akkommodationsstimulus ausgemacht werden. Die Verteilung der VKT bei Jugendlichen, mit und ohne Akkommodation, wird in Abbildung 20 dargestellt.

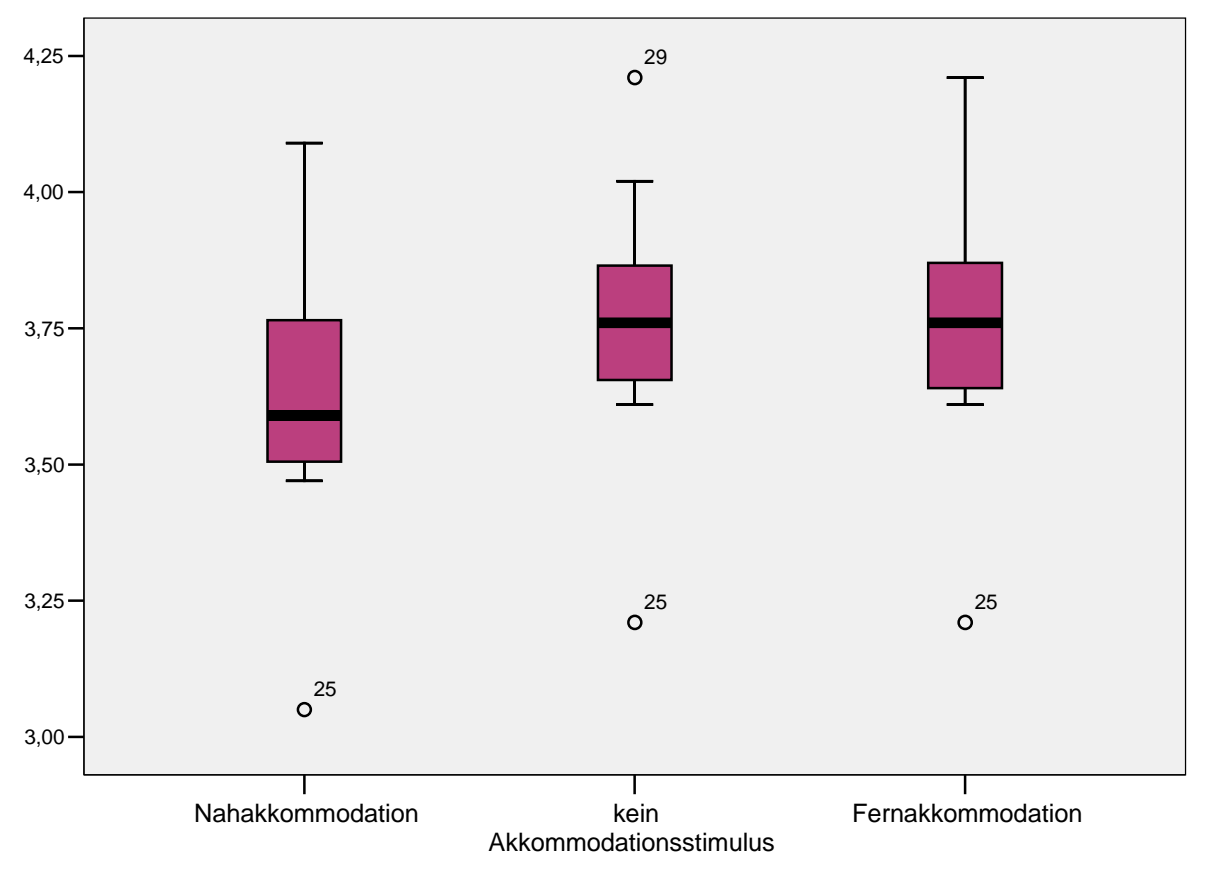

Abbildung 20: Boxplot zur VKT (mm) bei Akkommodation, Jugendliche

\subsubsection{Erwachsene}

Vergleichbar zu den ersten beiden Gruppen, ergaben sich auch bei Erwachsenen signifikante Veränderungen der VKT bei Nahakkommodation im Vergleich zum unakkommodierten Zustand $(\mathrm{p}=0,00)$.

\begin{tabular}{|l||l|l|}
\hline & Nahakkommodation & Fernakkommodation \\
\hline MW & 3,15 & 3,22 \\
\hline Median & 3,10 & 3,15 \\
Min & 2,53 & 2,64 \\
Max & 3,90 & 3,95 \\
SD & 0,34 & 0,33 \\
\hline
\end{tabular}

Tabelle 14: VKT (mm) bei Nah- und Fernakkommodation, Erwachsene

Die Verkürzung der VKT bei Nahakkommodation betrug bei Erwachsenen im Durchschnitt 0,07 mm. Wie die in Abbildung 21 dargestellte Verteilung schon erkennen lässt, betrug die Änderung der VKT in 20\% (N=3) der Fälle lediglich 0,04 mm, das Maximum lag bei 0,11 $\mathrm{mm}$. 


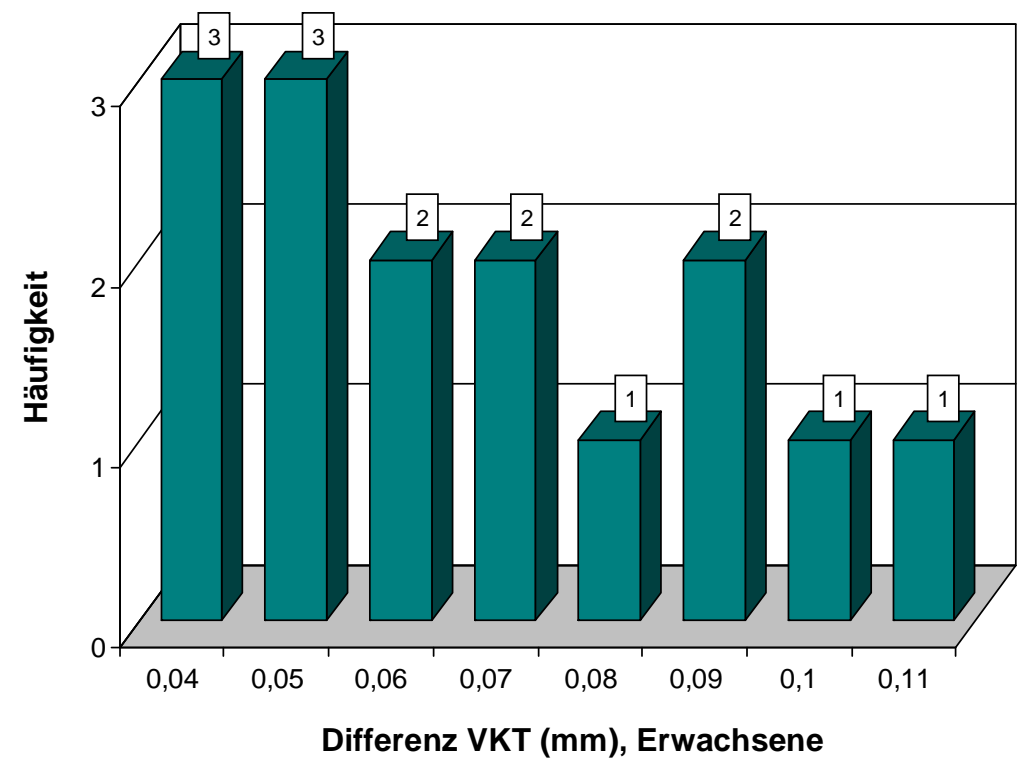

Abbildung 21: Säulendiagramm zur Diferenz VKT (unakkommodiertes - nahakkommodiertes rechtes Auge), Erwachsene

Bei der Fernakkommodation ergab sich bei beiden Methoden in etwa der Hälfte der Fälle eine leichte Vertiefung der VKT von $0,01 \mathrm{~mm}$. Diese war jedoch weder mit der Plättchen- $(\mathrm{p}=0,06)$ noch mit der Spiegel- Methode $(\mathrm{p}=0,58)$ signifikant.

Die Verteilung der VKT bei Nah- und Fernakkommodation im Vergleich zum unakkommodierten Zustand wird für die Gruppe der Erwachsenen in Abbildung 22 verdeutlicht.

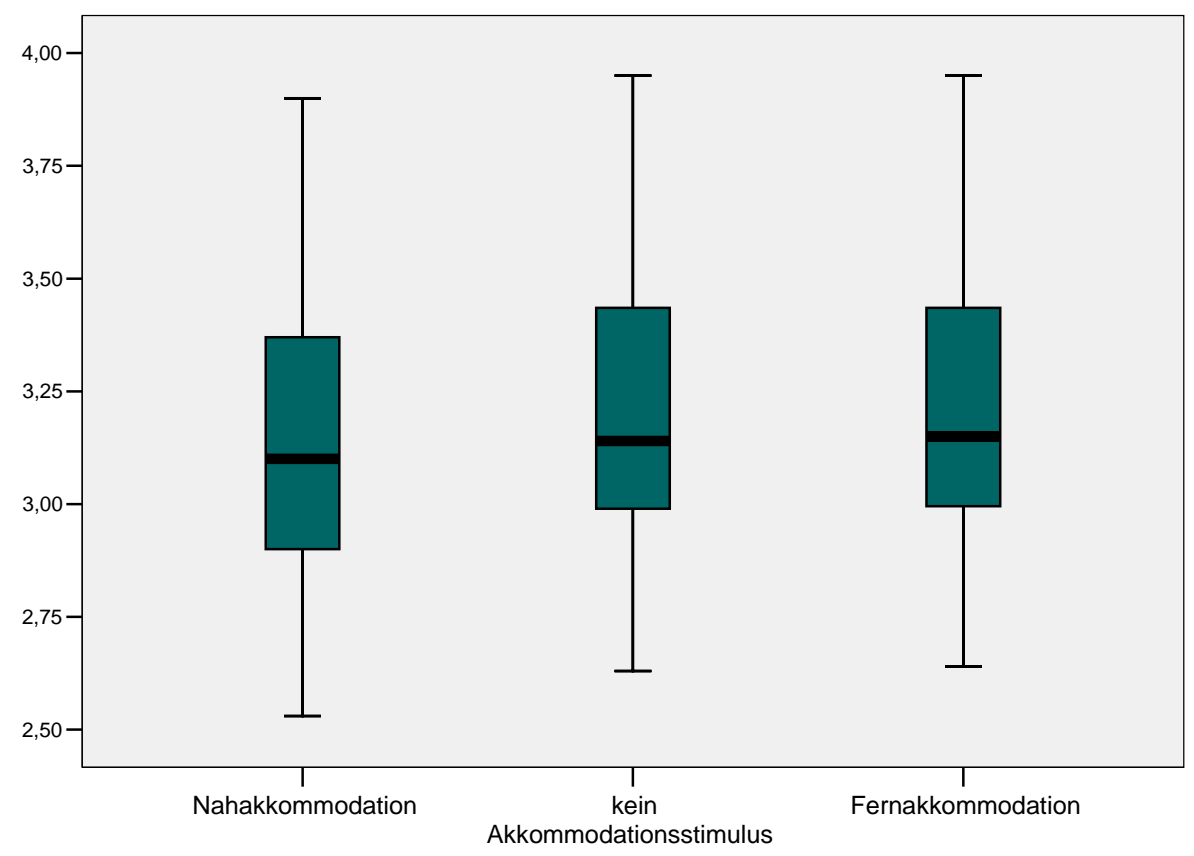

Abbildung 22: Boxplot zur VKT (mm) bei Akkommodation, Erwachsene 


\subsubsection{Pseudophake Erwachsene}

Die pseudophaken Erwachsenen unterscheiden sich bei Betrachtung der Änderung der VKT während der Akkommodation deutlich von allen anderen Gruppen.

\begin{tabular}{|l||l|l|}
\hline & Nahakkommodation & Fernakkommodation \\
\hline MW & 3,90 & 3,90 \\
\hline Median & 3,92 & 3,93 \\
\hline Min & 3,46 & 3,47 \\
\hline Max & 4,16 & 4,17 \\
\hline SD & 0,20 & 0,19 \\
\hline
\end{tabular}

Tabelle 15: VKT (mm) bei Nah- und Fernakkommodation, Pseudophake

Bei der Nahakkommodation mit Plättchen wurde, wie Abbildung 23 deutlich macht, in $80 \%$ $(\mathrm{N}=12)$ der Fälle gar keine Änderung im Vergleich zum unakkommodierten Zustand gemessen. Bei der Spiegel-Methode waren es ebenfalls noch $60 \%(\mathrm{~N}=9)$ der Fälle ohne jegliche Veränderung der VKT, bei $40 \%(\mathrm{~N}=6)$ der Probanden wurde hierbei aber eine leichte Verkürzung der VKT um $0,01 \mathrm{~mm}$ festgestellt.

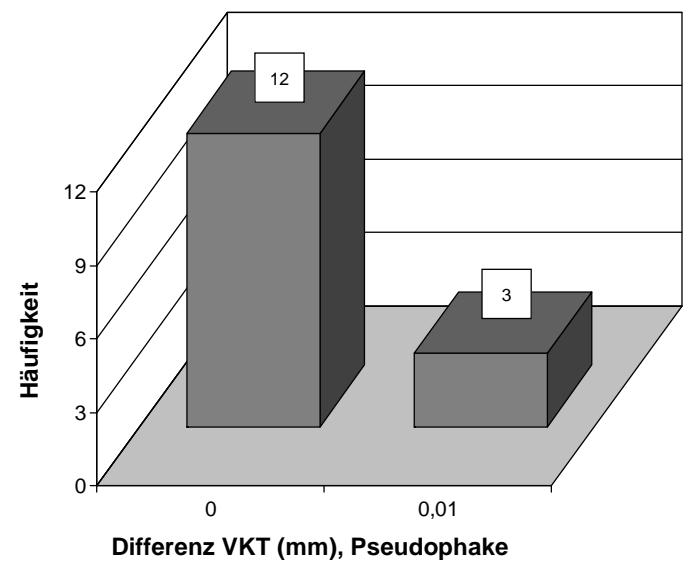

Abbildung 23: Säulendiagramm zur Differenz VKT

(unakkommodiertes - nahakkommodiertes rechtes Auge), Pseudophake

Insgesamt konnte also mit beiden Methoden bei der Mehrzahl der Fälle gar keine Bewegung der Linse bei Nahakkommodation an einer Änderung der VKT nachvollzogen werden. Ebenso wenig ließ sich statistisch im zweiseitigen t-Test für verbundene Stichproben eine 


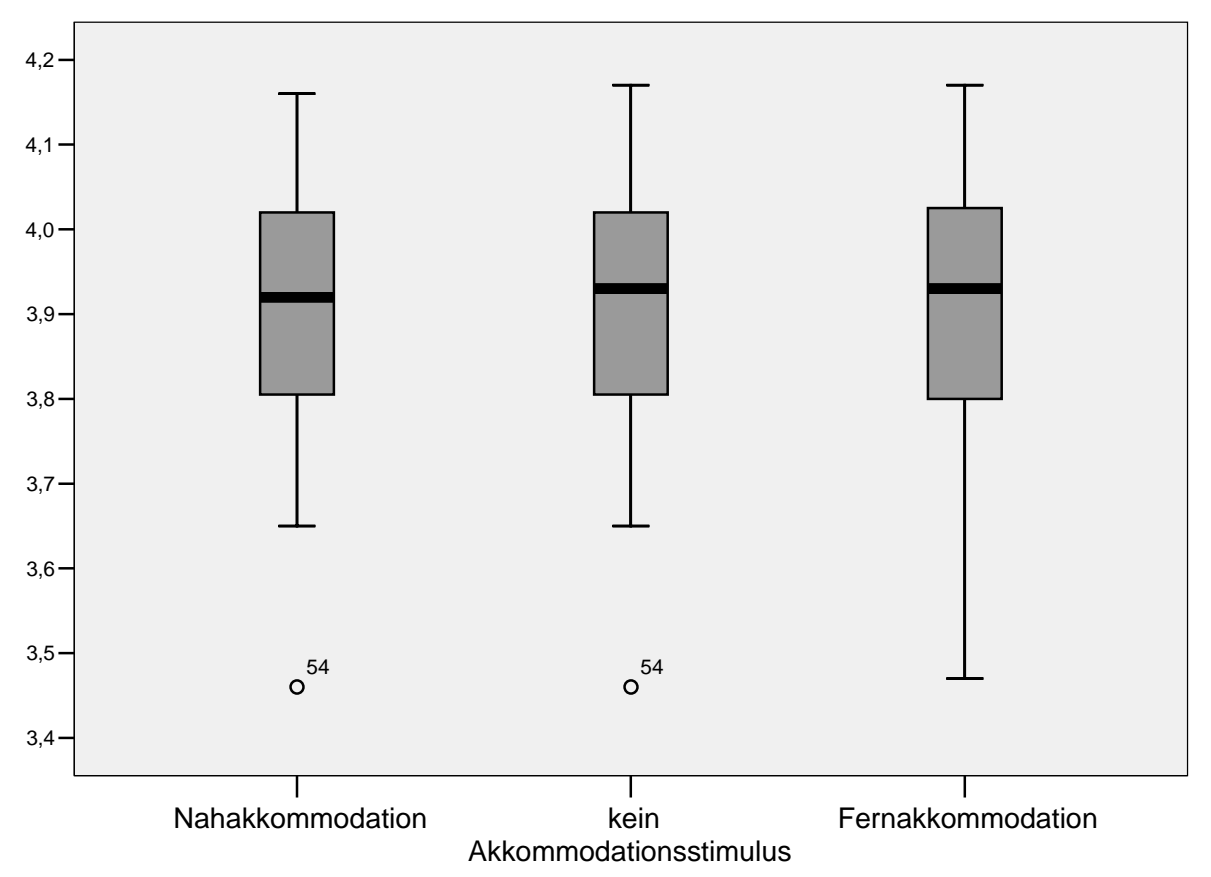

Abbildung 24: Boxplot zur VKT (mm) bei Akkommodation, Pseudophake

signifikante Änderung der VKT bei der Plättchen-Methode errechnen $(p=0,08)$. In der Spiegel- Versuchsreihe war die Verkürzung der VKT um $0,01 \mathrm{~mm}$, die bei $40 \%$ der Probanden gemessen wurde, statistisch signifikant $(p=0,01)$.

Was die Messungen bei Fernakkommodation betrifft, so unterscheidet sich diese Gruppe nicht wesentlich von den anderen. Bei 73\% (N=11) der Probanden konnte keine Änderung der VKT bei Fokussierung auf die in der Ferne gelegenen Zahlen im Vergleich zum unstimulierten Zustand bei den Messungen mit Plättchen ermittelt werden. In 36\% (N=4) der Fälle ergab sich sogar eine leichte Verkürzung von 0,01 mm. Weder mit der Plättchen$(p=0,33)$ noch mit der Spiegel-Methode $(p=0,16)$ war der Unterschied hierbei jedoch signifikant. Die Verteilung der VKT bei Akkommodation wird für die Gruppe der Pseudophaken durch Abbildung 24 verdeutlicht.

\subsubsection{Veränderungen der Augenlänge}

Besonderes Augenmerk wurde in dieser Arbeit auf die Messung der AL gelegt. Ihre Änderung mit der Akkommodation gestaltete sich in allen vier Gruppen ähnlich. Dies ist bereits anhand des Fehlerbalkens in Abbildung 25, an dem man Mittelwerte und Extrema der drei verschiedenen Akkommodationszustände ablesen kann, gut zu erkennen, und soll im Folgenden für die verschiedenen Gruppen erörtert werden. 


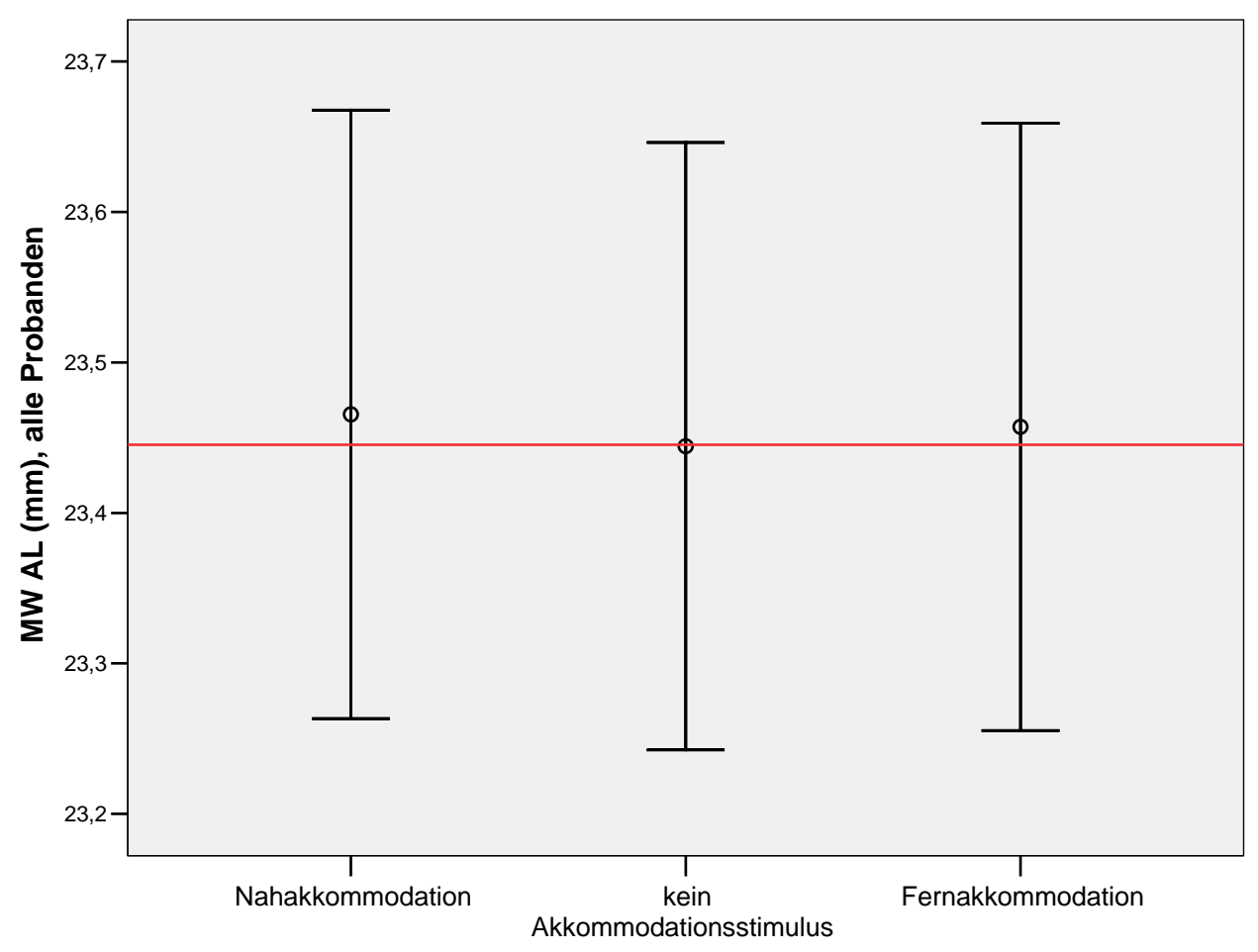

Abbildung 25: Fehlerbalken zur AL bei Akkommodation, alle Probanden

\subsubsection{Kinder}

Bei Kindern konnte in 46\% (N=7) der Fälle eine Zunahme der Augenlänge von $0,01 \mathrm{~mm}$ bei Nahakkommodation mit Plättchen gemessen werden.

Bei einem genauso großen Anteil konnte allerdings gar keine Veränderung festgestellt werden. Dieser Zusammenhang wird in Abbildung 26 dargelegt.

Bei den Messungen mit dem Spiegel ergab sich jedoch bei $73 \%(\mathrm{~N}=11)$ der Kinder eine signifikante Zunahme der AL bei Nahakkommodation $(\mathrm{p}=0,00)$.

\begin{tabular}{|l||l|l|}
\hline & Nahakkommodation & Fernakkommodation \\
\hline MW & 23,19 & 23,19 \\
\hline Median & 23,29 & 23,29 \\
\hline Min & 22,46 & 22,45 \\
\hline Max & 24,35 & 24,35 \\
\hline SD & 0,49 & 0,49 \\
\hline
\end{tabular}

Tabelle 16: AL (mm)bei Nah- und Fernakkommodation, Kinder 


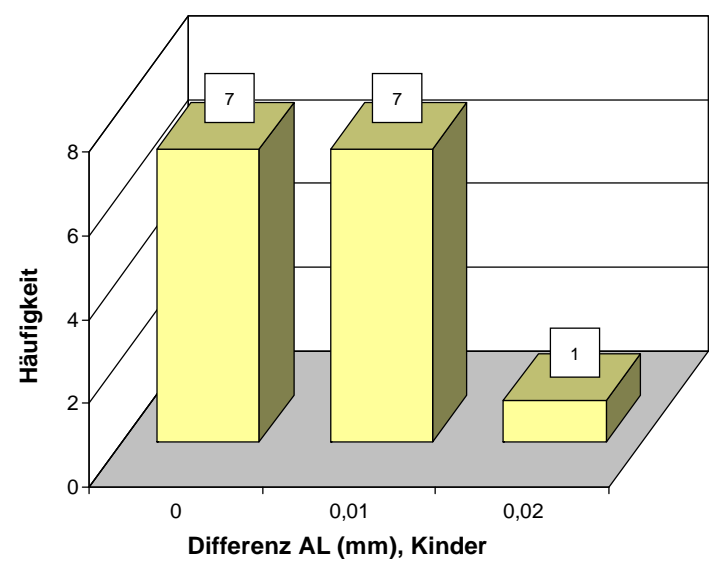

Abbildung 26: Säulendiagramm zur Differenz AL

(nahakkommodiertes - unakkommodiertes rechtes Auge), Kinder

Was die Fernakkommodation betrifft, so konnte weder bei der Versuchsreihe mit Spiegel noch bei der mit Plättchen eine signifikante Änderung nachgewiesen werden $(p>0,63)$. Bei Fernakkommodation mit Plättchen änderte sich in 100\% $(\mathrm{N}=15)$, bei jener mit Spiegel in 93\% $(\mathrm{N}=14)$ der Fälle die AL überhaupt nicht im Vergleich zum unstimulierten Zustand.

\subsubsection{Jugendliche}

In der Gruppe der Jugendlichen konnte sowohl mit Plättchen als auch mit Spiegel eine signifikante Verlängerung der AL bei Nahakkommodation beobachtet werden $(p=0,00)$.

\begin{tabular}{|l||l|l|}
\hline & Nahakkommodation & Fernakkommodation \\
\hline MW & 23,95 & 23,94 \\
\hline Median & 23,76 & 23,75 \\
\hline Min & 22,47 & 22,46 \\
\hline Max & 25,37 & 25,35 \\
\hline SD & 0,79 & 0,79 \\
\hline
\end{tabular}

Tabelle 17: AL (mm) bei Nah- und Fernakkommodation, Jugendliche

Bei $67 \%(\mathrm{~N}=10)$ der Probanden verlängerte sich die AL bei Nahakkommodation und Verwendung der Plättchen-Methode. Bei Betrachtung der nahen Zahlen mit Spiegel konnte sogar in $87 \%(\mathrm{~N}=13)$ der Fälle eine leichte Änderung der AL gemessen werden, meistens $(\mathrm{N}=9)$ in der Größenordnung von 0,01 . 


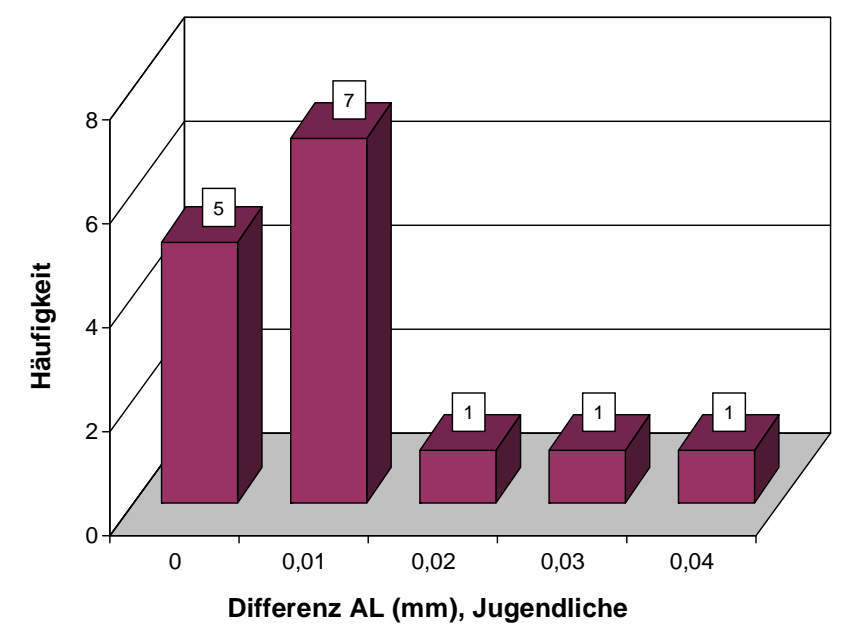

Abbildung 27: Säulendiagramm zur Differenz AL (nahakkommodiertes - unakkommodiertes rechtes Auge), Jugendliche

Die Akkommodationsantwort beim Blick in die Ferne ließ sich auch in dieser Gruppe nicht an einer Veränderung der AL nachvollziehen ( $p=0,27$ für Plättchen und $p=0,69$ für Spiegel). In 73\% (N=11) der Fälle bei der Versuchsreihe mit Plättchen und bei 87\% (N=13) der Probanden bei jener mit Spiegel konnte keinerlei Änderung bei Fernakkommodation im Vergleich zum unakkommodierten Zustand gemessen werden.

\subsubsection{Erwachsene}

In der Gruppe der Erwachsenen wurde bei 6 der 15 Probanden (40\%) gar keine Änderung der AL bei Nahakkommodation gemessen, wie in Abbildung 28 deutlich wird.

Bei den anderen neun Probanden wurde allerdings eine kleine (0,01 bis 0,02 mm), wenn auch signifikante ( $p=0,01$ für Plättchen und $p=0,00$ für Spiegel) Verlängerung der AL beim Blick auf die nahen Zahlen beobachtet.

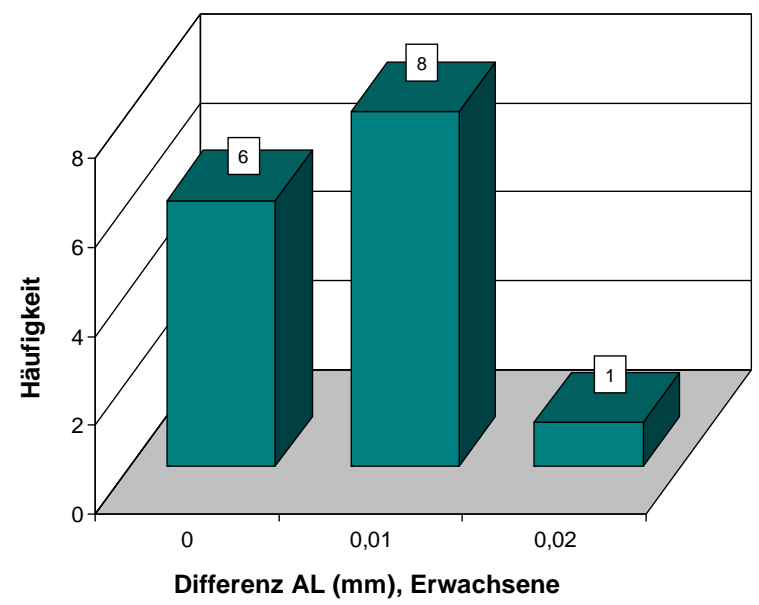

Abbildung 28: Säulendiagramm zur Differenz AL (nahakkommodiertes - unakkommodiertes rechtes Auge), Erwachsene 
Bei Fernakkommodation konnte jedoch auch bei Erwachsenen keine signifikante Änderung der AL, weder in der Messreihe mit Plättchen $(p=0,16)$, noch in jener mit Spiegel $(p=0,53)$ ermittelt werden. Im Einzelnen waren es 87\% (N=13) der Probanden bei der Anordnung mit Plättchen und sogar 93\% (N=14) bei der Messungen mit Spiegel, die keine Änderung der AL bei Fernakkommodation im Vergleich zum unakkommodierten Auge aufwiesen.

Die deskriptive Statistik zur AL bei Nah- und Fernakkomodation bei Erwachsenen wird in Tabelle 18 dargelegt.

\begin{tabular}{|l||l|l|}
\hline & Nahakkommodation & Fernakkommodation \\
\hline MW & 23,67 & 23,66 \\
\hline Median & 23,71 & 23,70 \\
\hline Min & 22,63 & 22,63 \\
\hline Max & 25,34 & 25,33 \\
\hline SD & 0,73 & 0,73 \\
\hline
\end{tabular}

Tabelle 18: AL (mm) bei Nah- und Fernakkommodation, Erwachsene

\subsubsection{Pseudophake Erwachsene}

Der t-Test für gepaarte Stichproben ergab in der Gruppe der Pseudophaken, analog zu den bisher vorgestellten Gruppen, sowohl bei der Plättchen- $(p=0,00)$ als auch bei der SpiegelMethode $(p=0,01)$ eine signifikante Änderung der AL bei Nahakkommodation.

\begin{tabular}{|l||l|l|}
\hline & Nahakkommodation & Fernakkommodation \\
\hline MW & 23,05 & 23,04 \\
\hline Median & 23,12 & 23,11 \\
\hline Min & 21,45 & 21,44 \\
\hline Max & 24,26 & 24,25 \\
\hline SD & 0,79 & 0,79 \\
\hline
\end{tabular}

Tabelle 19: AL (mm) bei Nah- und Fernakkommodation, Pseudophake

In 8, bzw. 9 der 15 Fälle (53\% bzw. 60\%) bei Plättchen und Spiegel konnte eine Verlängerung der AL bei Fokussierung auf die nahen Ziffern gemessen werden.

Nichtsdestotrotz konnte auch hier keine signifikante Änderung der AL beim Blick in die Ferne im Vergleich zur Messung ohne Akkommodationsstimulus nachgewiesen werden. Bei 73\% ( $\mathrm{N}=11)$, bzw. 80\% ( $=12)$ der pseudophaken Probanden konnte weder mit Plättchen noch mit Spiegel eine Änderung der AL bei Fernakkommodation gemessen werden. 


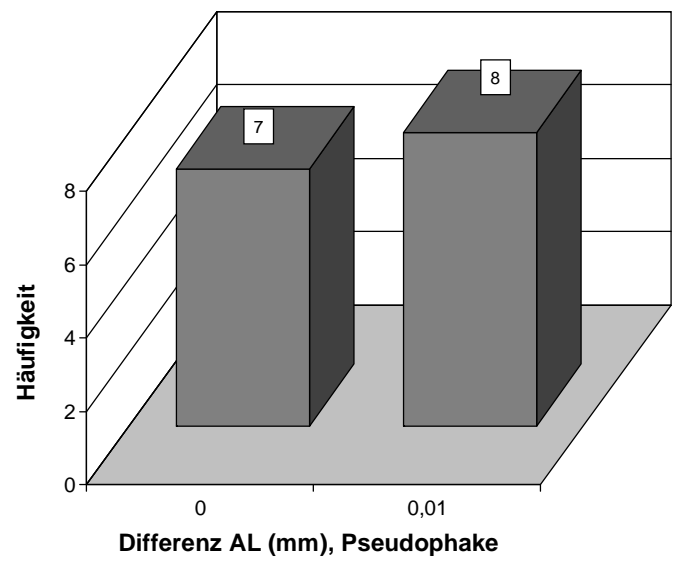

Abbildung 29: Säulendiagramm zur Diferenz AL

(nahakkommodiertes - unakkommodiertes rechtes Auge), Pseudophake

\subsection{Intergruppenvergleiche}

Im kommenden Kapitel sollen nun die gemessenen Merkmale im Hinblick auf Gemeinsamkeiten und Unterschiede zwischen den einzelnen Gruppen analysiert werden. Zur Verdeutlichung der Zusammenhänge werden die Mediane, Quantile und Extreme der Messungen der einzelnen Gruppen in Form von Boxplots vergleichend nebeneinander aufgetragen.

\subsubsection{Vorderkammertiefe}

Wenn man die Verteilung der VKT im Intergruppenvergleich betrachtet, so fällt auf, dass die deutlich kürzesten VKT bei erwachsenen Probanden gemessen wurden.

\begin{tabular}{|l||l|l|l|l|}
\hline & Kinder & Jugendliche & Erwachsene & Pseudophake \\
\hline MW & 3,62 & 3,76 & 3,21 & 3,90 \\
\hline Median & 3,62 & 3,76 & 3,14 & 3,93 \\
\hline Min & 3,33 & 3,21 & 2,63 & 3,46 \\
\hline Max & 3,98 & 4,21 & 3,95 & 4,17 \\
\hline SD & 0,19 & 0,23 & 0,33 & 0,20 \\
\hline
\end{tabular}

Tabelle 20: VKT im Gruppenvergleich, Angaben in mm 
Im Mehrfachvergleich der verschiedenen Gruppen stellte sich heraus, dass sich die VKT betreffend lediglich die Kinder nicht signifikant $(p=0,73)$ von Jugendlichen unterschieden.

Zwischen allen anderen Gruppen konnten hoch signifikante Unterschiede nachgewiesen werden. Auffällig war jedoch, dass in den Gruppen der Jugendlichen und Erwachsenen die Einzelmessungen der VKT kaum differierten ( $\mathrm{SD}=0,01$ bis $0,02 \mathrm{~mm}$ ), wohingegen bei Kindern und Pseudophaken vereinzelt auch höhere Standardabweichungen, bis hin zu 0,05 $\mathrm{mm}$ zu beobachten waren.

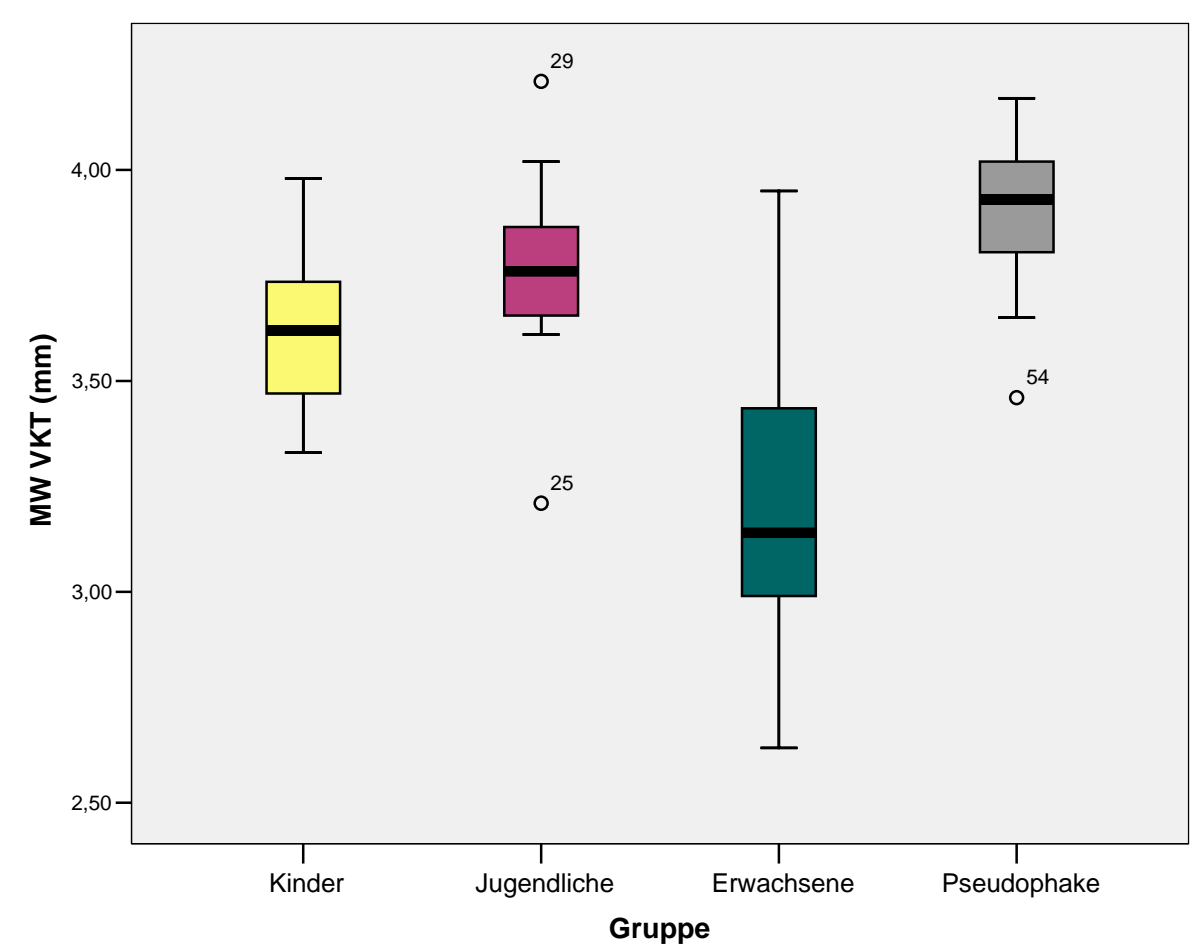

Abbildung 30: Boxplot zur Verteilung der VKT im Gruppenvergleich

\subsubsection{Augenlänge}

Wie man schon an der Verteilung der AL in Abbildung 31 erkennen kann, liegen die Mediane der AL der vier Gruppen nahe beieinander, wobei die Streuung bei Kindern im Vergleich zu den anderen drei Gruppen deutlich kleiner ausfällt.

\begin{tabular}{|l||l|l|l|l|}
\hline & Kinder & Jugendliche & Erwachsene & Pseudophake \\
\hline MW & 23,19 & 23,94 & 23,66 & 23,05 \\
\hline Median & 23,29 & 23,76 & 23,70 & 23,11 \\
\hline Min & 22,45 & 22,46 & 22,63 & 21,44 \\
\hline Max & 24,35 & 25,35 & 25,33 & 24,25 \\
\hline SD & 0,49 & 0,79 & 0,73 & 0,79 \\
\hline
\end{tabular}

Tabelle 21: AL im Gruppenvergleich, Angaben in mm 
Die Mehrfachanalyse mittels ANOVA mit dem Post-Hoc-Bonferroni-Test ergab einen signifikanten Unterschied zwischen der AL der Jugendlichen und jener der Kinder $(p=0,03)$. Zwischen der AL der Erwachsenen und der AL der Pseudophaken konnte jedoch keine signifikante Unterscheidung ermittelt werden $(\mathrm{p}=0,12)$. Ebenso wenig ergab sich zwischen der AL der Jugendlichen und jener der Erwachsenen eine signifikante Differenz $(p=1,00)$.

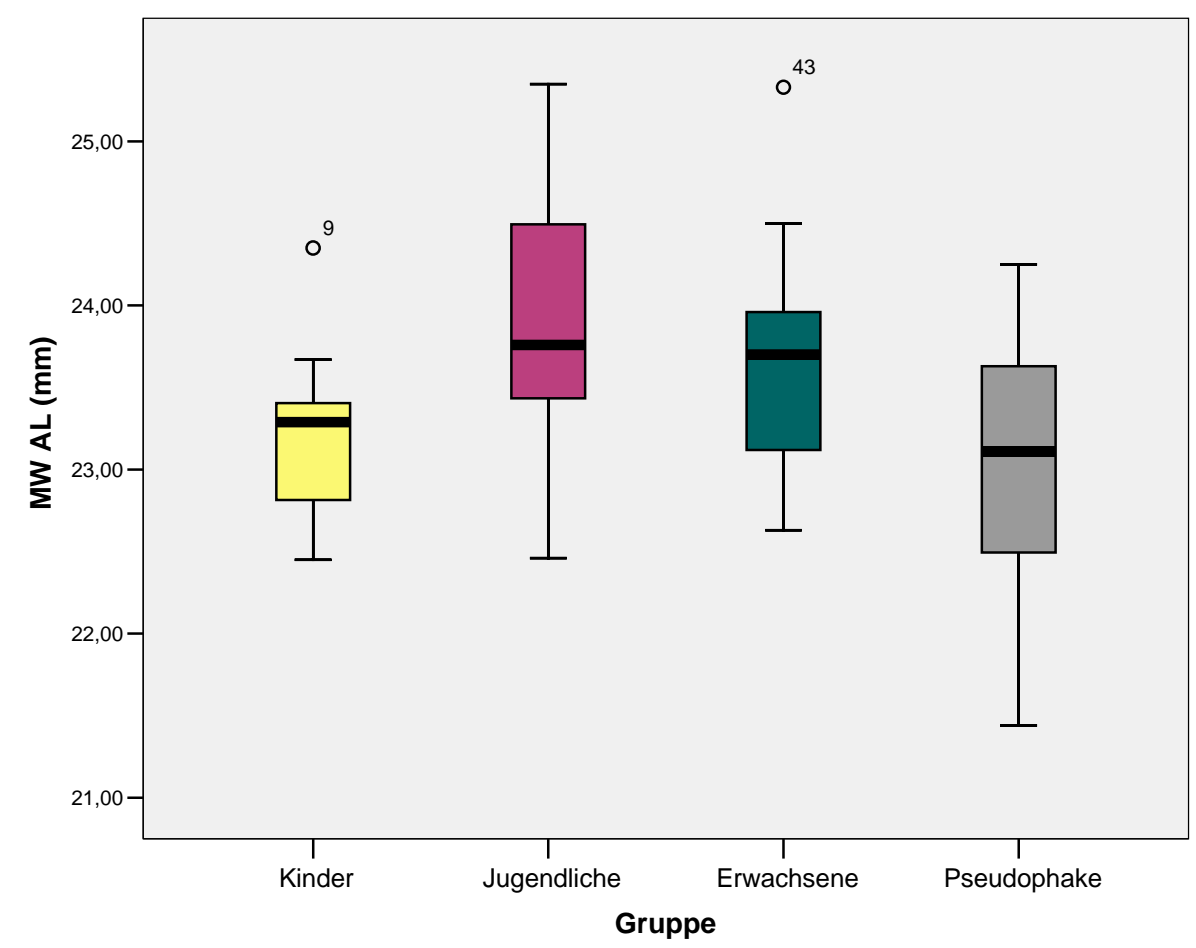

Abbildung 31: Boxplot zur Verteilung der AL im Gruppenvergleich

\subsubsection{Veränderungen während der Akkommodation}

Nun soll das Ausmaß der Akkommodationsantwort anhand der beiden gemessenen Parameter im Gruppenvergleich analysiert werden.

\subsubsection{Veränderungen der Vorderkammertiefe}

Die größten Veränderungen an der VKT bei Nahakkommodation wies die Gruppe der Jugendlichen auf. Mit Differenzen bis hin $\mathrm{zu} 0,20 \mathrm{~mm}$ verwies sie sogar die Kinder diesbezüglich auf Rang zwei.

Bei Einzelmessungen lag die Differenz der VKT bei Kindern zwar in wenigen Fällen auch im Bereich von 0,20 mm, dennoch ist die gemittelte Differenz der VKT bei Jugendlichen etwas höher als jene bei Kindern. Die Veränderung der VKT bei Nahakkommodation in der Gruppe 
der Erwachsenen fällt deutlich kleiner im Vergleich zu den jüngeren zwei Kollektiven aus. Was die Pseudophaken betrifft, so unterscheiden sie sich, wie in Abbildung 32 deutlich wird, in diesem Punkt entscheidend von den anderen Gruppen. Bei ihnen ist kaum eine Verflachung der VKT bei Nahakkommodation festzustellen. Was also die Änderungen der VKT beim Fokussieren auf nahe Zahlen betrifft, so unterscheiden sich alle Gruppen, sowohl in der Plättchen-, als auch in der Spiegel-Versuchsanordnung signifikant voneinander $(p=0,00$ bis $0,01)$.

\begin{tabular}{|l||l|l|l|l|}
\hline & Kinder & Jugendliche & Erwachsene & Pseudophake \\
\hline MW & 0,11 & 0,14 & 0,07 & 0,00 \\
\hline Median & 0,11 & 0,15 & 0,06 & 0,00 \\
\hline Min & 0,07 & 0,06 & 0,04 & 0,00 \\
\hline Max & 0,16 & 0,20 & 0,11 & 0,01 \\
\hline SD & 0,03 & 0,03 & 0,02 & 0,00 \\
\hline
\end{tabular}

Tabelle 22: Differenz VKT (unakkommodiertes - nahakkommodiertes Auge, Methode: Plättchen) im Gruppenvergleich

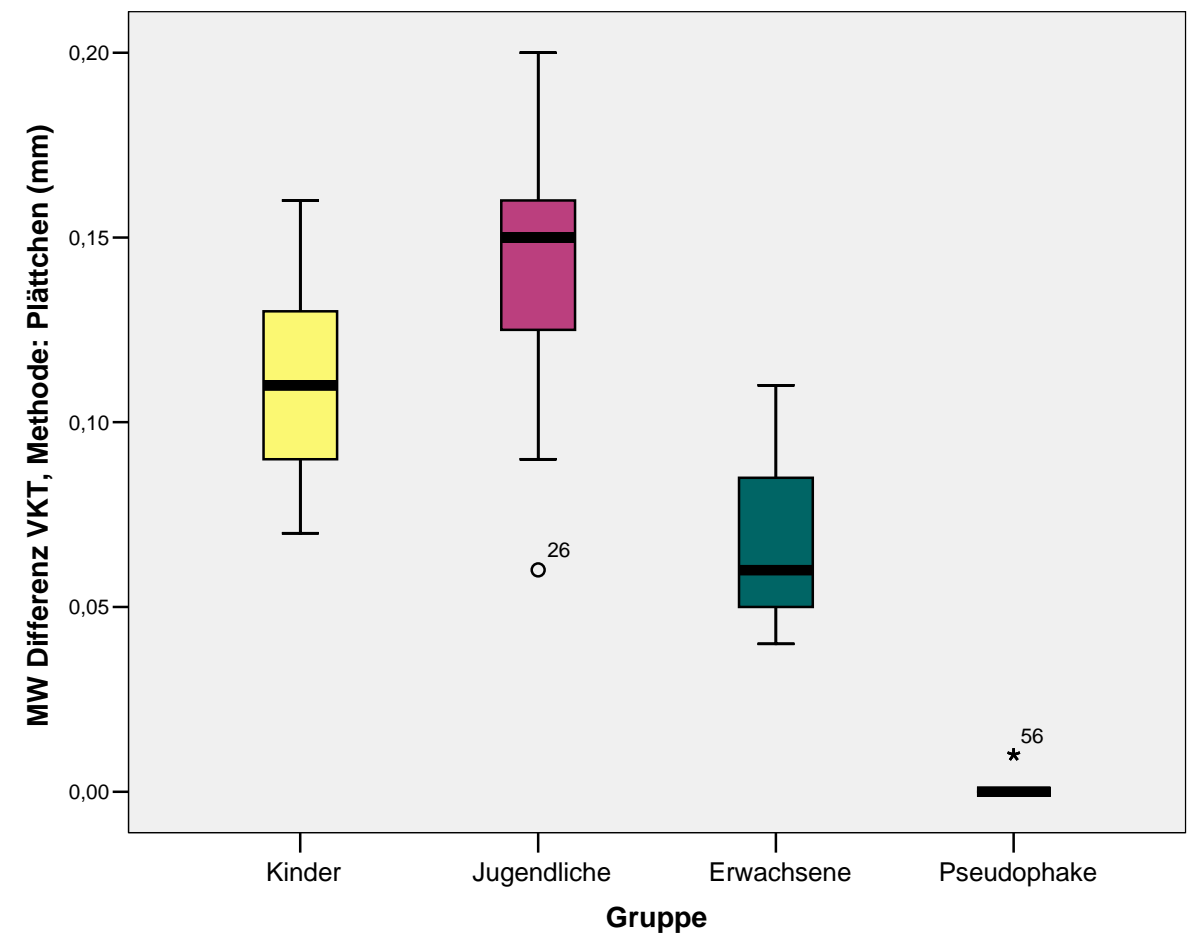

Abbildung 32: Boxplot zur Verteilung der Differenz VKT (unakkommodiertes - nahakkommodiertes Auge, Methode: Plättchen) im Gruppenvergleich 
Bei der Fernakkommodation fallen, wie bereits besprochen, die Veränderungen der VKT im Vergleich zum unstimulierten Zustand kaum auf. Daher kann hierbei auch nicht von einer signifikanten Unterscheidung zwischen den einzelnen Gruppen gesprochen werden $(p=1,00)$.

\subsubsection{Veränderungen der Augenlänge}

Was die Änderung der AL bei Nahakkommodation betrifft, so ähneln sich die Gruppen, wie aus der Tabelle 23 deutlich wird.

\begin{tabular}{|l||l|l|l|l|}
\hline & Kinder & Jugendliche & Erwachsene & Pseudophake \\
\hline MW & 0,01 & 0,01 & 0,01 & 0,01 \\
\hline Median & 0,01 & 0,01 & 0,01 & 0,01 \\
\hline Min & 0,00 & 0,00 & 0,00 & 0,00 \\
\hline Max & 0,02 & 0,04 & 0,02 & 0,01 \\
\hline SD & 0,01 & 0,01 & 0,01 & 0,01 \\
\hline
\end{tabular}

Tabelle 23: Differenz AL (nahakkommodiertes - unakkommodiertes Auge, Methode: Plättchen) im Gruppenvergleich

Die größten Einzeldifferenzen sind zwar in der Gruppe der Jugendlichen beobachtet worden, jedoch ist der Median der Differenz in allen vier Gruppen, sowohl bei den Messungen mit Plättchen als auch mit Spiegel, identisch, was in Abbildung 33 dargelegt wird.

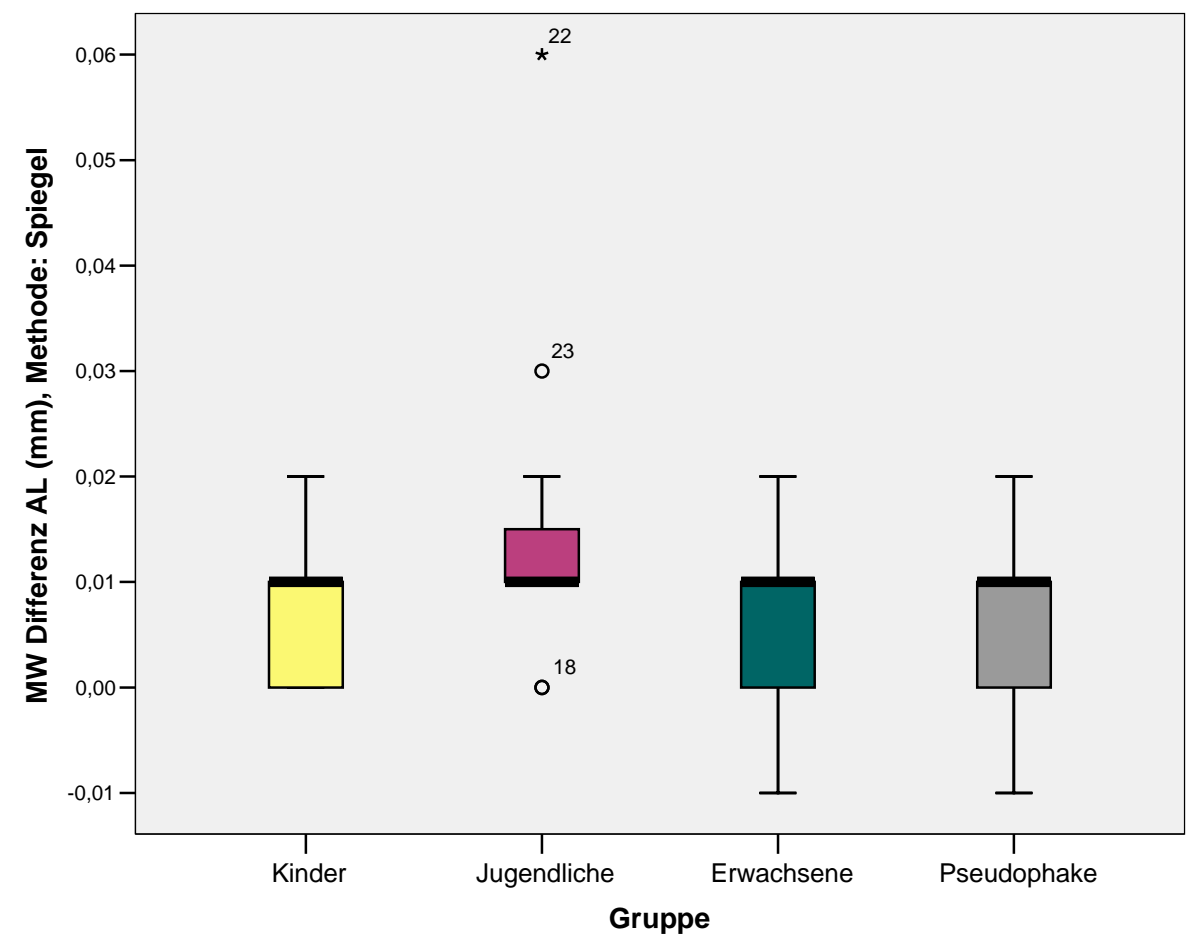

Abbildung 33: Boxplot zur Verteilung der Differenz AL (nahakkommodiertes - unakkommodiertes Auge, Methode: Spiegel) im Gruppenvergleich 
Die Änderung der AL zwischen nah- und unakkommodiertem Zustand unterscheidet sich daher auch nicht signifikant ( $\mathrm{p}=0,25$ bis 1,00) zwischen den einzelnen Gruppen.

Was die Veränderung der AL beim Blick in die Ferne betrifft, wurden ohnehin kaum Veränderungen gemessen, und wenn, so waren sowohl leichte Zunahmen als auch Abnahmen der AL dabei. Eine signifikante Unterscheidung zwischen den Gruppen konnte hierbei jedoch nicht beobachtet werden.

\subsubsection{Veränderungen in Abhängigkeit vom Alter}

An dieser Stelle wäre es interessant, einen Blick darauf zu werfen, ob sich die gemessenen Parameter und ihre Akkommodationsantwort im Laufe der Jahre verändern.

Da der Faktor Alter untersucht werden soll, werden nur gesunde Probanden verschiedener Altersstufen berücksichtigt, was mit anderen Worten heißt, dass die Gruppe der Pseudophaken aus dieser Analyse herausgenommen ist.

Bei Betrachtung der VKT fällt auf, dass diese bei Betrachtung der Kinder und Jugendlichen zunächst zunimmt. Dies wird auch in Abbildung 34 anhand der Regressionsgerade deutlich.

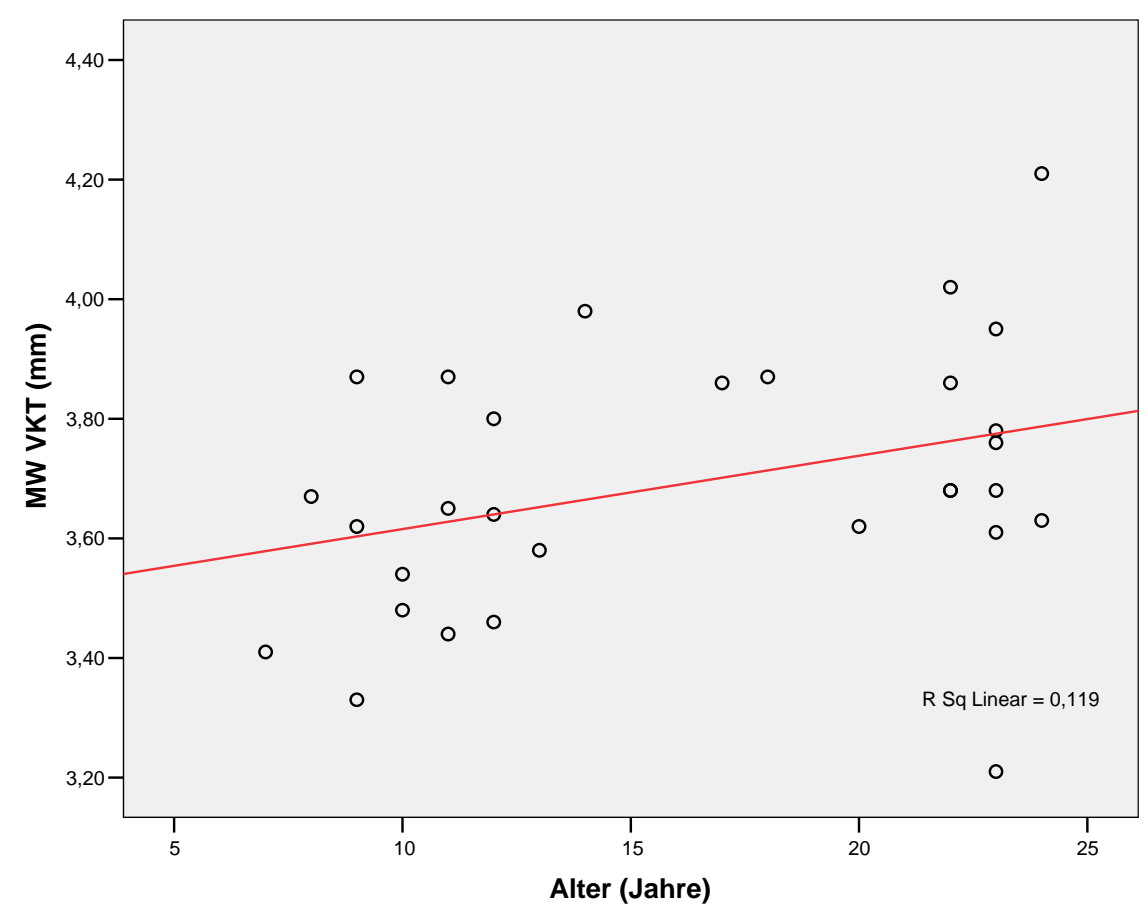

Abbildung 34: Zusammenhang Alter und VKT, Kinder und Jugendliche

Von der Jugend bis ins Alter ist jedoch eine stetige Abnahme der VKT zu beobachten, was Abbildung 35 verdeutlicht. 


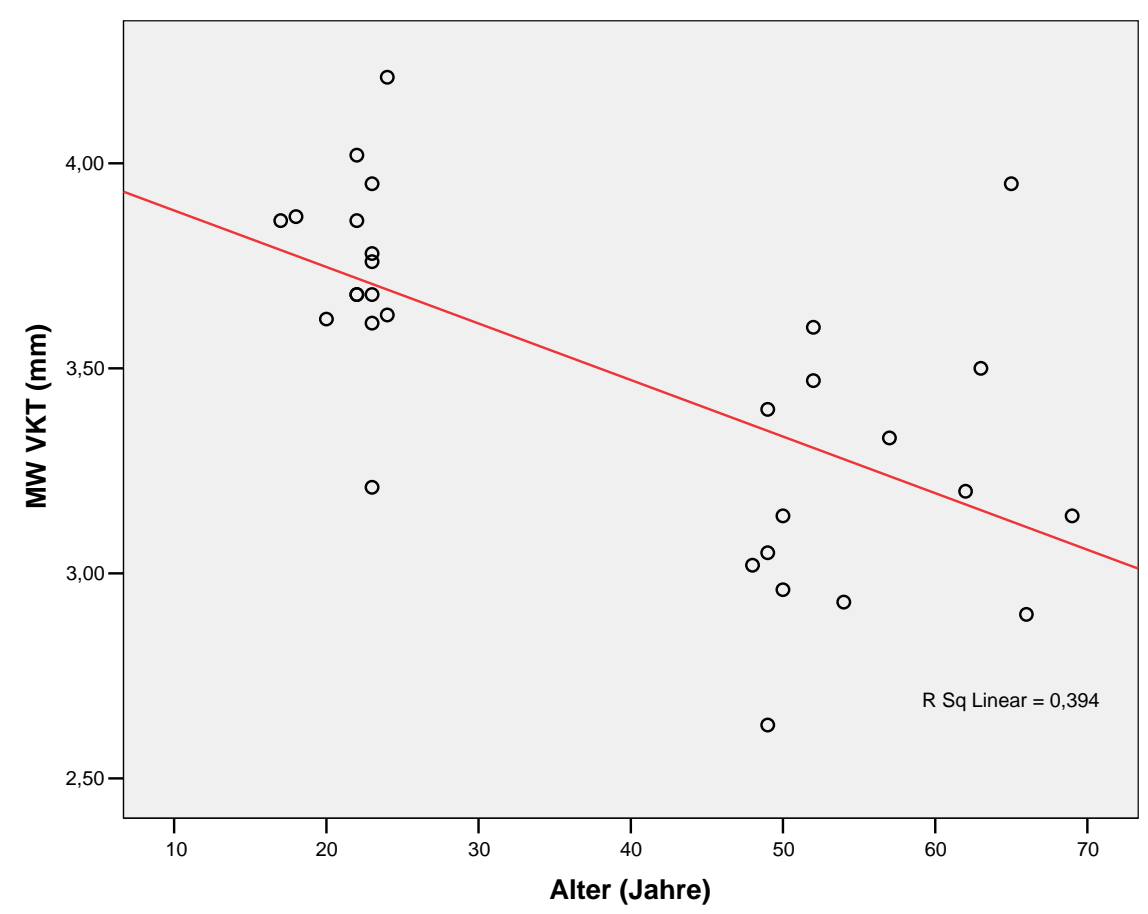

Abbildung 35: Zusammenhang Alter und VKT, Jugendliche und Erwachsene

Auch die Akkommodationsantwort, gemessen in der Differenz der VKT (unakkommodiertes nahakkommodiertes Auge) nimmt mit dem Alter deutlich ab.

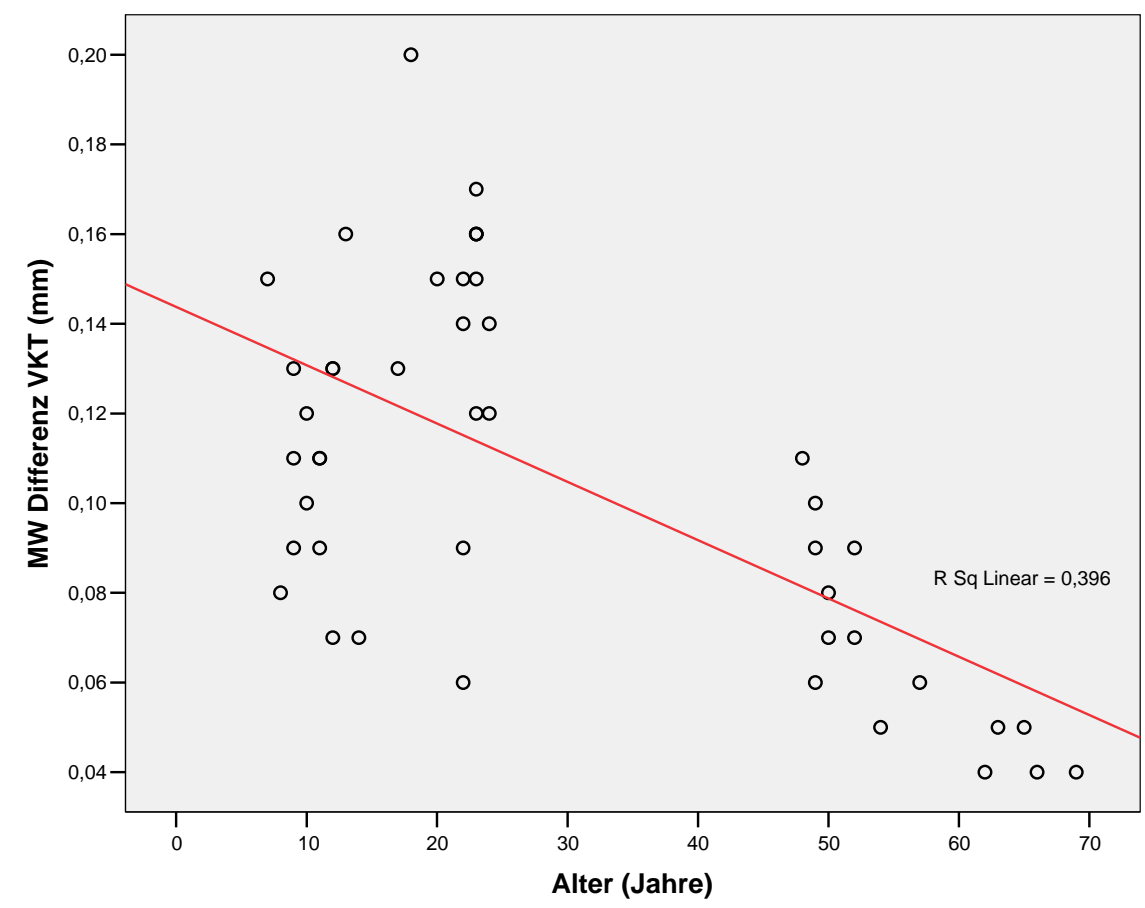

Abbildung 36: Zusammenhang Alter und Akkommodationsantwort, gemessen in der Differenz der VKT(mm) 
Bei gemittelten Differenzen der VKT findet man bei Erwachsenen, wie in Abbildung $36 \mathrm{zu}$ sehen ist, als Minimum einen Wert von 0,04 mm. Wenn man jedoch einen Blick auf die individuellen Einzelmessungen wirft, sind auch kleinere Differenzen, bis hin zu 0,00 mm in wenigen Einzelfällen bei Erwachsenen beobachtet worden.

Was die AL betrifft, so ändert sich diese nicht signifikant mit dem Alter. Das wird auch anhand der kaum vorhandenen Steigung der Regressionsgerade in Abbildung 37 deutlich.

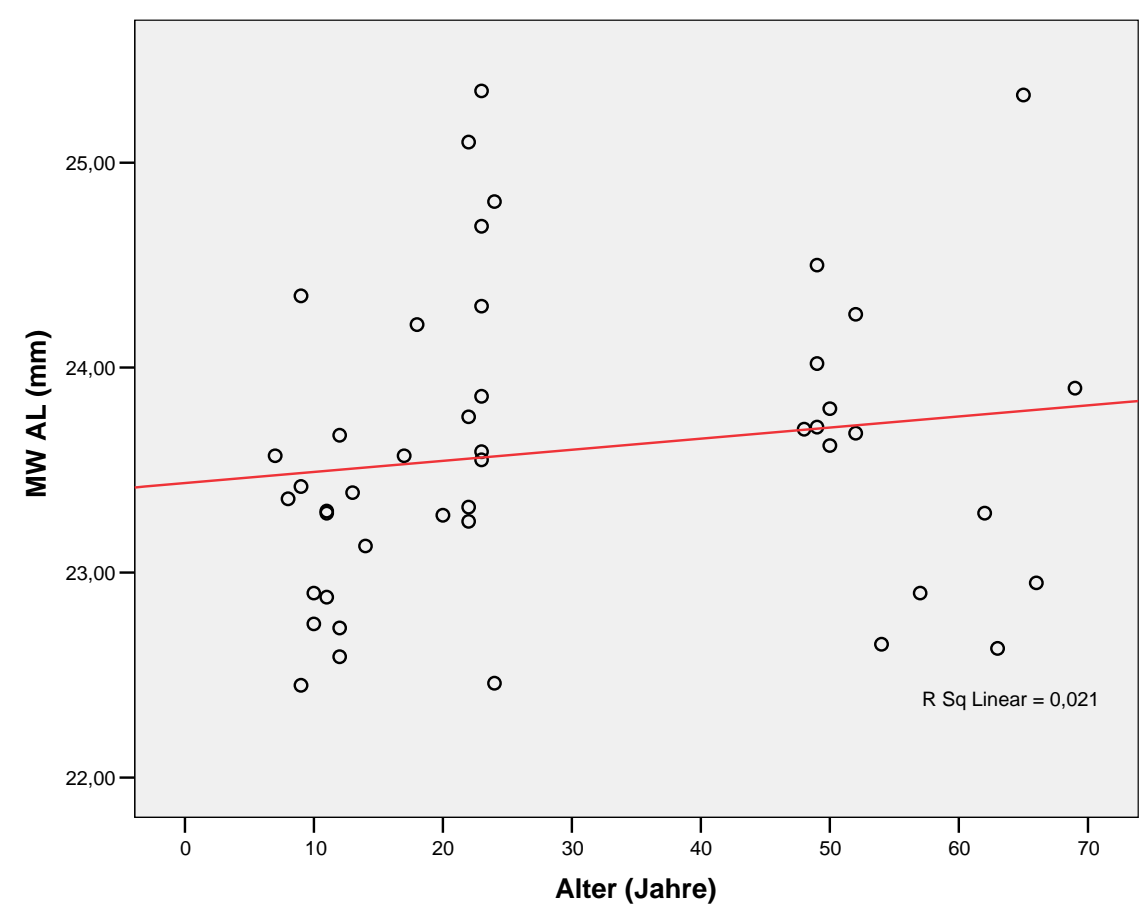

Abbildung 37: Zusammenhang Alter und AL

Sowohl bei Kindern und Jugendlichen als auch bei Erwachsenen findet man kürzere und längere Achslängen der Augen. Die Extremwerte der Jugendlichen und jene der Erwachsenen sind was AL betrifft, nahezu identisch (Min: 22,46 bis 22,63 mm; Max: 25,33 bis 25,35 mm).

\subsection{Methodenvergleich}

Die Werte der Messungen in den beiden Versuchsreihen Plättchen und Spiegel, werden nun daraufhin geprüft, ob zwischen ihnen eine signifikante Korrelation vorhanden ist. In den Grafiken soll die Bland-Altman-Auftragung zum Einsatz kommen, eine statistische Methode, mit der Messtechniken verglichen werden können. Hierbei wird die Differenz der beiden Methoden auf der Y-gegen deren Mittelwerte auf der X-Achse aufgetragen. Eine horizontale 
Linie zeigt die gemittelte Differenz der beiden Methoden an. Falls diese innerhalb des Konfidenzintervalls von $\pm 1,96$ (SD) liegt, können die Methoden, solange das Signifikanzniveau von 0,05 als klinisch ausreichend betrachtet wird, gleichwertig verwendet werden.

\subsubsection{Messungen der Vorderkammertiefe}

Wie man in Abbildung 38 gut sehen kann, liegt die gemittelte Differenz zwischen den beiden Methoden auf der Nulllinie, woraus man schließen kann, dass es kaum einen Unterschied zwischen den beiden Messtechniken bei der Erfassung der VKT während Akkommodation gibt. Lediglich drei der sechzig verglichenen Messungen (5\%) liegen nicht innerhalb des 95\%-Konfidenzintervalls.

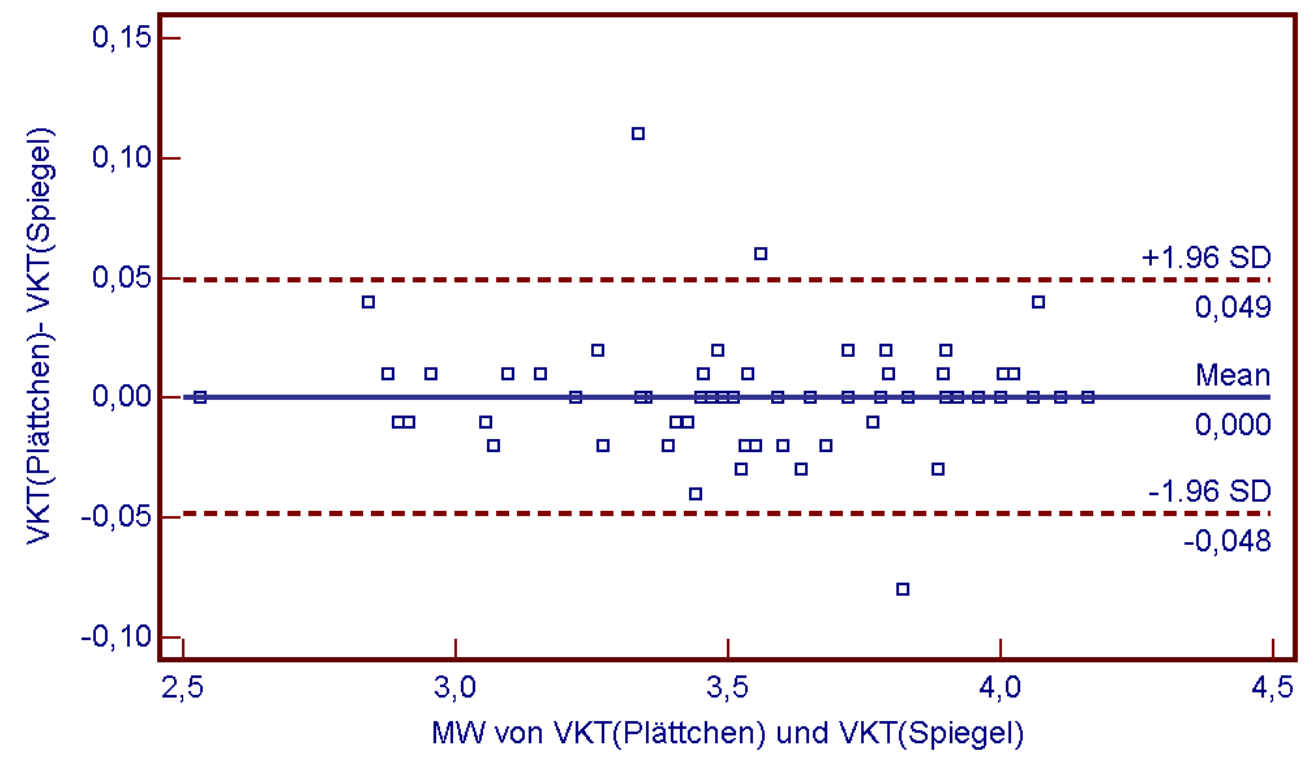

Abbildung 38: Änderung der VKT bei Nahakkommodation (Angaben in mm) im Methodenvergleich

Die Korrelation zwischen den Messungen der VKT mit den beiden Methoden erwies sich als hoch und signifikant, wie in Tabelle 24 dargelegt wird. Am schwächsten ist diese Korrelation bei Messungen der VKT während Nahakkommodation in der Kindergruppe ausgeprägt, aber selbst hier liegt sie auf einem sehr hohen Niveau, nämlich bei 0,98.

Die Veränderung der VKT während der Akkommodation im Vergleich zwischen Plättchen und Spiegel wird zunächst anhand der deskriptiven Statistik in Tabelle 25 deutlich. Die Korrelation der beiden Messmethoden, was die Differenz der VKT bei Nahakkommodation betrifft, ist hoch $(\mathrm{r}=0,905)$. 


\begin{tabular}{|l||l|l|}
\hline & Korrelation & Signifikanz \\
\hline $\begin{array}{l}\text { MW VKT bei } \\
\text { Nahakkommodation }\end{array}$ & 0,998 & 0,00 \\
\hline $\begin{array}{l}\text { MW VKT bei } \\
\text { Fernakkommodation }\end{array}$ & 1,00 & 0,00 \\
$\begin{array}{l}\text { MW Differenz VKT } \\
\text { (unakkommodiertes- } \\
\text { akkommodiertes } \\
\text { Auge) }\end{array}$ & 0,905 & 0,00 \\
\hline
\end{tabular}

Tabelle 24: Korrelation zwischen den Methoden bei Messung der VKT

\begin{tabular}{|l||l|l|}
\hline & Plättchen & Spiegel \\
\hline MW & 0,14 & 0,14 \\
\hline Median & 0,15 & 0,15 \\
\hline Min & 0,06 & 0,07 \\
\hline Max & 0,20 & 0,18 \\
\hline SD & 0,03 & 0,02 \\
\hline
\end{tabular}

Tabelle 25: Differenz VKT (unakkommodiertes - nahakkommodiertes Auge) im Methodenvergleich, Angaben in mm

Im t- Test für gepaarte Stichproben ergeben sich weder für Messungen der VKT bei Nahnoch bei Fernakkommodation signifikante Unterschiede zwischen den beiden Methoden $(\mathrm{p}=0,90$ bis 0,92$)$.

Auch für die gemittelte Differenz der VKT bei Messungen mit beiden Methoden liegt die Ausgleichslinie genau auf der Nullgeraden, was in diesem Zusammenhang für eine hohe Vergleichbarkeit der beiden Messtechniken spricht (s. Abbildung 39).

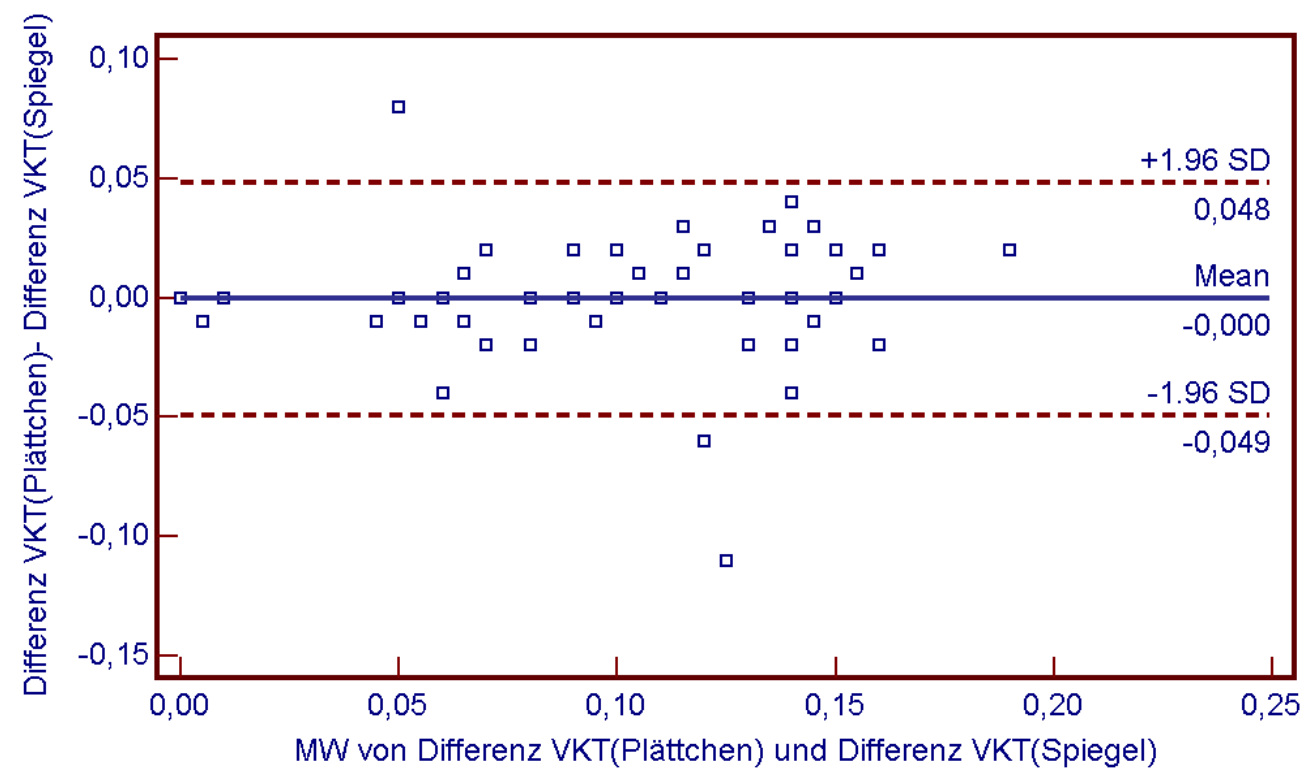

Abbildung 39: Differenz VKT (unakkommodiertes - nahakkommodiertes Auge, Angaben in mm) im Methodenvergleich 


\subsubsection{Messungen der Augenlänge}

Bei Betrachtung der in Tabelle 26 dargelegten Korrelationen zwischen den Messungen der AL bei Nah- und Fernakkommodation im Vergleich der beiden Methoden, überrascht es nicht, dass sich auch im t- Test keine signifikante Unterscheidung zwischen Plättchen und Spiegel ergab ( $\mathrm{p}=0,20$ bis 0,32$)$.

\begin{tabular}{|l||l|l|}
\hline & Korrelation & Signifikanz \\
\hline $\begin{array}{l}\text { MW AL bei } \\
\text { Nahakkommodation }\end{array}$ & 1,00 & 0,00 \\
$\begin{array}{l}\text { MW AL bei } \\
\text { Fernakkommodation }\end{array}$ & 1,00 & 0,00 \\
\hline $\begin{array}{l}\text { MW Differenz AL } \\
\text { (nahakkommodiertes- } \\
\text { unakkommodiertes } \\
\text { Auge) }\end{array}$ & 0,39 & 0,00 \\
\hline
\end{tabular}

Tabelle 26: Korrelation zwischen den Methoden bei Messung der AL

Die Ausgleichsgerade im Bland- Altman- Diagramm (Abbildung 40) liegt hier zwar leicht unterhalb der Nullgeraden, dennoch befinden sich 57 der 60 (95\%) in den Vergleich einbezogenen Mittelwerte der AL- Messungen bei Nahakkommodation innerhalb des 95\%Konfidenzintervalls.

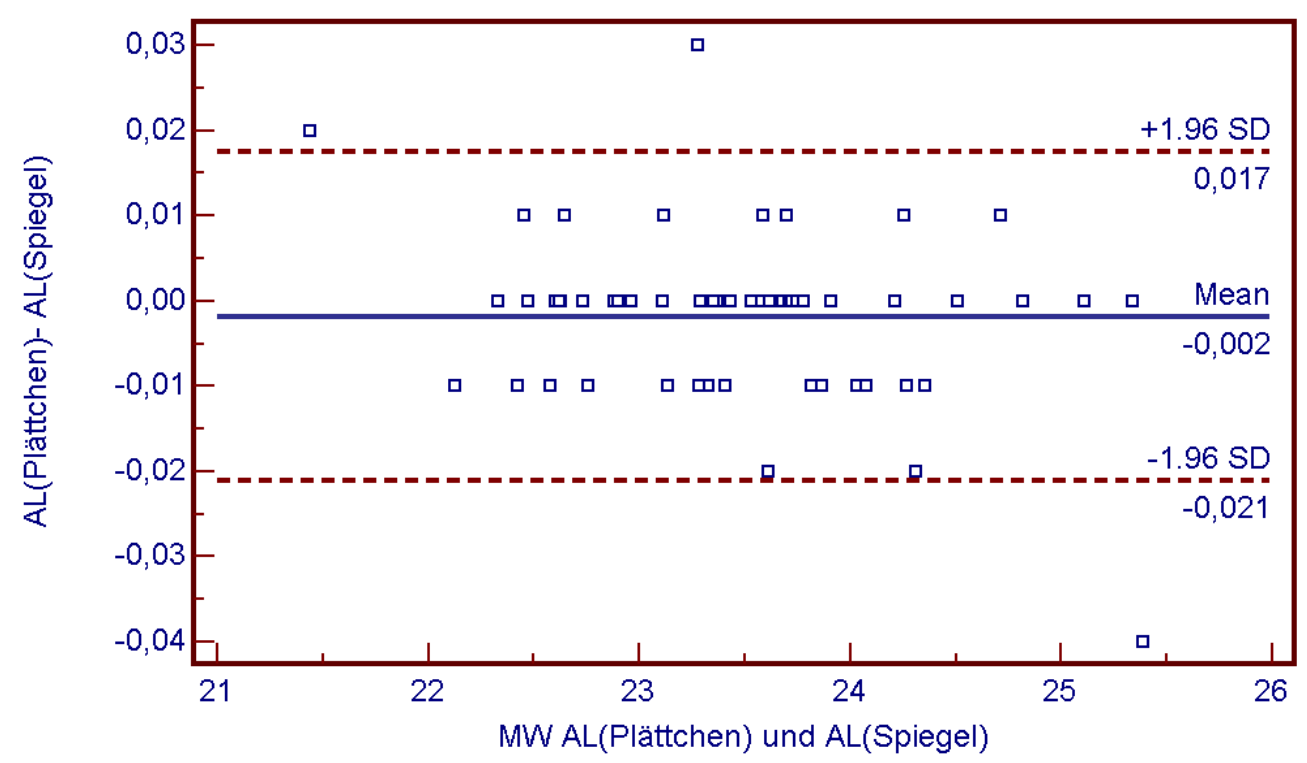

Abbildung 40: AL bei Nahakkommodation (Angaben in mm) im Methodenvergleich

Wie man in Tabelle 27 erkennen kann, unterschieden sich die beiden Methoden in den Ergebnissen zur Differenz der AL (nahakkommodiertes- unakkommodiertes Auge) nicht voneinander. Dennoch ist in diesem Punkt ihre Korrelation zwar signifikant, jedoch niedrig 
$(\mathrm{r}=0,393)$. Auffällig ist lediglich, dass in der Messreihe mit Spiegel eine höhere Verlängerung der AL bei einer jugendlichen Testperson ermittelt wurde. Bei jeweils einem erwachsenen und einem pseudophaken Probanden wurde eine Verkürzung der AL von 0,01 $\mathrm{mm}$ bei Nahakkommodation und Spiegel- Methode gemessen. Dadurch ergibt sich beim Vergleich der beiden Methoden eine etwas niedrigere, aber dennoch signifikante Korrelation $(r=0,393)$ bei der errechneten Differenz der AL (nahakommodiertes - unakkommodiertes Auge).

\begin{tabular}{|l||l|l|}
\hline & Plättchen & Spiegel \\
\hline MW & 0,01 & 0,01 \\
\hline Median & 0,01 & 0,01 \\
\hline Min & 0,00 & 0,00 \\
\hline Max & 0,04 & 0,06 \\
\hline SD & 0,01 & 0,01 \\
\hline
\end{tabular}

Tabelle 27: Differenz AL (nahakkommodiertes- unakkommodiertes rechtes Auge) im Methodenvergleich bei Jugendlichen, Angaben in $\mathbf{m m}$

\subsection{Untersuchervergleich}

Zum Schluss soll noch darauf eingegangen werden, ob die Messungen vom Untersucher abhängig waren.

\subsubsection{Vorderkammertiefe}

Zunächst wird wieder einmal die VKT betrachtet. Wie bereits aus Tabelle 28 ersichtlich wird, differierten die Mittelwerte der Messungen der VKT während Nahakkommodation zwischen den beiden Untersuchern höchstens um 0,02 mm.

\begin{tabular}{|c|c|c|c|c|}
\hline & \multicolumn{4}{|c|}{ MW VKT bei Nahakkommodation (mm) } \\
\hline & \multicolumn{2}{|c|}{ Messung mit Plättchen } & \multicolumn{2}{|c|}{ Messung mit Spiegel } \\
\hline & Untersucher 1 & Untersucher 2 & Untersucher 1 & Untersucher 2 \\
\hline Proband 1 & 3,52 & 3,52 & 3,54 & 3,52 \\
\hline Proband 2 & 3,62 & 3,61 & 3,59 & 3,59 \\
\hline Proband 3 & 3,54 & 3,55 & 3,52 & 3,51 \\
\hline Proband 4 & 3,49 & 3,49 & 3,52 & 3,52 \\
\hline Proband 5 & 3,47 & 3,47 & 3,46 & 3,46 \\
\hline
\end{tabular}

Tabelle 28: Untersuchervergleich bei Anwendung zweier Methoden 


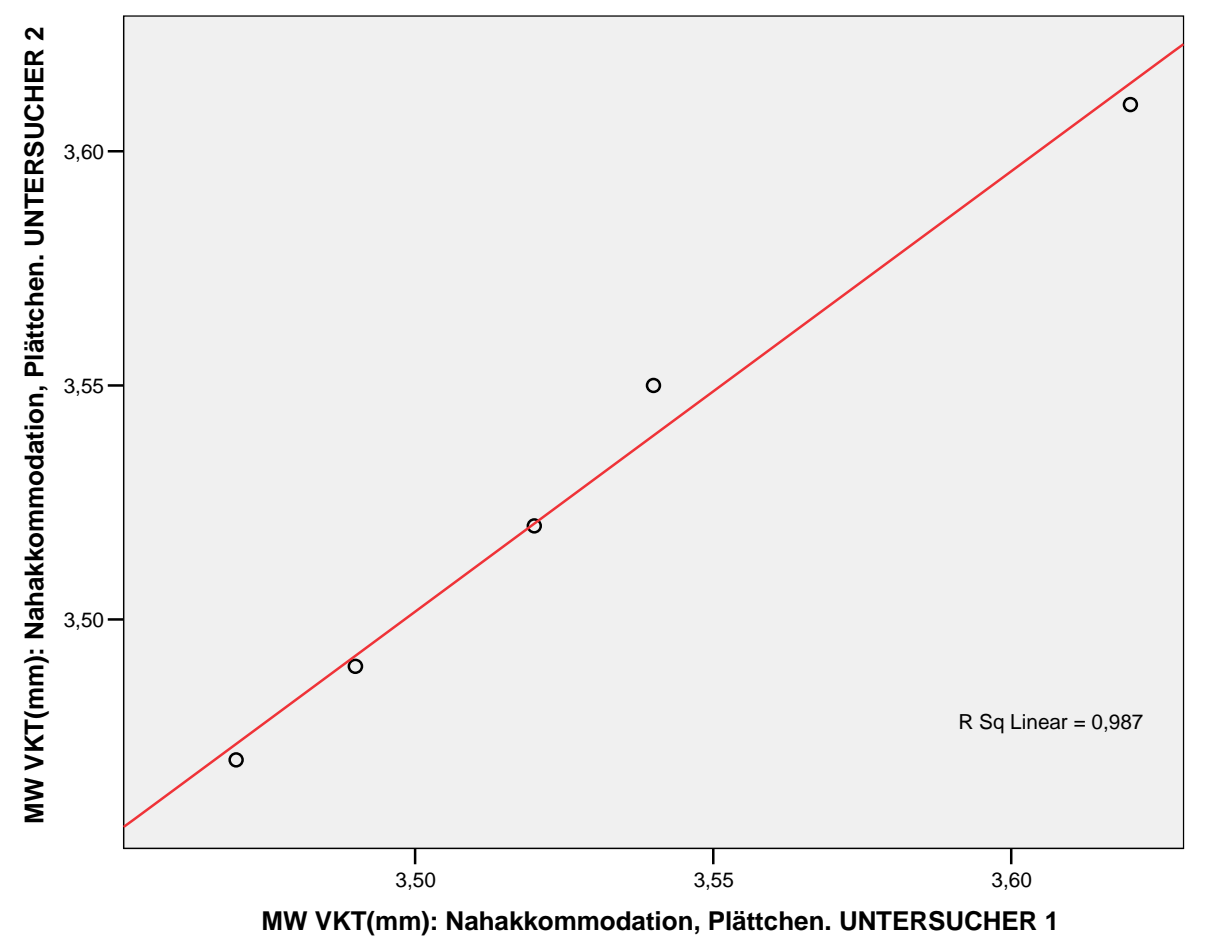

Abbildung 41: VKT bei Nahakkommodation im Untersuchervergleich

Bei 60\% der Messungen ergab sich jedoch gar kein Unterschied bei Mittelwerten der VKT bei Nahakkommodation. Die in Abbildung 41, in der die von dem einen Untersucher gemessenen VKT gegen jene aufgetragen wurden, die vom zweiten Untersucher beobachtet wurden, eingezeichnete Regressionsgerade hat annähernd eine Steigung von 1. Dies spricht für eine hohe Ähnlichkeit der gemessenen Werte.

\begin{tabular}{|c|c|c|}
\hline & Korrelation & Signifikanz \\
\hline $\begin{array}{l}\text { MW VKT bei } \\
\text { Nahakkommodation, } \\
\text { Methode: } \\
\text { PLÄTTCHEN }\end{array}$ & 0,994 & 0,001 \\
\hline $\begin{array}{l}\text { MW VKT bei } \\
\text { Nahakkommodation, } \\
\text { Methode: SPIEGEL }\end{array}$ & 0,982 & 0,003 \\
\hline
\end{tabular}

Tabelle 29: Korrelation zwischen den Untersuchern bei Messung der VKT

In der statistischen Analyse ergab sich ebenfalls eine signifikant hohe Korrelation der Messungen der VKT zwischen den beiden Untersuchern bei Verwendung beider Methoden. Der Zusammenhang wird in Tabelle 29 dargelegt. 


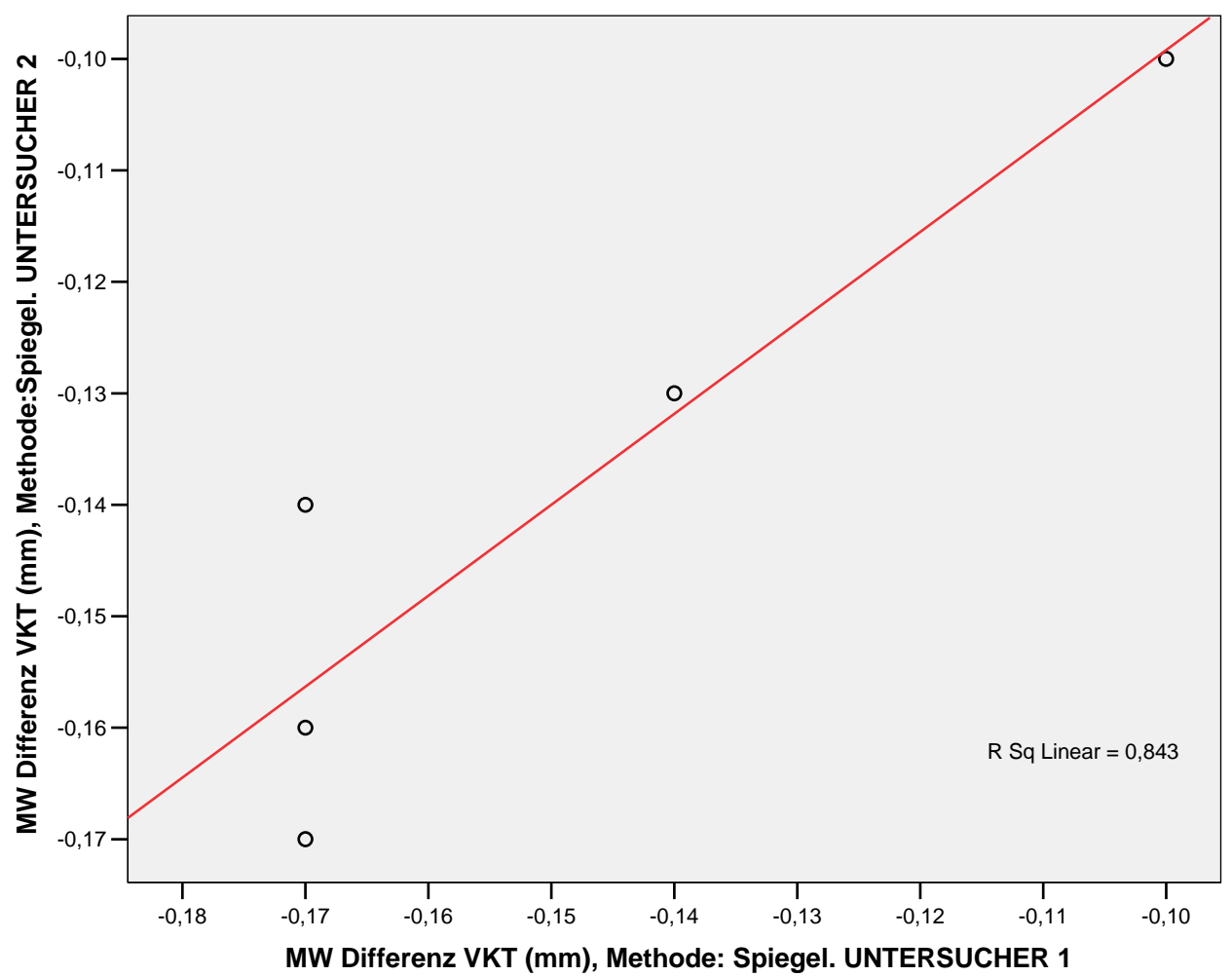

Abbildung 42: Diferenz VKT(unakkommodiertes - nahakkommodiertes Auge) im Untersuchervergleich

Entsprechend unterschieden sich auch die errechneten Differenzen der VKT (nahakkommodiertes - unakkommodiertes Auge) bei beiden Untersuchern kaum voneinander. Der Zusammenhang wird in Abbildung 42 dargelegt.

\subsubsection{Augenlänge}

Was die AL betrifft, so war die Korrelation zwischen den Untersuchern noch höher, nämlich $\mathrm{r}=1,00$. Die gemittelten Ergebnisse der Messungen der beiden Untersuchern ergaben exakt gleiche Werte.

\begin{tabular}{|c|c|c|}
\hline & Korrelation & Signifikanz \\
\hline $\begin{array}{l}\text { MW AL bei } \\
\text { Nahakkommodation, } \\
\text { Methode: } \\
\text { PLÄTTCHEN }\end{array}$ & $\mid 1,00$ & 0,00 \\
\hline $\begin{array}{l}\text { MW AL bei } \\
\text { Nahakkommodation, } \\
\text { Methode: SPIEGEL }\end{array}$ & $\mid 1,00$ & 0,00 \\
\hline
\end{tabular}

Tabelle 30: Korrelation zwischen den Untersuchern bei Messung der AL 


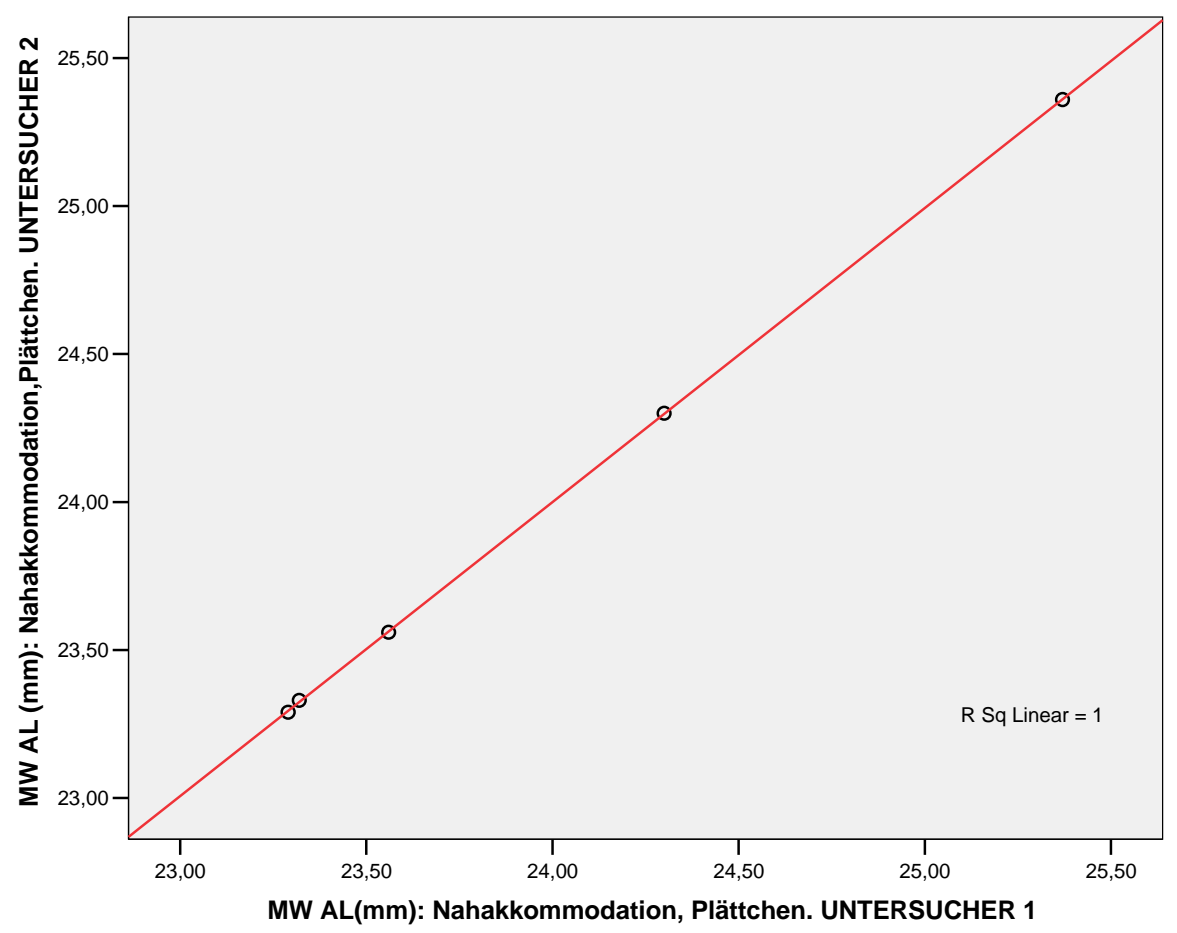

Abbildung 43: AL bei Nahakkommodation, Methode: Plättchen, im Untersuchervergleich

Die hohe Korrelation der Ergebnisse der beiden Untersucher bei den Messungen der AL konnte bei Verwendung beider Methoden, also bei Plättchen und Spiegel, beobachtet werden. Dieses wird in Abbildung 43 und 44 deutlich. In beiden Fällen hat die eingezeichnete Regressionsgerade eine Steigung von 1, was dafür spricht, dass sich bei den zwei Untersuchern als Mittelwert der Einzelmessungen gleiche Werte für die AL ergeben haben.

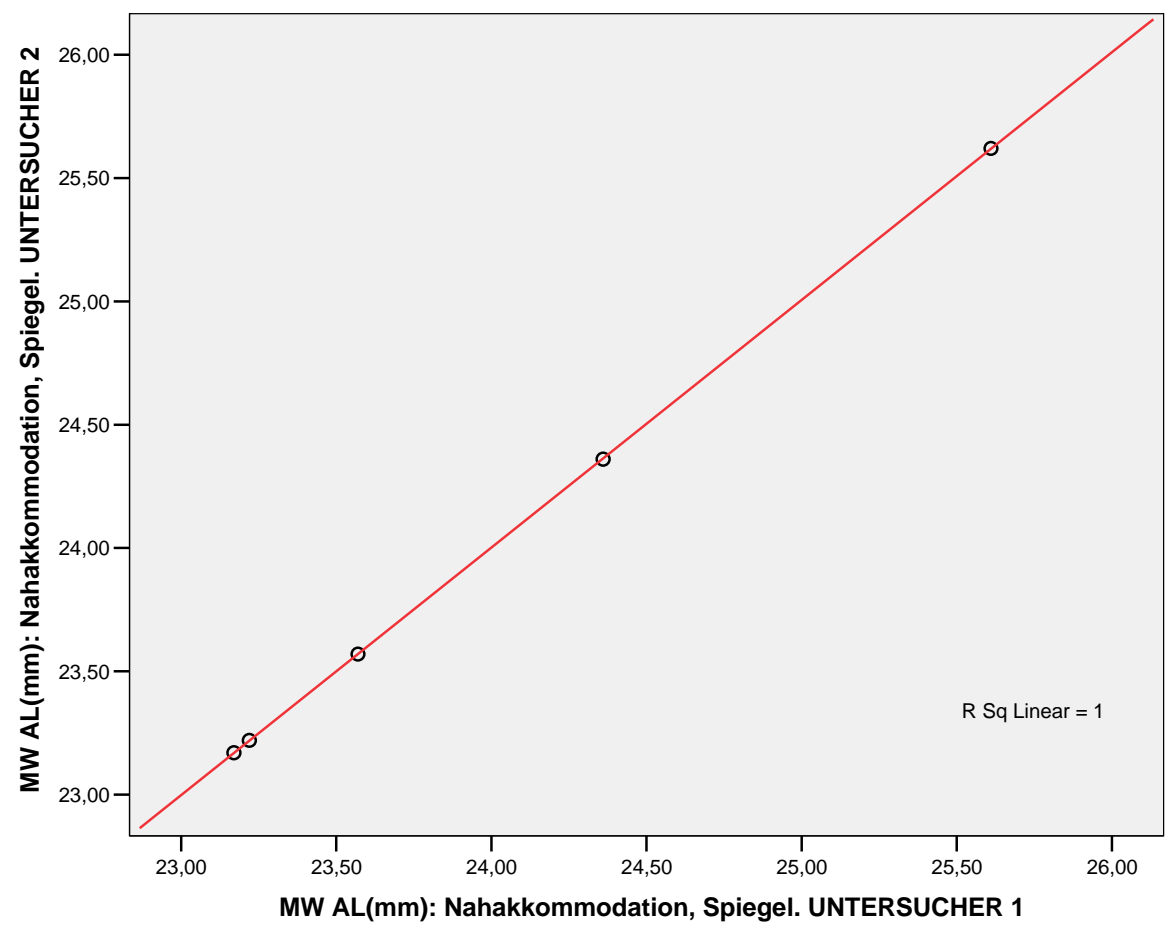

Abbildung 44: AL bei Nahakkommodation, Methode: Spiegel, im Untersuchervergleich 


\section{Diskussion}

Das Verständnis der Akkommodation, ihrer Auswirkungen am Auge und ihren Veränderungen im Alter waren Gegenstand zahlreicher Publikationen der letzten Jahre.

Bei Eingabe der Wörter „accommodation“ und „eye“ in der Pubmed- Suchleiste, erscheinen zur Zeit auf Anhieb 2654 Artikel und das sind mit Sicherheit nicht alle, die zu dem Thema vorhanden sind. Mit dem Bestreben, Akkommodation auch im Alter möglich zu machen, wurde es immer wichtiger, die anatomischen Strukturen und die Rolle, die sie bei der Nahund Ferneinstellung des Auges spielen, genau zu untersuchen.

\subsection{Stimulation und Erfassung der Akkommodation}

\subsubsection{Methoden zur Erfassung der Akkommodation}

Die Methoden und Geräte, die dabei verwendet wurden, unterschieden sich jedoch erheblich voneinander. Neben Ansätzen, die anhand mathematischer Formeln die Werte der Augenparameter herzuleiten versuchten (18, S.445; 61, S.200), gab es natürlich auch verschiedene Messtechniken, die hierbei zum Einsatz kamen. Während zur Erfassung der Brechkraftänderung häufig die Refraktometrie oder die Skiaskopie herangezogen wurden, kam bei der Messung der tatsächlichen Änderungen der Augenparameter während der Akkommodation in der Vergangenheit neben der Scheimpflug-Fotografie (49, S.569) vorwiegend die Ultraschalluntersuchung zum Einsatz, die seit 1956 in der Augenheilkunde verwendet wird. (68, S.488) Physiologische Stimulation der Akkommodation erfolgte durch Anbringen von Optotypen in unterschiedlichen Abständen (7, S.524) oder durch Vorsetzen von Minusgläsern vor eines der Augen. (29, S.13) Am jeweils anderen Auge wurde in den mit Ultraschall durchgeführten Studien letztlich die Akkommodationsantwort gemessen. Vom Nachteil hierbei war sicherlich, dass diese Methode nicht ohne den Einsatz von Lokalanästhetika und den direkten Kontakt zum Auge, der die Gefahr von kornaelen Infektionen birgt, auskam.

Daher gab es bereits in den sechziger Jahren Bestrebungen, nicht-invasive Methoden zur Erfassung der Augenparameter zu verwenden. Diese bekamen 1986 mit der Entwicklung der 
PCI (partielle Kohärenz-Interferometrie) einen großen Schub. Zunächst schien die PCI gegenüber longitudinalen Augenbewegungen anfällig zu sein, doch dieses Problem konnte mit der Einführung der Doppelstrahltechnik, die auch im IOL-Master bei der Messung der AL verwendet wird, behoben werden. (19, S.2789)

Seither wurde diese Methode in zahlreichen Studien untersucht.

Sie wurde in Bezug auf die Erfassung der VKT und der Linsendicke für präziser als die Ultraschalltechnik befunden (20, S.1304), welcher sie bei Messungen der AL ebenfalls deutlich überlegen ist. (21, S.2141) Die Genauigkeit der PCI bei der Bestimmung der AL wurde anfangs bei $25 \mu \mathrm{m}$ angegeben (37, S.616), inzwischen jedoch geht man von einer Präzision zwischen 0,3 und $10 \mu \mathrm{m}$ aus. (21, S.2141) Im Vergleich dazu wurde bei Verwendung eines 10 MHz-Schallkopfs von einer Genauigkeit in der Größenordnung von 100 bis $120 \mu \mathrm{m}$ berichtet. (103, S.1962) Selbst in der Gruppe der Pseudophaken erwies sich PCI um den Faktor 20 besser als die konventionelle Ultraschalltechnik und ermöglichte Messungen der AL mit einer Genauigkeit von $5 \mu \mathrm{m}$. (24, S.1087)

Der IOL-Master verwendet, wie bereits in Kapitel 2 erörtert wurde, die PCI-Methode bei der Messung der AL. Für die Erfassung der VKT greift er auf die gängige Spaltlampentechnik zurück. Diese Arbeit hatte zum Ziel, kleinste Veränderungen am Auge während der Akkommodation zu erfassen, daher war es besonders wichtig, ein Gerät mit hoher Auflösung und Präzision einzusetzen. Da darüber hinaus an der Studie neben den gängigen Gruppen der emmetropen Jugendlichen und Erwachsenen, auch Kinder und Pseudophake teilnahmen, war es entscheidend, dass das verwendete Gerät auch in diesen Kollektiven zuverlässige Ergebnisse liefert. Diese Kriterien treffen auf den IOL-Master zu. In den letzten Jahren wurden die Messungen mit dem Gerät vielfach als genau und gut reproduzierbar beurteilt. (16, S.235; 87, S.458; 71, S.85) Auch bei Messungen an Kindern wurde bezweifelt, dass die Ultraschalltechnik eine ausreichende Genauigkeit zur Erfassung von Veränderungen während der Akkommodation bietet (55, S.35), und der IOL-Master in Publikationen als Standard für Ermittlung der AL und VKT bei Kindern empfohlen. (11, S.829) Damit brachte der IOLMaster ideale Voraussetzungen für die Verwendung in dieser Arbeit.

Inzwischen wurde eine weitere Technik zur statischen und dynamischen Analyse des vorderen Augenabschnitts eingeführt. Die so genannte optische Kohärenz-Tomographie verwendet Licht von $1310 \mathrm{~nm}$ Wellenlänge um die Vorderkammer abzubilden. Sie ermöglicht eine Aussage über den Durchmesser und die Tiefe der Vorderkammer, die Krümmungsradien 
der Linse, die Linsendicke und den iridokornealen Winkel. Durch die Tatsache, dass sie widerum keines direkten Kontaktes zum Auge bedarf und eine hohe Auflösung von $10 \mu \mathrm{m}$ bietet, wird sie die Aussagemöglichkeiten zu Veränderungen während der Akkommodation in Zukunft vermutlich noch erweitern. Bislang wurde sie jedoch nur in einer kleinen Minderheit von Studien angewendet. $(5$, S.1835)

\subsubsection{Reproduzierbarkeit und Vergleich der verwendeten Methoden}

Aus Publikationen anderer Arbeitsgruppen ist bereits bekannt, dass der IOL-Master untersucherunabhängige Ergebnisse liefert. (45, S.518) Mit Variationskoeffizienten von $0,09 \%$ bei der AL und $0,82 \%$ bei der VKT wurde die gute Reproduzierbarkeit der durch verschiedene Untersucher durchgeführten Messungen mit dem IOL-Master in anderen Studien belegt. (103, S.1965) Auch die Erfahrung des Untersuchers im Umgang mit dem IOL-Master spielt keine große Rolle für die Genauigkeit der Messungen. (93, S.32) Mit diesem also verlässlichen Mittel ließ es sich gut überprüfen ob die Ergebnisse der angewendeten Messmethoden (Plättchen und Spiegel) mit dem Untersucher variierten. Dabei führten wie beschrieben eine erfahrene Augenschwester und ich bestimmte Messungen an fünf Probanden durch. Bei der AL waren sehr hohe Korrelationen $(r=1,0)$ der Mittelwerte sowohl bei Nahakkommodation mit Plättchen als auch mit Spiegel festzustellen. Was die Mittelwerte der VKT bei Naheinstellung betrifft, so ließen sich signifikant hohe Korrelationen im Bereich von $r=0,99$ mit Plättchen, bzw. $r=0,98$ mit Spiegel im Interuntersuchervergleich feststellen. Das ist ein überzeugender Beweis dafür, dass sich die hierbei angewendeten Methoden unabhängig vom Untersucher gut einsetzen lassen, sowie ein gut reproduzierbares physiologisches Mittel zur Stimulation der Akkommodation darstellen.

Bei beiden Messreihen wurde in dieser Arbeit die binokulare Akkommodationsleistung beurteilt, da keines der Augen abgedeckt wurde. Das bedeutet, dass sowohl Akkommodation als auch Konvergenz als Auslöser für die Änderungen in Betracht zu ziehen sind (6, S.304), worauf später noch genauer eingegangen wird.

Interessant ist jedoch, dass sich die Akkommodationsantwort betreffend im Gegensatz zu monokularen Bedingungen bei binokularen Messreihen keine signifikanten Unterschiede zwischen den verschiedenen verwendeten Methoden zur Stimulation und Erfassung der Nahakkommodation ergeben. (83, S.387) Daher eignet sich der binokulare Ansatz besser zum Vergleich eigener Ergebnisse mit anderen Studien, was sicherlich als Vorteil angeführt werden kann. 
Dennoch wurde mit Plättchen in dieser Arbeit eine andere Stimulation der Akkommodation verursacht als mit Spiegel. Bei der Anordnung mit Glasplättchen wurde dem Auge, das gemessen wurde, auch der Akkommodationsreiz geboten, während mit Spiegel ja auf der einen Seite die Zahlen auf der Digitalanzeige eingeblendet wurden, um gleichzeitig kontralateral die Änderungen zu erfassen. Daher war es interessant zu überprüfen, ob sich tatsächlich signifikante Unterschiede zwischen den beiden Methoden nachvollziehen ließen. Dabei ergaben sich sehr hohe signifikante Korrelationen zwischen Plättchen und Spiegel für Messungen der AL bei Nah- und Fernakkommodation $(\mathrm{r}=1,0)$, während die Korrelation für die errechnete Differenz der AL (nahakkommodiertes - unakkommodiertes Auge) etwas niedriger $(\mathrm{r}=0,39)$, aber dennoch signifikant war. Die Mittelwerte der VKT bei Nah- und Fernakkommodation konnten dem Intermethodenvergleich ebenfalls sehr gut standhalten. Dabei wurden Korrelationen von $\mathrm{r}=0,99$ bzw. $\mathrm{r}=1,0$ errechnet. Daraus kann man also schließen, dass sich ipsi- und kontralaterale Stimulation der Akkommodationsantwort bei dieser Versuchsanordnung gleich auf die Änderung der Augenparameter auswirken.

\subsection{Statische Messungen: Vorderkammertiefe und Augenlänge}

\subsubsection{Messungen der Vorderkammertiefe}

Statische Messungen der VKT wurden bereits in zahlreichen longitudinalen und epidemiologischen Studien erforscht, wobei nur selten Kinder in ihnen vorkamen. Der MW der VKT, der in dieser Studie bei emmetropen, im Durchschnitt elfjährigen Kindern gemessen wurde, lag bei 3,62 \pm 0,19 mm. Dieser Wert steht im Einklang mit der Literatur. (73, S.2748) Die Beobachtung anderer Studien, dass die VKT im Alter zwischen 7 und 15 Jahren steigt (25, S.57), konnte auch hier nachvollzogen werden (s. Abbildung 34).

Die größten VKT findet man im Alter zwischen 15 und 20 Jahren. (5, S.1845) Auch in dieser Arbeit wurde die tiefste Vorderkammer in der Gruppe der Jugendlichen beobachtet. Der MW der VKT bei den im Durchschnitt 22-jährigen Testpersonen betrug 3,76 \pm 0,23 mm, was den Ergebnissen anderer Studien entspricht. (5, S. 1845) Auch die Standardabweichung wurde in dieser Altersgruppe in anderen Studien in dieser Größenordnung angegeben. (25, S.56) 
Bei den älteren Probanden wurden im Vergleich zu Kindern und Jugendlichen kürzere Vorderkammern gemessen. Bei den im Mittel 56- jährigen Erwachsenen wurden VKT im Bereich von 3,21 $\pm 0,33 \mathrm{~mm}(\mathrm{MW} \pm \mathrm{SD})$ beobachtet. Eine Verflachung der VKT bei Erwachsenen gegenüber jener bei Jugendlichen war zu erwarten. Sie ist durch Zunahme der Linsenmasse im Rahmen des Alterungsprozesses und die damit einhergehende Vorverlagerung des Linsenzentrums zu erklären. Die Lage der hinteren Linsenfläche ändert sich in Bezug auf Kornea und Retina dabei jedoch nicht. (48, S.1685)

In der Gruppe der Pseudophaken wurden VKT im Bereich von 3,90 \pm 0,20 mm $(\mathrm{MW} \pm \mathrm{SD})$ gemessen. Diese sind offensichtlich deutlich länger als jene, die bei emmetropen Erwachsenen etwa vergleichbaren Alters beobachtet wurden. Aus einigen Studien ist bereits bekannt, dass im Gegensatz zur Messung der AL die Ergebnisse der Untersuchungen der VKT mit Ultraschall und IOL-Master kaum korrelieren. (71, S.85)

Der IOL-Master gibt deutlich größere VKT an als sie mit Ultraschall ermittelt werden. (93, S.27) Der Unterschied der beiden Methoden liegt in der Gruppe der Pseudophaken im Bereich von 0,33 $\pm 0,61 \mathrm{~mm}$ bei nicht-dilatierten Pupillen, wie sie in dieser Arbeit vorliegen. (52, S.92) Trotzdem wird der IOL-Master im klinischen Alltag zur Erfassung der VKT auch bei Patienten nach Kataraktoperationen eingesetzt. Schwierigkeiten bei den Messungen ergeben sich erfahrungsgemäß lediglich bei zu kleinen Pupillen oder bestimmten Haptiken der IOLs.

Dennoch ist es durchaus berechtigt, die Genauigkeit dieser Angaben in Frage zu stellen. Es ist nicht auszuschließen, dass der IOL-Master in manchen Fällen von der Linsenrückfläche statt der -vorderfläche als Bezugspunkt für die Messungen ausgeht. Würde er sich an der Iris ausrichten, ergäben sich falsch zu kurze Werte, was hier jedoch nicht der Fall zu sein scheint. Eventuell behindern auch die Oberflächen- bzw. Brechungseigenschaften der IOLs die korrekte Erfassung der VKT. (52, S.93)

Nichtsdestotrotz stellte sich heraus, dass die individuellen Einzelmessungen der VKT bei den pseudophaken Probanden konstant blieben. Die Standardabweichungen bei den Einzelmessungen waren vereinzelt zwar deutlich höher als die in anderen Gruppen, dennoch lagen sie im Mittel lediglich bei 0,03 mm. Dies ist insofern bei dieser Arbeit von Bedeutung, weil es darum ging, Veränderungen während der Akkommodation zu ermitteln. Gerade bei dieser Gruppe fällt weniger der Absolutwert als eine eventuelle Differenz bei Akkommodation ins Gewicht. Falls also tatsächlich eine Orientierung des Geräts an der 
Linsenrückfläche erfolgt sein sollte, so fand diese konstant bei jeder Messung des Probanden statt. Daher kann man auch annehmen, dass, falls eine Bewegung der Linse während Nahakkommodation stattfinden würde, diese mit hoher Wahrscheinlichkeit an der Differenz der VKT zwischen unakkommodiertem und akkommodiertem Auge auch bei pseudophaken Probanden abzulesen wäre. Darauf wird allerdings im Weiteren näher eingegangen.

\subsubsection{Messungen der Augenlänge}

In den vergangenen Jahren wurden bereits Studien publiziert, die die partielle KohärenzInterferometrie als eine zuverlässige und gut reproduzierbare Methode für AL-Messungen bei Kindern im Alter von 3 bis 12 Jahren auswiesen. (77, S.985) Diese Erfahrung konnte in dieser Arbeit mit verschiedenen Alterstufen bestätigt werden. Es waren schnelle, präzise und konstante Messungen der AL in der Kindergruppe möglich. Die intraindividuellen Einzelwerte streuten lediglich um 0,01 bis $0,02 \mathrm{~mm}$.

Während der MW der AL bei den jüngsten Probanden 23,19 \pm 0,49 mm betrug, waren in der Gruppe der Jugendlichen signifikant größere Achslängen zu messen: 23,94 \pm 0,79 mm (MW \pm SD). Der Mittelwert, sowie die Standardabweichung entsprechen den AL, die in anderen Studien bei Emmetropen dieser Altersstufe beobachtet wurden. (21, S.2144) In der Literatur wurde bereits öfter diskutiert, dass, wie hier im Vergleich der Gruppen der Kinder und Jugendlichen auffällt, größere Achslängen auch mit tieferen Vorderkammern des Auges einhergehen. (40, S.336)

Erwachsene (AL: 23,66 $\pm 0,73 \mathrm{~mm}(\mathrm{MW} \pm \mathrm{SD}))$ und Jugendliche unterscheiden sich in Bezug auf AL nicht signifikant. Eine Änderung der AL im Zusammenhang mit dem Alterungsprozess wurde weder beobachtet, noch war sie erwartet worden.

Die AL der Pseudophaken unterscheidet sich ebenfalls nicht signifikant von jener der Erwachsenen, auch wenn sie im Mittel etwas kleiner ist $(23,05 \pm 0,79 \mathrm{~mm}(\mathrm{MW} \pm \mathrm{SD}))$. Genauso wie bei den Messungen mit Ultraschall, muss auch beim IOL-Master beachtet werden, dass sich statt der natürlichen Linse eine künstliche mit etwas anderen geometrischen Eigenschaften im Auge befindet. Wenn man dieser Tatsache nicht Rechnung tragen würde, ergäben sich bei der Messung der AL systematische Fehler von etwa 0,12 mm gemittelt für verschiedene Linsentypen. (34, S.589) In dieser Arbeit wurde daher vor der Messung der AL bei Pseudophaken der jeweilige Linsentyp am Gerät eingestellt, so dass vom IOL-Master 
automatisch der Korrekturfaktor mitberücksichtigt werden konnte. Es wurden keine auffälligen Unterschiede, weder bei VKT- noch bei AL-Messungen, zwischen Silikon- und Acryllinsenträgern beobachtet.

\subsection{Augenparameter als Spiegel der Akkommodationsantwort}

Die Vorgänge, die während der Akkommodation im Auge stattfinden, sind seit Jahren im Groben bekannt. Dennoch war präzises Wissen um die Dimensionen der Augenstrukturen und ihrer Änderungen während der Naheinstellung in den letzten Jahren von großem ophthalmologischem Interesse. Der Frage, ob Akkommodation und Naharbeit im Zusammenhang mit Myopie stehen, wurde vielfach nachgegangen. (27, S.208) Dennoch ist die Änderung der AL während der Naheinstellung bislang ein sehr umstrittener Punkt, der in diesem Kapitel, neben dem Verhalten der VKT während der Akkommodation, Beachtung finden soll.

\subsubsection{Vorderkammertiefe}

Zunächst einmal lohnt es sich die Vorgänge während der Akkommodation bei den drei „gesunden“ Probandengruppen genauer zu besprechen.

\subsubsection{Abnahme der Vorderkammertiefe bei Nahakkommodation}

\subsection{Kinder}

Bei Kindern wurden bei der Nahakkommodation Änderungen der VKT bis hin zu 0,18 mm im Vergleich zum unakkommodierten Zustand beobachtet, der MW der gemessenen Differenzen lag allerdings bei $0,11 \pm 0,04 \mathrm{~mm}$. Da bei emmetropen Kindern die Akkommodationsamplitude höher ist als bei emmetropen Jugendlichen, ist die Überlegung nahe liegend, dass sich analog dazu die größere Verflachung der Vorderkammer während der Naheinstellung in der Gruppe der Kinder ergeben müsste. Tatsächlich ist es aber so, dass die Differenzen der VKT bei Jugendlichen im Mittel 0,03 mm länger sind als jene bei Kindern. Naheliegend ist sicherlich die Erklärung, dass bei einem Nahstimulus in $16 \mathrm{~cm}$ Entfernung die Akkommodation bei Kindern noch nicht maximal angeregt wurde.

Bei Betrachtung der Literatur, die zur Akkommodation bei Kindern vorliegt, werden zudem verschiedene andere Faktoren diskutiert. Es fällt zunächst auf, dass kaum Studien mit unter Zehnjährigen durchgeführt wurden. Falls es solche gab, so stellten sie mitunter fest, dass 
Kinder deutlich unterhalb der ihnen von Duane zugesprochenen und bis heute noch anerkannten hohen Akkommodationsamplitude lagen. (97, S.246) Andere schlugen wiederum vor, dass zwischen Kindern im Alter von sechs bis sieben Jahren und jenen, die schon zur Schule gingen, eine grundsätzliche Unterscheidung getroffen werden müsse, wenn man die Akkommodationsfunktion betrachtet. (42, S.97)

Viele Arbeitsgruppen stellten sogar Ergebnisse bei Kindern unter acht Jahren grundsätzlich in Frage und machten die Beobachtung, dass mit steigendem Alter die akkommodativen Fähigkeiten, bzw. die Auffassungsgabe zunehmen. (108, S.178) Bei den in dieser Arbeit durchgeführten Messungen wurde jedoch der Eindruck gewonnen, dass Kinder gut in der Lage sind, Erklärungen und Aufforderungen zuverlässig zu folgen. Kürzeren Konzentrationsspannen bei Kindern wurde durch eine geringere Anzahl der Messungen im Vergleich zu anderen Gruppen Rechnung getragen. Der Vorteil dieser Arbeit ist, dass sie im Gegensatz zu vielen anderen Studien bei Kindern (97, S.248; 108, S.178) ohne deren subjektive Angaben auskommen konnte. Während sie die ihnen gezeigte Zahl ablasen und somit ihr Auge auf Nähe oder Ferne einstellten, konnten objektive Messungen vorgenommen werden.

\subsection{Jugendliche}

Im Gegensatz zu Kindern gibt es in der Studienlandschaft gute Vergleichsmöglichkeiten für Ergebnisse in der Gruppe der Jugendlichen. Bei ihnen verflachte sich die Vorderkammer im Durchschnitt um 0,14 mm \pm 0,03 mm $(\mathrm{MW} \pm \mathrm{SD})$, jedoch wurden Differenzen bis hin zu 0,20 $\mathrm{mm}$ beobachtet. Diese Werte stehen im Einklang mit denen anderer Arbeitsgruppen. Von Bayramlar et al. wurden Verflachungen der VKT bei Jugendlichen in der Größenordnung von 0,12 mm gemessen. (6, S.306) Drexler et al. ermittelten in einer Gruppe junger Erwachsener Differenzen von 0,05 bis $0,30 \mathrm{~mm}$, im Durchschnitt also eine Verringerung der VKT bei Nahakkommodation um 0,18 mm. (19, S.2795)

\subsection{Erwachsene}

Deutlich kleinere Änderungen der Vorderkammer wurden in der Gruppe der Erwachsenen gemessen. Diese beliefen sich im Durchschnitt auf 0,07 $\pm 0,02 \mathrm{~mm}$ (MW $\pm \mathrm{SD}$ ). Ein zunehmendes Nachlassen der akkommodativen Fähigkeit konnte an den Werten der VKTDifferenz hervorragend nachvollzogen werden (siehe Abbildung 36). Beim Berechnen der Regressionsgerade ergab sich ein linearer Abfall der Akkommodationsantwort der Vorderkammer mit dem Alterungsprozess.

Interessant ist, dass das Alter, in dem Presbyopie auftritt, variiert. Unterschiede bedingt durch Rasse oder geographische Breiten werden diskutiert. (41, S.171; 105, S.515) Tatsächlich 
kommt es bei Menschen, die näher am Äquator wohnen, auch früher zur Altersweitsichtigkeit. Während in Norwegen die Presbyopie mit durchschnittlich 46 Jahren anfängt, beginnt sie in Indien bereits mit 37. (65, S.191) Wann genau die Presbyopie bei den in dieser Arbeit vorgestellten Erwachsenen aufgetreten ist, bzw. auftreten wird, kann nicht ermittelt werden, jedoch wurde eines unmissverständlich deutlich: auch innerhalb der Erwachsenengruppe gab es starke Unterschiede zwischen den jüngeren und den älteren Probanden. Die maximale Verflachung der VKT von 0,11 mm wurde bei einer 48-jährigen Person gemessen. Die Minima von 0,04 mm jedoch ergaben sich bei den Ältesten in der Gruppe, 66 und 69 Jahre alt. Diese Beobachtungen decken sich mit jenen anderer Studien, die um das Alter von 55 Jahren deutlich reduzierte akkommodative Fähigkeiten ermitteln. $(74,1435)$ Die Werte, die bei dem älteren Teil der Erwachsenen gemessen wurden, entsprechen Ergebnissen anderer Arbeitsgruppen, die signifikante Differenzen der VKT bei Nahakkommodation um $0,04 \pm$ 0,02 $\mathrm{mm}$ in der Gruppe der Altersweitsichtigen feststellen konnten. (47, S.797) Entscheidend hierbei ist, dass in dieser Arbeit die Akkommodation auch bei älteren Erwachsenen mit physiologischen Mitteln hervorgerufen wurde, zumal inzwischen bekannt ist, dass das vielfach verwendete Pilocarpin in der Gruppe der Presbyopen als Superstimulus wirkt und die gemessenen Differenzen fälschlicherweise zu hoch ausfallen. (47, S.795)

\subsubsection{2 Änderung der Vorderkammertiefe bei Fernakkommodation}

Was die Fernakkommodation betrifft, wurden bei Probanden aller Gruppen meistens keine Änderungen im Vergleich zum unstimulierten Zustand gemessen. Zum Teil wurden geringe Zunahmen der VKT im Bereich von 0,01 mm beobachtet, aber bei einigen wenigen auch Verflachungen der Vorderkammer in dieser Größenordnung. Schwankungen der VKT um $0,01 \mathrm{~mm}$ bei der Ferneinstellung werden von anderen Arbeitsgruppen ebenfalls diskutiert. $(19$, S.2797)

\subsubsection{Augenlänge}

Wieso die AL als Augendimension von großer Bedeutung ist, wird bei Betrachtung der Theorie zum Vorgang der „Emmetropisation“ deutlich. Mit dem Wachstum des Kindes machen Hornhaut, Linse und AL koordinierte Veränderungen durch. Während die Hornhaut und die Linse an Brechkraft verlieren, nimmt die AL zu, so dass auf der Netzhaut ein scharfes Bild entstehen kann. Da die Linse bis zum Alter von sechs Jahren 20 dpt an Brechkraft verliert, muss zum Ausgleich die AL um etwa 5-6 mm zunehmen. Dabei bedeutet die 
Vergrößerung der AL um 1 mm eine Zunahme der Brechkraft des Auges um etwa 3 dpt. (65, S.163)

Da die Inzidenz der Myopie mit Erreichen des Alters von zehn Jahren deutlich ansteigt, muss man sich die Frage stellen, ob dies mit einer Fehlsteuerung des oben beschriebenen Mechanismus des Abfalls der Linsenbrechkraft bei gleichzeitiger Augenverlängerung zusammenhängen könnte. Es wurde bereits vielfach diskutiert, dass vermehrte Naharbeit (wie z.B. Lesen) auch verstärkt mit Myopie einhergeht. (76, S.2794; 88, S.332; 92, S.233; 82, S.159)

Daher wird häufig die Hypothese aufgestellt, dass die lang andauernde Akkommodation die Verbindung zwischen der Lederhaut und dem Ziliarkörper schwächt und damit gleichzeitig auch die einschränkende Wirkung, die diese Verbindung auf das Augenwachstum ausübt, mindert. Das würde mit einer Zunahme der AL bei myopen Menschen einhergehen, was in zahlreichen Studien auch beobachtet wird. (65, S.164)

Andere stellen wiederum die Behauptung auf, dass der Akkommodationsvorgang zu einer direkten Veränderung der AL führt. (94, S.76) Dieser Hypothese wurde in dieser Arbeit nachgegangen.

\subsubsection{1 Änderung der Augenlänge bei Nahakkommodation}

In allen vier Gruppen wurden ähnliche Ergebnisse bei der Betrachtung der AL während Nahakkommodation erhoben. In etwa 30 bis $47 \%$ der Fälle ( $N=5$ bis 7) konnte gar keine Änderung der AL beim Blick in die Nähe ermittelt werden. Bei den restlichen Probanden wurden signifikante Verlängerungen der AL in der Größenordnung von $10 \mu \mathrm{m}$ beobachtet. Dieser Wert deckt sich mit den Resultaten von Drexler et al., die in einer Studie mit jungen Erwachsenen eine Zunahme der AL von 12,7 $\mu \mathrm{m}$ gemessen haben. (21, S.2140) Vereinzelt (bei 3 von 60 Probanden) wurden in dieser Arbeit auch größere Änderungen von $20 \mu \mathrm{m}$ oder bei einem jugendlichen Probanden sogar $40 \mu \mathrm{m}$ bei Nahakkommodation gemessen. Werte von Mallen et al., die im Durchschnitt eine Verlängerung der AL um MW 40 m (62, S.1251) oder jene von Shum et al., die sogar Änderungen um MW $50 \mu \mathrm{m}$ (94, S.76) beobachteten, konnten in dieser Arbeit jedoch nicht bestätigt werden. Jene von Shum et al. wurden allerdings in der Fachpresse bereits in Frage gestellt. (107, S.682)

Dennoch gilt es zu überlegen, wie man diese beobachteten kleinen Änderungen im $\mu \mathrm{m}$ Bereich erklären könnte. Einige Arbeitsgruppen schlagen vor, dass die mit der Akkommodation einhergehende Kontraktion des Ziliarmuskels zu einer Abnahme des skleroidalen Umfangs führt, indem sie an der Choroidea einen nach vorne innen gerichteten 
Zug ausübt. Dass eine Verkleinerung des skeroidalen Umfangs mit einer Verlängerung der Axiallänge des Auges einhergeht, ist ein nachvollziehbarer Denkansatz. (21, S.2140) Auf der anderen Seite muss man aber auch sehen, dass die AL selbst im Tagesverlauf größeren Schwankungen unterliegt als sie in dieser Arbeit während der Akkommodation gemessen werden konnten. (98, S.63) Van der Heijde et al. stellten gar Fluktuationen der AL im Bereich von 2 bis $10 \mu \mathrm{m}$ im Zusammenhang mit Variationen der Herzfrequenz und der Atmung fest. (102, S.216)

Darüber hinaus könnte die Messmethode des IOL-Masters selbst eine Erklärung für die gemessene Vergrößerung der AL bei Akkommodation liefern. Tatsächlich misst der IOLMaster nämlich die optische Axiallänge des Auges. Hieraus wird die Augenlänge durch Division durch den mittleren Brechungsindex des Auges errechnet und ausgegeben. Die bei der Akkommodation auftretende Verdickung der Linse erhöht allerdings den mittleren Brechungsindex des Auges. Damit kann selbst bei gleich gebliebener tatsächlicher Augenlänge durch die bei der Akkommodation auftretende Erhöhung des mittleren Brechungsindex eine Vergrößerung der optischen Augenlänge gemessen werden. Nach Atchison et al. kann dieser systematische Fehler je nach Stärke der Akkommodation bei bis zu $18 \mu \mathrm{m}$ liegen. (2, S.286)

Bei Kunstlinsenträgern kann jedoch davon ausgegangen werden, dass es während der Naheinstellung nicht zu einer Linsenverdickung und damit auch nicht zu einer Änderung des mittleren Brechungsindex kommt. Folglich kann in der vorliegenden Studie obige Fehlerquelle zumindest in der Gruppe der Pseudophaken, bei der aber auch eine Verlängerung der AL um $10 \mu \mathrm{m}$ bei Nahakkommodation gemessen wurde, mit Gewissheit ausgeschlossen werden.

Dennoch müssen die Änderungen der AL in der Größenordnung von $10 \mu \mathrm{m}$ insgesamt in Frage gestellt werden, da die Messgenauigkeit des IOL-Masters genau bei diesen $10 \mu \mathrm{m}$ liegt, sowie minimale Schwankungen der Messachse, die es bei kleinsten Augenrotationen bereits geben könnte (46, S.65), nicht vollständig ausgeschlossen werden können.

\subsubsection{Rolle der Konvergenz bei Zunahmen der Augenlänge}

Das Argument von Bayramlar et al., dass eventuell beobachtete Zunahmen der AL auch von der Konvergenz verursacht sein könnten, muss in dieser Arbeit besondere Beachtung finden. (6, S.304) Da das Auge, das gerade nicht vermessen wurde, nicht abgedeckt war, was natürlich mit kontralateraler Stimulation mittels Spiegel erst gar nicht umsetzbar gewesen 
wäre, ist es denkbar, dass die gemessenen Veränderungen der Augenparameter ein Effekt von beidem, Akkommodation und Konvergenz, sind.

Inwiefern mechanische oder physiologische Faktoren tatsächlich als Erklärung für die gemessenen Mikrofluktuationen dienen könnten, wäre dennoch weiter zu eruieren. Als sicher gilt aus meiner Sicht, dass diese gemessene AL-Veränderung, die mit einem Gewinn von maximal 0,03 dpt einhergeht, nicht entscheidend zur Zunahme der Brechkraft bei der Naheinstellung beiträgt.

\subsection{Kunstlinsen und Akkommodation}

Da sich die Pseudophaken in ihrer Änderung der VKT bei Nahakkommodation deutlich von den anderen Gruppen unterschieden haben, werden sie an dieser Stelle getrennt diskutiert.

\subsection{1 „Pseudoakkommodation“}

In der Literatur ist bereits bestens bekannt, dass pseudophake Patienten unabhängig von der Kontraktion des Ziliarmuskels Mechanismen vorweisen, die zu einer Anhebung des fernkorrigierten Nahvisus führen können. Man spricht hierbei von „Pseudoakkommodation“. Als Ursachen werden hierbei veränderte statische Eigenschaften des Auges z.B. durch eine Zunahme der Augenlänge, durch Puppillenverengung und die damit einhergehende Vergrößerung der Tiefenschärfe oder die Multifokalität von Hornhaut und Linse diskutiert. (54, S.822; 26, S.1178; 60, S.397)

\subsection{2 Änderung der Vorderkammertiefe bei Pseudophaken während der Nahakkommodation}

Die Frage ob bei Pseudophaken eine echte Akkommodation, die durch die Interaktion zwischen dem sich kontrahierenden Ziliarmuskel einerseits und den Zonulafasern, Linsenkapsel und Kunstlinse andererseits, zustande kommt, statt findet, ist jedoch heftig umstritten.

An dieser Arbeit nehmen 15 Probanden mit Silikon- oder Acryllinsen teil. Bei Nahakkommodation konnten bei der Mehrheit von 12 (Plättchen-), bzw. 9 (Spiegelmethode) pseudophaken Probanden gar keine Änderungen der VKT gemessen werden. Diese 
Beobachtung deckt sich mit jener der Arbeitsgruppe Kriechbaum et al., die ebenfalls keine Änderung der VKT bei Stimulation der Nahakkommodation mit einem physiologischen Reiz ermitteln konnten. (53, S.453) Es gibt jedoch auch Publikationen, die bei jungen Patienten und ähnlicher Versuchsanordnung Verflachungen der VKT in der Größenordnung von 0,42 mm angeben. (59, S.562) Diese müssen nach den aus dieser Arbeit gewonnenen Erkenntnissen stark in Frage gestellt werden, zumal einerseits die größte gemessene Differenz bei Pseudophaken in dieser Arbeit $0,01 \mathrm{~mm}$ betrug und andererseits selbst die in der Gruppe emmetroper Jugendlicher beobachteten Differenzen der VKT deutlich unter 0,42 mm lagen.

Wieso in der Gruppe der Pseudophaken also keine Änderung der VKT gemessen wurde, könnte verschiedene Gründe haben. Der akkommodative Stimulus war in den anderen Gruppen ausreichend groß um eine Verflachung der VKT hervorzurufen, also muss die Ursache an den pseudophaken Probanden selbst liegen. Möglich wäre, dass der im Alter zwar noch funktionsfähige Ziliarmuskel einfach zu schwach ist um die IOL zu bewegen. (53, S.457)

Denkbar wäre auch, dass der Muskel sich zwar gut kontrahiert, die Bewegung aber insuffizient auf die IOL übertragen wird. (57, S.455)

Inzwischen hat man berechnet, dass mit einer Vorwärtsbewegung der IOL um 1,0 mm eine Zunahme der Brechkraft um 0,8 bis 2,3 dpt, je nach Augenlänge und Radius der Kornea, einhergeht. (70, S.2069). Die in dieser Arbeit gemessenen Linsen sind jedoch von einer 1,0 mm großen Abnahme der VKT weit entfernt.

\subsubsection{Ausblick}

Da die Presbyopie bislang eine unvermeidbare Folge des Alterungsprozesses darstellt, ist das Interesse groß, eine Lösung für das schwindende Akkommodationsvermögen zu finden.

\subsubsection{Linsenmodelle}

Die Suche nach dem optimalen Ersatz für die natürliche Linse hat schon vor langer Zeit begonnen. Inzwischen gibt es bifokale, multifokale oder diffraktive Linsen. Dennoch erlauben diese im besten Falle eine Verbesserung des unkorrigierten Nahvisus auf Kosten der Kontrastempfindlichkeit und des Dämmerungsehens. (54, S.820)

Als weiterer Lösungsansatz wurde 2000 die Hinterkammerlinse 1CU (Human Optics, Erlangen) vorgestellt. Diese stützt sich auf die Beobachtungen, dass der Ziliarmuskel auch im Alter einen Teil seiner Kontraktilität behält. (99, S.1162) Die aus faltbarem hydrophilem 
Acrylat bestehende Linse soll Akkommodation vermitteln, indem durch die Kontraktion des Ziliarmuskels eine Konfigurationsänderung ihrer Haptiken hervorgerufen wird, die letztlich zu einer Vorwärtsbewegung der Linsenoptik („Focus-Shift-Prinzip“) führt. (54, S.821) Die Ergebnisse der klinischen Studien in Bezug auf tatsächliche Änderungen der VKT gehen bei der 1CU-Linse jedoch deutlich auseinander. Während Küchle et al. eine Differenz der VKT von durchschnittlich 0,63 mm mit medikamentöser Stimulation gemessen haben (54, S.823), konnten Kriechbaum et al. eine Vorwärtsbewegung von lediglich $0,01 \mathrm{~mm}$ mit physiologischer bzw. 0,20 mm unter Pilocarpin-Einfluss nachweisen. (53, S.457) Alle Ergebnisse, die jedoch unter Pilocarpin-Zuhilfenahme erarbeitet wurden, sind aber, wie bereits erwähnt, mit Vorsicht zu interpretieren, da so falsch zu hohe VKT-Änderungen gemessen werden. (3, S.809) Andere Autoren wiederum, wie z.B. Schneider et al. konnten keine signifikanten Unterschiede zwischen der 1CU-Linse und PMMA-Linsen mit rigiden Haptiken ermitteln. (90, S.2005) Kontrovers wird auch das Auftreten einer Kapselfibrose nach 1CU-Implantation, die zu einer Dislokation der Linse führen könnte, diskutiert. (54, S.823; 63, S.1307)

Nach ähnlichem Prinzip wie die 1CU-Linse funktionieren auch die von Cumming oder Payer vorgestellten AT-45- (17, S.2005) bzw. BioComFold-Hinterkammerlinsen. Inwiefern diese vorteilhafter gegenüber gewöhnlichen IOLs sind, ist jedoch noch unklar. (54, S.823)

Ein weiterer interessanter Ansatz wurde in der Theorie vorgestellt: Linsen mit „Dual-Optik“Design. Bei diesen ist eine starke anteriore Sammeloptik über abgefederte Haptiken mit einer posterioren statischen Zerstreuungsoptik verbunden. (64, S.1083) In mathematischen Studien erwies sich dieses Modell als vorteilhaft gegenüber den bisher beschriebenen „Mono-Optik“Linsen, da es mit dem gleichen Betrag an linearer Verschiebung der anterioren Optik entlang der Augenachse größere Änderungen der Brechkraft hervorrufen würde, als es z.B. die 1CUHinterkammerlinse tun könnte. (57, S.451)

\subsubsection{Alternativen zu Kunstlinsen: „Phako-Ersatz“ und skleraexpandierende Operationsverfahren}

Neben verschiedenen Linsenmodellen gibt es auch völlig andere Vorschläge zur Lösung des Problems der Presbyopie. Da bekannt ist, dass mit dem Alter die Linse zwar härter wird, aber die Kapsel ein gewisses Maß an Elastizität beibehält, werden Überlegungen laut, die Linse durch künstliches biokompatibles Material zu ersetzen, das ihre in der Jugend vorhandenen 
Eigenschaften nachahmt. Diesem Prinzip der Linsenkapselwiederauffüllung (,lens-refilling“) wurde von verschiedenen Arbeitsgruppen nachgegangen.

Eine Methode wurde bereits 1986 von Parel et al. unter dem Namen „Phako-Ersatz“ vorgestellt. (75, S.165) Vor allem im Bereich der kindlichen Katarakt oder bei hyperopen Augen könnte diese Technik, die eine Kapsulorhexis von 1,5 mm Durchmesser und Linsenersatz durch vorbehandeltes polymeres Silikon beinhaltet, in Zukunft zum Einsatz kommen. (100, S.744; 38, S.405) Erste Operationen an Primatenaugen konnten viel versprechende Ergebnisse vorweisen. (32, S.550; 33, S.471) Dennoch ist dieser originelle Ansatz bisher nur experimenteller Natur. (54, S.821)

Von Nishi et al. wurde der Einsatz eines aufblasbaren Silikonballon mit einem Stöpsel zum Verschluss der Minikapsulorhexis bei Cyanomolgusaffen erfolgreich durchgeführt. (72, S.1358)

Assia et al. wiederum entwickelten eine expansible Hydrogellinse, die im dehydrierten Zustand implantiert wird, um anschließend durch Aufnahme von Kammerwasser zur geplanten Größe zu wachsen. (3, S.810)

Obwohl diese Überlegungen allesamt auf großes Interesse stoßen, konnten sie sich in den letzten Jahren kaum weiterentwickeln und für den Einsatz an Menschen empfehlen. Als problematisch wird vor allem die genaue Vorausberechnung der abschließenden Brechkraft diskutiert. Bemängelt wird überdies, dass eine homogene Auffüllung des Kapselsacks ohnehin nicht die optischen Vorteile der natürlichen Linse mit ihren abgestuften Brechungsindizes liefern kann. (13, S.3)

Neben den „lens-refilling“- gibt es auch skleraexpandierende Operationsverfahren. Diese sind durch die von Schachar aufgestellte, in der Einleitung bereits besprochene, Theorie zur Akkommodation inspiriert. Sie stützen sich vor allen Dingen auf die Beobachtung, dass mit zunehmendem Alter der Abstand zwischen dem Ziliarring und dem Linsenäquator abnimmt. Dadurch sieht man die Funktion der Zonulafasern, ihre Spannung und somit die Krümmung der Linse zu ändern, als eingeschränkt. Man wendet daher Verfahren an, die die Spannung der Zonulafasern erhöhen sollen, in der Annahme, dass diese anschließend die gealterte Linse verformen und damit die Akkommodationsamplitude erhöhen können. Die Wirksamkeit der skleraexpandierenden Verfahren ist jedoch bislang fraglich und das Risiko verschiedener post-operativer Komplikationen scheint signifikant hoch zu sein. (13, S.2) 
Abschließend gilt es zu bemerken, dass die oben dargestellten Lösungsansätze nur wenige von einer Unmenge an Bemühungen, die Akkommodation wiederherzustellen, sind. Bislang sind noch viele Fragen offen, aber die optimale Lösung scheint zum Greifen nahe. Die Entwicklung einer zuverlässig „akkommodierenden Kunstlinse“ könnte das gesamte Denken in der Ophthalmologie revolutionieren. Angesichts des stetig steigenden Anteils der älteren Bevölkerung, könnte eine solche Kunstlinse für viele nicht nur die Lösung für Katarakt sondern auch für Presbyopie bedeuten. 


\section{Zusammenfassung}

HINTERGRUND: Mit dem Bestreben, Akkommodation auch im Alter möglich zu machen, wird es immer wichtiger, die dabei stattfindenden Vorgänge im Auge in allen Einzelheiten zu verstehen. In dieser Arbeit wurden Längenverhältnisse in der Sehachse während der Akkommodation bei unterschiedlichen Altersgruppen und Linsenstatus untersucht.

METHODEN: Bei jeweils 15 emmetropen Probanden im Alter von 10,5 $\pm 1,9,21,9 \pm 2,1$ und 55,7 \pm 7,3 Jahren (MW \pm SD), sowie bei 15 Pseudophaken (59,9 \pm 7,8 Jahre) wurden die Parameter Vorderkammertiefe (VKT) und Augenlänge (AL), sowie ihre Änderung bei Akkommodation ermittelt. Die Messungen erfolgten mittels IOL-Master und einer eigens angefertigten Zusatzeinrichtung zur physiologischen Auslösung der Akkommodation einerseits am ipsilateralen, anderseits am kontralateralen Auge. Beide Methoden wurden im Vergleich zwischen zwei Untersuchern auf Reproduzierbarkeit überprüft.

ERGEBNISSE: Die Messergebnisse der angewendeten Methoden ( $r>0,90)$, sowie jene zwischen zwei Untersuchern $(r>0,98)$ korrelierten signifikant $(p=0,00 ;$ bzw. $p<0,004)$ miteinander. Es wurde beobachtet, dass die VKT von Kindern $(3,62 \pm 0,19 \mathrm{~mm})$ kleiner ist als jene bei Jugendlichen $(3,76 \pm 0,23 \mathrm{~mm})$. Mit steigendem Alter wurde jedoch eine Abnahme der VKT beobachtet (Erwachsene: 3,21 $\pm 0,33 \mathrm{~mm}$ ). Bei Nahakkommodation wurden die größten Verkürzungen der VKT bei Jugendlichen $(0,14 \pm 0,03 \mathrm{~mm})$, gefolgt von Kindern $(0,11 \pm 0,03 \mathrm{~mm})$ gemessen. Während innerhalb der Erwachsenengruppe Abnahmen der VKT um 0,07 $\pm 0,02 \mathrm{~mm}$ beobachtet wurden, konnten bei Pseudophaken vergleichbarer Altersgruppe in der Mehrzahl der Fälle gar keine Änderungen der VKT gemessen werden. Auffällig war auch, dass sich innerhalb der Erwachsenengruppe deutliche altersbedingte Unterschiede ergaben. So wurden die kleinsten Verflachungen der VKT bei Nahakkommodation $(0,04 \mathrm{~mm}$ ) bei den ältesten (66 und 69 Jahre alt) und die größten Differenzen der VKT $(0,11 \mathrm{~mm})$ bei den jüngsten erwachsenen Probanden (48 Jahre alt) ermittelt. Die AL nahm bei allen Gruppen während Nahakkommodation signifikant $(p<0,02)$ um $0,01 \pm 0,01 \mathrm{~mm} \mathrm{zu}$.

DISKUSSION: Mittels physiologischer Stimulation ist bei Pseudophaken kaum eine Änderung der VKT bei Nahakkommodation messbar. Die beobachtete Verlängerung der AL 
um $10 \mu \mathrm{m}$ ist im Zusammenhang mit der Präzision des IOL-Masters und atmungs- sowie herzfrequenzabhängigen Schwankungen der AL kritisch zu bewerten. 


\section{Schlussfolgerungen}

Mit präziser klinisch-experimenteller Erforschung des Themas Akkommodation war es das Bestreben dieser Arbeit, einen objektiven und wissenschaftlich fundierten Beitrag zu den Bemühungen, Akkommodation zu verstehen und wiederherzustellen, zu leisten.

Folgende Schlussfolgerungen können aus den gemachten Beobachtungen gezogen werden:

- Die in dieser Arbeit vorgestellte Zusatzeinrichtung eignet sich zur physiologischen Stimulation der Akkommodation. Die beiden verwendeten Methoden (Plättchen/Spiegel) sind sehr gut reproduzierbare und untersucherunabhängige Mittel zur ipsi- bzw. kontralateralen Auslösung der Akkommodation.

- Ein signifikanter Unterschied zwischen ipsi- und kontralateraler Akkommodationsantwort konnte nicht ausgemacht werden.

- Die Vorderkammer nimmt von Kinderjahren bis in die Jugend an Tiefe zu, um dann mit steigendem Alter deutlich abzunehmen.

- Die größten Verkürzungen der VKT ergaben sich bei der Nahakkommodation in den Gruppen der Jugendlichen und der Kinder. Deutlich kleinere Differenzen der VKT wurden in der Gruppe der Erwachsenen beobachtet. Innerhalb der Erwachsenengruppe konnten anhand der mit zunehmendem Alter nachlassenden Verkürzung der VKT bei Nahakkommodation die Folgen der Presbyopie nachvollzogen werden.

- Bei Pseudophaken findet keine echte Akkommodation, die zu einer signifikanten Zunahme der Brechkraft führen könnte, statt. In der deutlichen Mehrzahl der beobachteten Kunstlinsenträger konnte gar keine Änderung der VKT bei Nahakkommodation gemessen werden.

- Die AL nimmt bei Nahakkommodation signifikant um 0,01 $\mathrm{mm}$ zu. Inwiefern diese Verlängerung im Mikrometerbereich tatsächlich von Vorgängen während der Akkommodation herrührt, oder eine Folge von messbedingten oder atmungs- und herzfrequenzabhängigen Schwankungen darstellt, wäre weiter $\mathrm{zu}$ eruieren. 


\section{Literaturverzeichnis}

1. Abraham L.M., Kuriakose T., Sivanandam V., Venkatesan N., Thomas R., Muliyil J., Amplitude of accommodation and its relation to refractive errors, Indian $J$ Ophthalmol. 53 (2005) 105-108

2. Atchison D.A., Smith G., Possible errors in determining axial length changes during accommodation with the IOLMaster, Optom Vis Sci. 81 (2004) 283-286

3. Auffarth G.U., Akkommodative Intraokularlinsen. Kann eine Kunstlinse akkommodieren?, Ophthalmologe 11 (2002) 809-810

4. Augustin A.J., Differentialdiagnose. In „Augenheilkunde", Springer, Berlin Heidelberg, 2001, 2. Auflage, 823-883

5. Baikoff G., Lutun E., Ferraz C., Wei J., Static and dynamic analysis of the anterior segment with optical coherence tomography. J Cataract Refract Surg. 30 (2004) 18431850

6. Bayramlar H., Çekiç O., Hepşen I.F., Does convergence, not accommodation, cause axial length elongation at near? Ophthalmic Res. 31 (1999) 304-308

7. Beauchamp R., Mitchell B., Ultrasound measures of vitreous chamber depth during ocular accommodation. Am J Optom Physiol Opt. 62 (1985) 523-532

8. Bedienungsanleitung IOLMaster, Softwareversion 3.xx, Firma Carl Zeiss Meditec, 2004, 1-90

9. Beebe D.C., The Lens. In „Adler`s physiology of the eye", Kaufman P.L., Alm A. (Hrsg.), Mosby, Inc., St. Louis, 2003, 10.Auflage, 117-158

10. Beers A.P., van der Heijde G.L., Age- related changes in the accommodation mechanism. Optom Vis Sci. 73 (1996) 235-242 
11. Carkeet A., Saw S.M., Gazzard G., Tang W., Tan D.T., Repeatability of IOLMaster biometry in children. Optom Vis Sci. 81 (2004) 829-834

12. Charman W.N., Heron G., Fluctuations in accommodation: a review. Ophthalmic Physiol Opt. 8 (1988) 153-164

13. Charman W.N., Restoring accommodation: a dream or an approaching reality? Ophthal Physiol Opt. 25 (2005) 1-6

14. Coleman D.J., Fish S.K., Presbyopia, accommodation, and the mature catenary. Opthalmology. 108 (2001) 1544-1551

15. Coleman D.J., Unified model for accommodative mechanism. Am J Ophthalmol. 69 (1970) 1063-1079

16. Connors R. 3rd, Boseman P. 3rd, Olson R.J., Accuracy and reproducibility of biometry using partial coherence interferometry. J Cataract Refract Surg. 28 (2002) 235-238

17. Cumming J.S., Slade S.G., Chayet A., AT-45 Study Group, Clinical evaluation of the model AT-45 silicone accommodating intraocular lens: results of feasibility and the initial phase of a Food and Drug Administration clinical trial. Ophthalmology. 108 (2001) 2005-2010

18. Dick G.L., Relationship between axial length and chromatic refraction of the eye. Ophthal Physiol Opt. 12 (1992) 443-447

19. Drexler W., Baumgartner A., Findl O., Hitzenberger C.K., Fercher A.F., Biometric investigation of changes in the anterior eye segment during accommodation. Vision Res. 37 (1997) 2789-2800 
20. Drexler W., Baumgartner A., Findl O., Hitzenberger C.K., Sattmann H., Fercher A.F., Submicrometer precision biometry of the anterior segment of the human eye. Invest Ophthalmol Vis Sci. 38 (1997) 1304-1313

21. Drexler W., Findl O., Schmetterer L., Hitzenberger C.K., Fercher A.F., Eye elongation during accommodation in humans: differences between emmetropes and myopes. Invest Ophthalmol Vis Sci. 39 (1998) 2140-2147

22. Dubbelman M., Van der Heijde G.L., Weeber H.A., Change in shape of the aging human crystalline lens with accommodation. Vision Res. 45 (2005) 117-132

23. Dubbelman M., Van der Heijde G.L., Weeber H.A., Vrensen G.F.J.M., Changes in the internal structure of the human crystalline lens with age and accommodation. Vision Res. 43 (2003) 2363-2375

24. Findl O., Drexler W., Menapace R., Hitzenberger C.K., Fercher A.F., High precision biometry of pseudophakic eyes using partial coherence interferometry. J Cataract Refract Surg. 24 (1998) 1087-1093

25. Foster P.J., Alsbirk P.H., Baasanhu J., Munkhbayar D., Uranchimeg D., Johnson G.J., Anterior chamber depth in Mongolians: variation with age, sex, and method of measurement. Am J Ophthalmol. 124 (1997) 53-60

26. Fukuyama M., Oshika T., Amano S., Yoshitomi F., Relationship between apparent accommodation and corneal multifocality in pseudophakic eyes. Ophthalmology. 106 (1999) 1178-1181

27. Gao L., Wang J., Zhuo X., Yu N., Ma L., Effect of ocular accommodation on refractive components in children. Yan Ke Xue Bao. 18 (2002) 208-213

28. Garner L.F., Smith G., Changes in equivalent and gradient refractive index of the crystalline lens with accommodation. Optom Vis Sci. 74 (1997) 114-119 
29. Garner L.F., Yap M.K.H., Changes in ocular dimensions and refraction with accommodation. Ophthal Physiol Opt. 17 (1997) 12-17

30. Gillum W., Mechanisms of accommodation in vertebrates. Ophthalmic Semin. 1 (1976) 253-286

31. Glasser A., Kaufman P.L., Accommodation and Presbyopia. In „Adler`s physiology of the eye", Kaufman P.L., Alm A. (Hrsg.), Mosby, Inc., St. Louis, 2003, 10. Auflage, $197-236$

32. Haefliger E., Parel J.M., Accommodation of an endocapsular silicone lens (PhacoErsatz) in the aging rhesus monkey. J Refract Corneal Surg. 10 (1994) 550-555

33. Haefliger E., Parel J.M., Fantes F., Norton E.W., Anderson D.R., Forster R.K., Hernandez E., Feuer W.J., Accommodation of an endocapsular silicone lens (PhacoErsatz) in the nonhuman primate. Ophthalmology. 94 (1987) 471-477

34. Haigis W., Pseudophakic correction factors for optical biometry. Graefes Arch Clin Exp Ophthalmol. 239 (2001) 589-598

35. Heatley C.J., Whitefield L.A., Hugkulstone C.E., Effect of pupil dilation on the accuracy of the IOLMaster. J Cataract Refract Surg. 28 (2002) 1993-1996

36. Heron G., Charman W.N., Accommodation as a function of age and the linearity of the response dynamics. Vision Res. 44 (2004) 3119-3130

37. Hitzenberger C.K., Optical Measurement of the axial eye length by laser doppler interferometry. Invest Ophthalmol Vis Sci. 32 (1991) 616-624

38. Ho A., Erickson P., Manns F., Pham T., Parel J.M., Theoretical analysis of accommodation amplitude and ametropia correction by varying refractive index in Phaco-Ersatz. Optom Vis Sci. 78 (2001) 405-410 
39. Hook D.W.A., Harding J.J., Physiology and biochemistry. In „Oxford Textbook of Ophthalmology", Easty D.L., Sparrow J.M.(Hrsg.), Oxford University Press, 1999, Volume 1, 456-463

40. Hosny M., Alio J.L., Claramonte P., Attia W.H., Perez-Santonja J.J., Relationship between anterior chamber depth, refractive state, corneal diameter, and axial length. $J$ Refract Surg. 16 (2000) 336-340

41. Hunter H. Jr, Shipp M., A study of racial differences in age at onset and progression of presbyopia. J Am Optom Assoc. 68 (1997) 171-177

42. Jimenez R., Gonzalez M.D., Perez M.A., Garcia J.A., Evolution of accommodative function and development of ocular movements in children. Ophthalmic Physiol Opt. 23 (2003) 97-107

43. Kasthurirangan S., Glasser A., Characteristics of pupil responses during far-to-near and near-to-far accommodation. Ophthalmic Physiol Opt. 25 (2005) 328-339

44. Katz M., The human eye as an optical system. In „Clinical Ophthalmology”, Duane, T.D. (Hrsg.), Harper\&Row Publishers, Phladelphia 1987, Volume 1, 1-52

45. Kielhorn I., Rajan M.S., Tesha P.M., Subryan V.R., Bell J.A., Clinical assessment of the Zeiss IOLMaster. J Cataract Refract Surg. 29 (2003) 518-522

46. Kirschkamp T., Dunne M., Barry J.C., Phakometric measurement of ocular surface radii of curvature, axial separations and alignment in relaxed and accommmodated human eyes. Ophthalmic Physiol Opt. 24 (2004) 65-73

47. Koeppl C., Findl O., Kriechbaum K., Drexler W., Comparison of pilocarpine-induced and stimulus-driven accommodation in phakic eyes. Exp Eye Res. 80 (2005) 795-800

48. Koretz J.F., Kaufman P.L., Neider M.W., Goeckner P.A., Accommodation and presbyopia in the human eye- aging of the anterior segment. Vision Res. 29 (1989) $1685-1692$ 
49. Koretz J.F., Cook C.A., Kaufman P.L., Accommodation and presbyopia in the human eye. Changes in the anterior segment and crystalline lens with focus. Invest Ophthalmol Vis Sci. 38 (1997) 569-578

50. Koretz J.F., Cook C.A., Kaufman P.L., Aging of the human lens: changes in lens shape at zero-diopter accommodation. J Opt Soc Am A Opt Image Sci Vis. 18 (2001) $265-272$

51. Koretz J.F., Handelman G.H., Brown N.P., Analysis of human crystalline lens curvature as a function of accommodative state and age. Vision Res. 24 (1984) 11411151

52. Kriechbaum K., Findl O., Kiss B., Sacu S., Petternel V., Drexler W., Comparison of anterior chamber depth measurement methods in phakic and pseudophakic eyes. $J$ Cataract Refract Surg. 29 (2003) 89-94

53. Kriechbaum K., Findl O., Koeppl C., Menapace R., Drexler W., Stimulus-driven versus pilocarpine-induced biometric changes in pseudophakic eyes. Ophthalmology. 112 (2005) 453-459

54. Küchle M., Nguyen N.X., Langenbucher A., Gusek- Schneider G.C., Seitz B., Zwei Jahre Erfahrung mit der akkommodativen Hinterkammerlinse 1 CU. Ophthalmologe. 11 (2002) 820-824

55. Kurtz D., Manny R., Hussein M., COMET Study Group, Variability of the ocular component measurements in children using A-scan ultrasonography. Optom Vis Sci. $81(2004) 35-43$

56. Lam A.K., Chan R., Pang P.C., The repeatability and accuracy of axial length and anterior chamber depth measurements from the IOLMaster. Ophthalmic Physiol Opt. 21 (2001) 477-483 
57. Langenbucher A., Reese S., Jakob C., Seitz B., Pseudophakic accommodation with translation lenses- dual optic vs. mono optic. Ophthalmic Physiol Opt. 24 (2004) 450457

58. Lawson M.L., Crewther S.G., Junghans B.M., Crewther D.P., Kiely P.M., Changes in ocular accommodation when shifting between global and local attention. Clin Exp Optom. 88 (2005) 28-32

59. Lesiewska- Junk H., Kaluzny J., Intraocular lens movement and accommodation in eyes of young patients. J Cataract Refract Surg. 26 (2000) 562-565

60. Lin J.T., Criteria for true accommodation and pseudo-accommodation caused by axial length elongation. J Refract Surg. 20 (2004) 397-398

61. Lin J.T., Oviedo F., New formulas comparing the accommodation in human lens and intraocular lens. J Refract Surg. 21 (2005) 200-201

62. Mallen E.A., Kashyap P., Hampson K.M., Transient axial change during the accommodation response in young adults. Invest Ophthalmol Vis Sci. 47 (2006) 12511254

63. Mastropasqua L., Toto L., Nubile M., Falconio G., Ballone E., Clinical study of the 1CU accommodating intraocular lens. J Cataract Refract Surg. 29 (2003) 1307-1312

64. McLeod S.D., Portney V., Ting A., A dual optic accommodating foldable intraocular lens. Br J Ophthalmol. 87 (2003) 1083-1085

65. Miller D., Optics and Refraction. In „Adler`s physiology of the eye", Kaufman P.L., Alm A. (Hrsg.), Mosby, Inc., St. Louis, 2003, 10. Auflage, 159-197

66. Mordi J.A., Ciuffreda K.J., Dynamic aspects of accommodation: age and presbyopia. Vision Res. 44 (2004) 591-601 
67. Moses R.A., Accommodation. In „Adler`s physiology of the eye", Moses RA, Hart WM (Hrsg.), c.v.Mosby Company, St. Louis, 1987, 8. Auflage, 291-310

68. Mundt G.H. Jr, Hughes W.F. Jr, Ultrasonics in ocular diagnosis. Am J Ophthalmol. 41 (1956), 488-498

69. Nakatsuka C., Hasebe S., Nonaka F., Ohtsuki N., Accommodative lag under habitual seeing conditions: comparison between myopic and emmetropic children. Jpn J Ophthalmol. 49 (2005) 189-194

70. Nawa Y., Ueda T., Nakatsuka M., Tsuji H., Marutani H., Hara Y., Uozato H., Accommodation obtained per $1.0 \mathrm{~mm}$ forward movement of a posterior chamber intraocular lens. J Cataract Refract Surg. 29 (2003) 2069-2072

71. Németh J., Fakete O., Pesztenlehrer N., Optical and ultrasound measurement of axial length and anterior chamber depth for intraocular lens power calculation. J Cataract Refract Surg. 29 (2003) 85-88

72. Nishi O., Nishi K., Accommodation amplitude after lens refilling with injectable silicone by sealing the capsule with a plug in primates. Arch Ophthalmol. 116 (1998) $1358-1361$

73. Ojaimi E., Rose K.A., Morgan I.G., Smith W., Martin F.J., Kifley A., Robaei D., Mitchell P., Distribution of ocular biometric parameters and refraction in a populationbased study of Australian children. Invest Ophthalmol Vis Sci. 46 (2005) 2748-2754

74. Ostrin L.A., Glasser A., Accommodation measurements in a prepresbyopic and presbyopic population, J Cataract Refract Surg. 30 (2004) 1435-1444

75. Parel J.M., Gelender H., Trefers W.F., Norton E.W., Phaco-Ersatz: cataract surgery designed to preserve accommodation. Graefes Arch Clin Exp Ophthalmol. 224 (1986) $165-173$ 
76. Parssinen O., Lyyra A.L., Myopia and moypic progression among schoolchildren: a three-year follow-up study. Invest Ophthalmol Vis Sci. 34(1993) 2794-2802

77. Quinn G.E., Francis E.L., Nipper K.S., Flitcroft D.I., Ying G.S., Rees R.C., Schmid G.F., Maguire M.G., Stone R.A., Highly precise eye length measurements in children aged 3 through 12 years. Arch Ophthalmol. 121 (2003) 985-990

78. Rager G., van der Zypen E., Auge. In „Anatomie. Makroskopische Anatomie, Embryologie und Histologie des Menschen/ Benninghoff", Benninghoff A.; Drenckhahn D., Zenker W. (Hrsg.), Urban und Schwarzenberg, München, Wien, Baltimore, 1993, 15. Auflage, Band 2, 701-752

79. Rohen J.W., Akkommodationsapparat. In „Der Augenarzt", Velhagen K. (Hrsg.), Georg Thieme Verlag, Leipzig, 1969, Band 1,2. Auflage, 51-69

80. Rohen J.W., Scanning electron microscopic studies of the zonular apparatus in human and monkey eyes. Invest Ophthalmol Vis Sci. 18 (1979) 133-144

81. Rosenfield M., Cohen A.S., Repeatability of clinical measurements of the amplitude of accommodation, Ophthalmic Physiol Opt. 16 (1996) 247-249

82. Rosenfield M., Gilmartin B., Accommodative error, adaptation and myopia, Ophthalmic Physiol Opt. 19 (1999), 159-164

83. Rosenfield M., Portello J.K., Blustein G.H., Jang C., Comparison of clinical techniques to assess the near accommodative response, Optom Vis Sci. 73 (1996) 382388

84. Sachsenweger M., Die Optik des Auges und ihre Störungen. In „Duale Reihe Augenheilkunde", Sachsenweger M.; Bob A., Bob K. (Hrsg.), Georg Thieme Verlag, Stuttgart 2003, 2. Auflage, 331-355 
85. Sachsenweger M., Lens cristallina. In „Duale Reihe Augenheilkunde”, Sachsenweger M.; Bob A., Bob K. (Hrsg.), Georg Thieme Verlag, Stuttgart 2003, 2. Auflage, 146171

86. Sachsenweger R., Friedburg D., Funktionsprüfung und Funktionsstörungen. In „Lehrbuch und Atlas der Augenheilkunde”, Axenfeld Th. (Begr.), Pau H. (Hrsg.), Gustav Fischer Verlag, Stuttgart, 1992, 13. Auflage, 29-34

87. Santodomingo- Rubido J., Mallen E.A., Gilmartin B., Wolffsohn J.S., A new noncontact optical device for ocular biometry. Br J Ophthalmol. 86 (2002) 458-462

88. Saw S.M., Chua W.H., Hong C.Y., Wu H.M., Chan W.Y., Chia K.S., Stone R.A., Tan D., Nearwork in early-onset myopia. Invest Ophthalmol Vis Sci. 43(2002) 332-339

89. Schaeffel F., Wilhelm H., Zrenner E., Inter-individual variability in the dynamics of natural accommodation in humans: relation to age and refractive errors. J Physiol. 461 (1993) 301-320

90. Schneider H., Stachs O., Gobel K., Guthoff R., Changes of the accommodative amplitude and the anterior chamber depth after implantation of an accommodative intraocular lens. Graefes Arch Clin Exp Ophthalmol. 244 (2006) 322-329

91. Seidel D., Gray L.S., Heron G., The effect of monocular and binocular viewing on the accommodation response to real targets in emmetropia and myopia. Optom Vis Sci. 82 (2005) 279-285

92. Shapiro J.A., Kelly J.E., Howland H.C., Accommodative status of young adults using reading spectacles. Vision Res. 45 (2005) 233-245

93. Sheng H., Bottjer C.A., Bullimore M.A., Ocular component measurement using the Zeiss IOLMaster. Optom Vis Sci. 81 (2004) 27-34

94. Shum P.J., Ko L.S., Ng C.L., Lin S.L., A biometric study of ocular changes during accommodation. Am J Ophthalmol. 115 (1993) 76-81 
95. Shun- Shin G.A., Anatomy of the lens. In „Oxford Textbook of Ophthalmology", Easty D.L., Sparrow J.M.(Hrsg.),Oxford University Press, 1999, Volume 1,453-456

96. Smith G., Atchison D.A., Pierscionek B.K., Modelling the power of the aging human eye. J Opt Soc Am A. 12 (1992) 2111-2117

97. Sterner B., Gellerstedt M., Sjöström A., The amplitude of accommodation in 6-10year-old children - not as good as expected! Ophthalmic Physiol Opt. 24 (2004) 246251

98. Stone R.A., Quinn G.E., Francis E.L., Ying G.S., Flitcroft D.I., Parekh P., Brown J., Orlow J., Schmid G., Diurnal axial length fluctuations in human eyes. Invest Ophthalmol Vis Sci. 45 (2004) 63-70

99. Strenk S.A., Semmlow J.L., Strenk L.M., Munoz P., Gronlund-Jacob J., DeMarco J.K., Age-related changes in human ciliary muscle and lens: a magnetic resonance imaging study. Invest Ophthalmol Vis Sci. 40(1999) 1162-1169

100. Tahi H., Fantes F., Hamaoui M., Parel J.M., Small peripheral anterior continuous curvilinear capsulorhexis. J Cataract Refract Surg. 25 (1999) 744-747

101. Tahi H., Fantes F., Hamaoui M., Parel J.M., Small peripheral anterior continuous curvilinear capsulorhexis. J Cataract Refract Surg. 25 (1999) 744-747

102. Tamm S., Tamm E., Rohen J.W., Age- related changes of the human ciliary muscle. A quantitative morphometric study. Mech Ageing Dev. 62 (1992) 209-221

103. Van der Heijde G.L., Beers A.P.A., Dubbelman M., Microfluctuations of steady-state accommodation measured with ultrasonography. Ophthal Physiol Opt. 16 (1996) 216221 
104. Vogel A., Dick H.B., Krummenauer F., Reproducibility of optical biometry using partial coherence interferometry: Intraobserver and interobserver reliability. $J$ Cataract Refract Surg. 27 (2001) 1961-1968

105. Weale R.A., Ageing changes in the lens. In „Oxford Textbook of Ophthalmology", Easty D.L., Sparrow J.M.(Hrsg.),Oxford University Press, 1999, Volume 1, 465-468

106. Weiss M., Seidemann A. Schaeffel F., Stimulating human accommodation without changes in focus. Ophthalmic Physiol Opt. 24 (2004) 207-217

107. Whitmore W.G., Comments to: A biometric study of ocular changes during accommodation. Am J Ophthalmol. 115 (1993) 682

108. Wick B., Gall R., Yothers T., Clinical testing of accommodative facility: part III. Masked assessment of the relation between visual symptoms and binocular test results in school children and adults. Optometry. 73 (2002) 173-181

109. Wick B., Yothers T.L., Jiang B.C., Morse S.E., Clinical testing of accommodative facility: Part I. A critical appraisal of the literature. Optometry. 73 (2002) 11-23

110. Wold J.E., Hu A., Chen S., Glasser A., Subjective and objective measurement of human accommodative amplitude. J Cataract Refract Surg. 29 (2003) 1878-1888

111. Wong T.Y., Foster P.J., Johnson G.J., Klein B.E., Seah S.K., The relationship between ocular dimensions and refraction with adult stature: The Tanjong Pagar survey. Invest Ophthalmol Vis Sci. 42 (2001)1237-1242

112. Yothers T., Wick B., Morse S.E., Clinical testing of accommodative facility: part II. Development of an amplitude-scaled test. Optometry. 73 (2002) 91-102

113. Zadnik K., Mutti D.O., Adams A.J., The repeatability of measurement of the ocular components. Invest Ophthalmol Vis Sci. 33 (1992) 2325-2333 


\title{
Danksagung
}

\author{
Prof. Dr. med. Ines Lanzl
}

für die Überlassung des interessanten Themas, große Unterstützung und hilfreiche Anregungen

\section{PhD Konstantin Kotliar}

für die kompetente, herausragende und stets bereichernde Betreuung

gesamtes Team des Augen-MVZ Landshut,

Dr. med. Bernhard Kölbl, Prof. Dr. med. Matthias Sachsenweger, Dr. med. Josef Reiter und Kollegen für die herzliche Aufnahme, Bereitstellung der Geräte und Räume, Rekrutierung der

Probanden, Interesse und Anregungen

Frau Regina Hollweck (IMSE TUM)

für ihre Geduld und die Anregungen

Dr. med. Dubravka und Axel Cartsburg

für die bedingungslose Unterstützung

Georg Anetsberger

für hilfreiche Diskussionen

meiner Familie,

der diese Arbeit gewidmet ist

Studienstiftung des deutschen Volkes

für Förderung und Unterstützung all meiner Vorhaben

allen Freunden und Probanden,

die an den umfangreichen Messungen teilgenommen und somit diese Arbeit erst möglich gemacht haben, gilt mein besonderer Dank 


\section{Appendix: Publikation „Messung der Akkommodation mittels optischer}

\section{Biometrie“}

Der Artikel in diesem Anhang wurde online in „Der Ophthalmologe“ publiziert,

Ophthalmologe 2007 DOI 10.1007/s00347-007-1622-x, Springer Medizin Verlag 2007 
Ophthalmologe 2007

DOI 10.1007/s00347-007-1622-x

C) Springer Medizin Verlag 2007
A. Nurispahic · K. Kotliar - I. Lanzl

Augenklinik der TU München

\section{Messung der Akkommodation mittels optischer Biometrie}

\section{Hintergrund}

Die Fähigkeit zur Akkommodation reduziert sich bereits im frühen Lebensalter, sodass beim ca. 40-Jährigen eine Lesebrille erforderlich wird. Akkommodation auch im Alter zu ermöglichen ist Ziel vieler Forschungsvorhaben, der Erfolg bislang noch umstritten. Die genaue Kenntnis des physiologischen Verhaltens einzelner Augenabschnitte und ihrer Ånderungen während der Naheinstellung stellen das notwendige Fundament dar, um Uberlegungen zur Rekonstruktion anzustellen [4].

In der Vielzahl der in den letzten Jahren zum Thema Akkommodation erschienenen Arbeiten werden unterschiedlichste Stimulations- und Messmethoden eingesetzt. Neben pharmakologischer Stimulation mit Pilokarpin, die jedoch in der Gruppe der Pseudophaken inzwischen als Superstimulus gilt [13], kamen auch objektive und subjektive physiologische Techniken zum Einsatz $[23,31]$. Bei den Messungen wurden die Scheimpflug-Fotografie [14], die optische Kohärenztomographie [2] und Ultraschall $[3,8]$ angewandt. In letzter Zeit wurde der IOLMaster als nichtinvasives Messgerät häufig eingesetzt. Dabei konnten die Genauigkeit und Reproduzierbarkeit seiner Messmethoden bestätigt werden $[5,19,26]$.

Die einzelnen Faktoren, die zur Presbyopie [14] führen, sowie der Zusammenhang zwischen Akkommodationsantwort und unterschiedlichen Symptomen [30] und von den Verhältnissen innerhalb des Auges und ihren Schwankungen im Tagesverlauf [28] werden in zahlreichen Studien z. T. kontrovers diskutiert. Neben der Zunahme der Achsenlänge während der
Naheinstellung $[7,27]$, bestehen auch über die Akkommodationsfähigkeit und Position von Intraokularlinsen unterschiedliche Ansichten [18, 25]

Aufgrund der verschiedenen, teils widersprüchlichen Beobachtungen in der Fachliteratur hatte diese Arbeit das Ziel, einen objektiven Beitrag zuden Bemühungen zu leisten, Akkommodation zu verstehen und evtl. zu rekonstruieren. Untersuch wurden die Längenverhältnisse in der Sehachse, Vorderkammertiefe (VKT) und Achsenlänge (AL) während der Akkommodation bei verschiedenen Altersgruppen und zusätzlich bei pseudophaken Probanden. Vor allem die Änderung dieser Parameter bei phaken Probanden während physiologisch stimulierter Akkommodation sowie die Unterschiede in der Ånderung der VKT bei Pseudophaken im Vergleich zu einer altersgematchten phaken Erwachsenengruppe waren von Interesse.

\section{Materialien und Methoden}

Materialien und Probanden

Die Messungen wurden mittels IOL-Master (Zeiss/Jena) durchgeführt. Beim IOL-Master handelt es sich um ein kombiniertes Biometriegerät, das bei der Erfassung der VKT auf die konventionelle Spaltlampentechnik

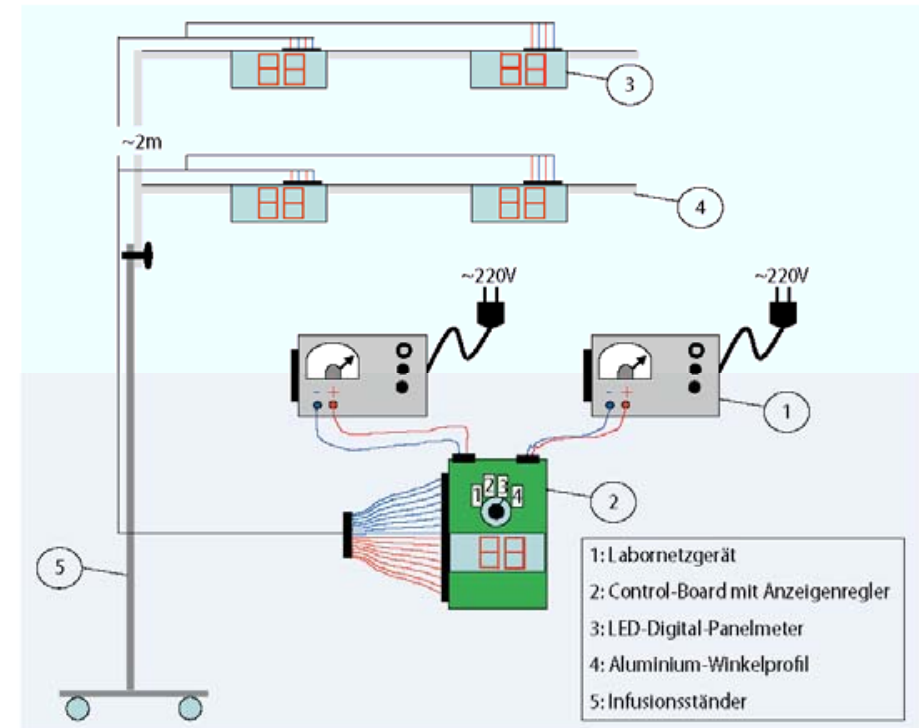

Abb. 1 A Zusatzeinrichtung zur physiologischen Stimulation der Akkommodation, Schema 


\section{Originalien}

Tab. 1 Vorderkammertiefe (VKT) und Achsenlänge (AL) im unstimulierten Zustand

\begin{tabular}{lllllll} 
Untersuchungsgruppen & VKT $(\mathrm{mm})$ & & & AL $(\mathrm{mm})$ & & \\
& MW \pm SA & Max & Min & MW \pm SA & Max & Min \\
Kinder & $3,62 \pm 0,19$ & 3,98 & 3,33 & $23,19 \pm 0,49$ & 24,35 & 22,45 \\
\hline Jugendliche & $3,76 \pm 0,23$ & 4,21 & 3,21 & $23,94 \pm 0,79$ & 25,35 & 22,46 \\
Erwachsene & $3,21 \pm 0,33$ & 3,95 & 2,63 & $23,66 \pm 0,73$ & 25,33 & 22,63 \\
Pseudophake & $3,90 \pm 0,20$ & 4,17 & 3,46 & $23,05 \pm 0,79$ & 24,25 & 21,44
\end{tabular}

Tab. 2 Änderung der VKT und AL bei Nahakkommodation

\begin{tabular}{|c|c|c|c|c|c|c|c|c|}
\hline \multirow[t]{2}{*}{$\begin{array}{l}\text { Untersuchungs- } \\
\text { gruppen }\end{array}$} & \multicolumn{4}{|c|}{$\begin{array}{l}\text { Differenz zum unstimulierten } \\
\text { Zustand } \triangle \mathrm{VKT}(\mathrm{mm})\end{array}$} & \multicolumn{4}{|c|}{$\begin{array}{l}\text { Differenz zum unstimulierten } \\
\text { Zustand } \triangle A L(\mathrm{~mm})\end{array}$} \\
\hline & $M W \pm S A$ & Max & Min & $\begin{array}{l}\text { Signifi- } \\
\text { kanz }\end{array}$ & $\mathrm{MW} \pm \mathrm{SA}$ & Max & Min & $\begin{array}{l}\text { Signifi- } \\
\text { kanz }\end{array}$ \\
\hline Kinder & $0,11 \pm 0,03$ & 0,16 & 0,07 & $p<0,01$ & $0,01 \pm 0,01$ & 0,02 & 0,00 & $p<0,01$ \\
\hline Jugendliche & $0,14 \pm 0,03$ & 0,20 & 0,06 & $p=0,00$ & $0,01 \pm 0,01$ & 0,04 & 0,00 & $p<0,01$ \\
\hline Erwachsene & $0,07 \pm 0,02$ & 0,11 & 0,04 & $p<0,01$ & $0,01 \pm 0,01$ & 0,02 & 0,00 & $p<0,01$ \\
\hline Pseudophake & $0,00 \pm 0,00$ & 0,01 & 0,00 & $p>0,05$ & $0,01 \pm 0,01$ & 0,01 & 0,00 & $p<0,01$ \\
\hline
\end{tabular}

\begin{tabular}{|c|c|c|c|c|c|}
\hline \multirow[t]{2}{*}{ Gruppe } & & \multicolumn{2}{|l|}{ VKT (mm) } & \multicolumn{2}{|l|}{$\mathrm{AL}(\mathrm{mm})$} \\
\hline & & $\begin{array}{l}\text { Nahakkommo- } \\
\text { dation }\end{array}$ & $\begin{array}{l}\text { Fernakkom- } \\
\text { modation }\end{array}$ & $\begin{array}{l}\text { Nahakkommo- } \\
\text { dation }\end{array}$ & $\begin{array}{l}\text { Fernakkommo- } \\
\text { dation }\end{array}$ \\
\hline \multirow[t]{3}{*}{ Kinder } & $M W \pm S A$ & $3,51 \pm 0,20$ & $3,63 \pm 0,19$ & $23,19 \pm 0,49$ & $23,19 \pm 0,49$ \\
\hline & $\operatorname{Max}$ & 3,91 & 3,98 & 24,35 & 24,35 \\
\hline & Min & 3,22 & 3,34 & 22,46 & 22,45 \\
\hline \multirow[t]{3}{*}{ Jugendliche } & $M W \pm S A$ & $3,62 \pm 0,23$ & $3,76 \pm 0,23$ & $23,95 \pm 0,79$ & $23,94 \pm 0,79$ \\
\hline & $\operatorname{Max}$ & 4,09 & 4,21 & 25,37 & 25,35 \\
\hline & Min & 3,05 & 3,21 & 22,47 & 22,46 \\
\hline \multirow[t]{3}{*}{ Erwachsene } & $M W \pm S A$ & $3,15 \pm 0,34$ & $3,22 \pm 0,33$ & $23,67 \pm 0,73$ & $23,66 \pm 0,73$ \\
\hline & $\operatorname{Max}$ & 3,90 & 3,95 & 25,34 & 25,33 \\
\hline & Min & 2,53 & 2,64 & 22,63 & 22,63 \\
\hline \multirow[t]{3}{*}{ Pseudophake } & $M W \pm S A$ & $3,90 \pm 0,20$ & $3,90 \pm 0,19$ & $23,05 \pm 0,79$ & $23,04 \pm 0,79$ \\
\hline & $\operatorname{Max}$ & 4,16 & 4,17 & 24,26 & 24,25 \\
\hline & Min & 3,46 & 3,47 & 21,45 & 21,44 \\
\hline
\end{tabular}

zurückgreift. Beider AL-Messung hingegen kommt die PCI (partielle Kohärenzinterferometrie) zum Einsatz. Ihr Prinzip wurde in anderen Publikationen ausführlich erklärt [24]. Wie bei der PCI im Allgemeinen müssen auch beim IOL-Master die gemessenen optischen Strecken in geometrische Längen umgewandelt werden, was in diesem Fall auf der Grundlage des Augenmodells nach Gullstrand erfolgt [1].

An der Studie nahmen 6o Probanden teil. Diese wurden in 4 verschiedene Gruppen von jeweils 15 Personen eingeteilt: Kinder (10,5 $\pm 1,9$ Jahre), Jugendliche $(21,9 \pm 2,1$ Jahre), Erwachsene ( $55,7 \pm 7,3$ Jahre) und etwa vergleichbar alte pseudophake Erwachsene ( $59,9 \pm 7,8$ Jahre). Für die ersten 3 Probandengruppen galten folgende Ausschlusskriterien:

- Refraktionsanomalie von mehr als 1,8 dpt,
- Astigmatismus von mehr als 0,5 dpt, - Anisometropie von mehr als 1,o dpt,

- Entzündungen am Auge,

- Amblyopie,

- Strabismus,

- vorangegangene Applikation von Medikamenten sowie

- ein chirurgischer Eingriff am Auge.

Die Gruppe der Pseudophaken [ $27 \%(n=4)$ Silikonlinsen- und $73 \%(\mathrm{n}=11)$ Acryllin senträger, jeweils 3-piece-Modelle] hatte natürlich bereits eine oder mehrere Operationen am Auge erfahren.

\section{Methoden}

Die Stimulation der Akkommodation ereinrichtung (- Abb. 1, 2). Uber dem Probanden wurden im Abstand von 16 und folgte physiologisch mittels einer Zusatz-
$216 \mathrm{~cm}$ jeweils 2 Digitalanzeigen über dem rechten und dem linken Auge angebracht, die zur Anregung der Nah- bzw. Fernakkommodation dienen sollten. Diese konnten Zahlen von oo-99 anzeigen, wobei die aktuelle Zahl beliebig vom Untersucher eingestellt werden konnte, indem die Spannung der Energiequelle verändert wurde. Gemessen wurde die ipsilaterale Akkommodationsantwort. Da zu wurde mittels Fixationsschienen am Gerüst des IOL-Masters ein halbdurchlässiges Glasplättchen befestigt. Dieses konnte sich der Proband so vor dem Auge einstellen, dass darin das Spiegelbild der oben eingeblendeten Ziffern gut zu erkennen war. Die Einspiegelung erfolgte dabei so, dass sich das Fixierlicht des IOL Masters etwa in der Mitte zwischen den beiden Ziffern befand.

Das rechte Auge der Probanden wurde untersucht, bei unilateral Pseudopha ken entsprechend das Auge mit der implantierten Kunstlinse.

Die Planung und Durchführung die ser Arbeit erfolgte unter Berücksichtigung der Deklaration von Helsinki nach dem Votum der Ethikkommission der TU München. Die Messungen erfolgten erst nach einer ausführlichen Anamne se, Aufklärung und Einholung einer Einverständniserklärung der Probanden bzw. der Erziehungsberechtigten. Der vom Hersteller vorgeschriebene Grenzwert von $20 \mathrm{AL}$-Messungen pro Tag und $\mathrm{Au}$ ge wurde nicht überschritten. Zunächst wurden beide Augen im unstimulierten Zustand ausgemessen (AL, Hornhaut krümmung, VKT). Anschließend wurde zunächst der fernere, dann der nahe Akkommodationsreiz präsentiert, und $\mathrm{AL}$ sowie VKT-Messungen wurden durchgeführt. Mit Ausnahme der Kindergruppe erfolgten alle Messungen der AL und der VKT jeweils 4-mal, wobei im Weiteren die Mittelwerte vorgestellt werden. Bei Kindern wurde die Anzahl der Messungen auf 2 beschränkt und damit der Tatsache Rechnung getragen, dass ihre Konzentration leichter nachließ.

Beide angewandten Methoden wurden im Vergleich zwischen 2 Untersu chern auf Reproduzierbarke it überprüft. Dabei wurden 5 emmetrope jugendliche Probanden in einem randomisierten Verfahren an beiden Augen im unakkom

2 Der Ophthalmologe 2007 
Zusammenfassung · Abstract

modierten und nahakkommodierten $\mathrm{Zu}$ stand vermessen.

Die Datenanalyse und Statistik wurde unter Anwendung der Programme "Microsoft Excel" und „SPSS, Version $13.0^{\circ}$ durchgeführt.

Die deskriptive statistische Auswertung erfolgte bei quantitativen Größen anhand von Mittelwert (MW), Standardabweichung (SA), und ausgesuchten Quantilen (Min, Median, Max), bei qualitativen Merkmalen wurde die Beschreibung mithilfe der Häufigkeitsverteilung durchgeführt. Die Ergebnisse wurden überdies auf Signifikanz getestet, wobei $\mathrm{p}<0,05$ als signifikant gedeutet wurde. Für Veränderungen innerhalb einer Augendimension, also der VKT bzw. der AL, wurde ein 2seitiger, gepaarter t-Test verwendet. Bei Mehrfachvergleichen zwischen den Gruppen wurde der Vielzahl der Vergleiche durch die Oneway-ANOVA-Analyse mit dem Post-hoc-Bonferroni-Test Rechnung getragen. Zur Prüfung der bivariaten Korrelation zwischen den Methoden und den beiden Untersuchern wurde der Rangkorrelationskoeffizient von Pearson verwendet, wobei ein 2-seitiges Signifikanzniveau von 0,01 galt.

Den Ergebnissen sei vorangestellt, dass sich im Vergleich zwischen den beiden Untersuchern signifikant $(\mathrm{p}<\mathrm{O}, \mathrm{O} 1)$ hohe Korrelationen $(r>0,97)$ sowohl bei Messungen der VKT als auch bei jenen der ALergaben.

\section{Ergebnisse}

\section{Statische Messungen: VKT und AL}

Die im unstimulierten Zustand gemessenen Werte der VKT und AL werden in - Tab. 1 zusammengefasst.

Im Mehrfachvergleich der verschiedenen Gruppen stellte sich heraus, dass sich die VKT im unstimulierten $\mathrm{Zu}$ stand bei Kindern zwar nicht signifikant von jener bei Jugendlichen unterschieden $(p=0,73)$, wohingegen sich bereits zwischen Jugendlichen und Erwachsenen signifikante $(\mathrm{p}<0,01)$ Unterschiede bei der Tiefe der Vorderkammer nachweisen ließen. Einen Uberblick über die Verteilung der VKT im unstimulierten Zustand bei den verschiedenen Gruppen gibt $\mathbf{A b b} 3$.
Ophthalmologe 2007 DOI 10.1007/500347-007-1622-x

O Springer Medizin Verlag 2007

\section{A. Nurispahic $\cdot$ K. Kotliar - I. Lanzl}

Messung der Akkommodation mittels optischer Biometrie
Zusammenfassung

Hintergrund. Um Akkommodation auch im Alter ermöglichen zu können, ist es wichtig, die dabei stattfindenden Vorgänge im Detail zu verstehen. In dieser Arbeit wurden Länkommodation bei unterschiedlichen Altersgruppen und Linsenstatus untersucht. Methoden. Bel 60 Probanden (Kinder, Jugendliche, Erwachsene, Pseudophake) wurden Vorderkammertiefe (VKT) und Augenlänge (AL), sowle ihre Änderung bei physiologisch ausgelöster Akkommodation mit dem IOL-Master ermittelt.

Ergebnisse. Dle VKT nahm mit dem Alter ab. Die größten Verkürzungen der VKT bei Nahgenverhältnisse in der Sehachse während Ak- akkommodation (NA) ergaben sich bei Jugendlichen $(0,14 \pm 0,03 \mathrm{~mm})$. Wahrend in der Erwachsenengruppe die VKT bei NA abnahm, trat dies bei Pseudophaken nicht ein. Die AL nahm bei allen Gruppen während NA minimal zu $(0,01 \pm 0,01 \mathrm{~mm})$.

Diskussion. Mittels physiologischer Stimulation war bel Pseudophaken kaumeine Änderung der VKT bei NA messbar. Die gemessene Verlängerung der AL ist genauer zu untersuchen.

Schlüsselwörter

Akkommodation - Vorderkammertiefe - Alter Achsenlänge $\cdot$ IOL-Master - Biometrie

\section{Measurement of accommodation using optical biometry}

\section{Abstract}

Background. Making accommodation possible for all age groups is a topic of great interest. We applied optical biometry in order to study the physiological mechanisms in detail. Longitudinal relations in the optical axis were measured during accommodation in volunteers of different ages and lens states. Methods. A total of 60 subjects (children, adolescents, adults, and pseudophakes) were examined using the IOL Master. We measured anterior chamber depth $(A C D)$, axial length (AL), and changes in these two measurements during accommodation.

Results. Near accommodation (NA) in adolescents caused the largest ACD decrease
$(0.14 \pm 0.03 \mathrm{~mm})$. ACD decreased in adults during NA but not in pseudophakic patients of comparable age. AL increased during NA in all groups by $0.01 \pm 0.01 \mathrm{~mm}$.

Condusions $A C D$ decreased with age. Using a physiological stimulus, no change in ACD was measured during NA in pseudophakic patients. The documented increase in AL needs to be evaluated further.

Keywords

Accommodation - Anterior chamber depth, age $\cdot$ Axial length $\cdot 1 \mathrm{OL}$ Master $\cdot$ Biometry 


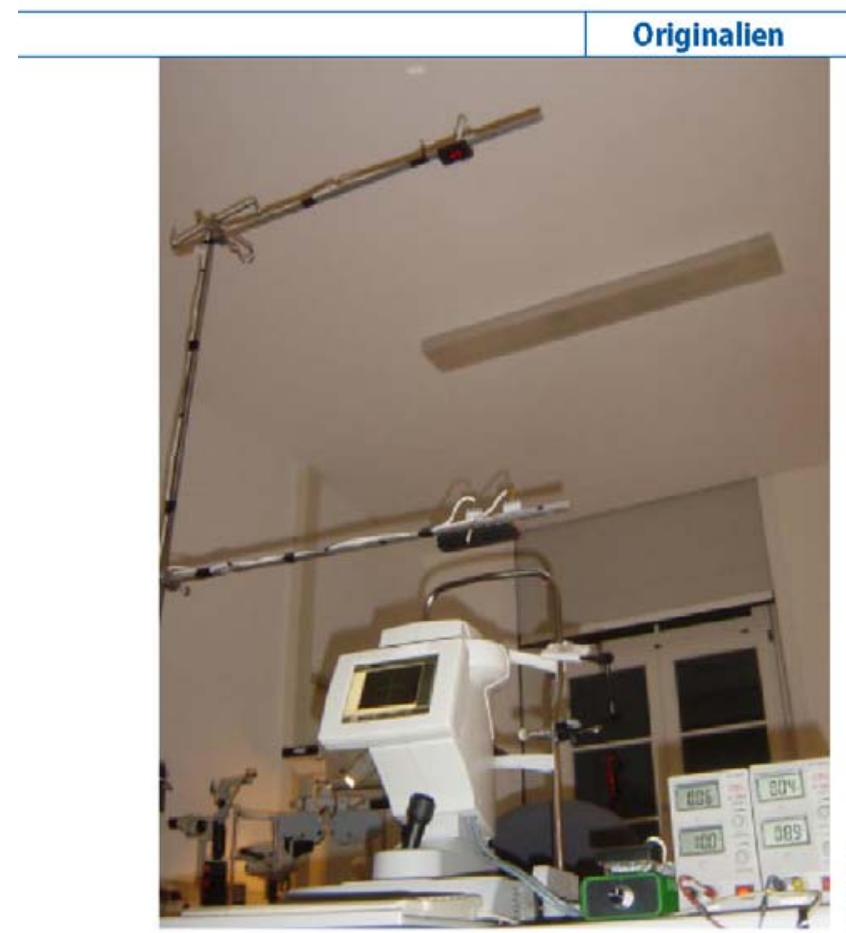

Abb. $2 \varangle$ Fotografische Abbildung der Zusatzeinrichtung am IOL-Master

Während sich bei der VKT signifikante Unterschiede mit dem Alter ergaben, konnten bei der AL zwischen Jugendlichen und Erwachsenen keine signifikanten Unterschiede im unstimulierten Zustand nachgewiesen werden $(\mathrm{p}=0,42)$.

Änderung der Parameter VKT und $A L$ bei der Akkommodation

Bei der Nahakkommodation ergaben sich deutliche Änderungen der VKT im Vergleich zum unstimulierten Zustand (- Tab. 2, $\bullet$ Abb. 4).

Sowohl bei Kindern als auch bei Jugendlichen und Erwachsenen wurde eine deutliche Verkürzung der VKT bei Nahakkommodation im Vergleich zur Messung ohne Akkommodationsreiz beobachtet. In diesem Punkt unterschieden sich die Pseudophaken deutlich von den anderen 3 Gruppen. Bei der Nahakkommodation konnte in ihrer Gruppe in der Mehrzahl der Fälle $(80 \%, n=12)$ keinerlei Veränderung der VKT im Vergleich zum unstimulierten Zustand gemessen werden. Lediglich bei $20 \%(n=3)$ der Pseudophaken ließ sich eine Verkürzung der VKT um o,o1 mm messen. Ebenso wenig ließ sich in dieser Gruppe statistisch eine signifikante Ånderung festmachen ( $\mathrm{p}=0,08$ ).

Die Ergebnisse der Messungen wäh schieden sich nicht signifikant von jenen im unstimulierten Zustand. Die absolut erhobenen Werte für VKT und AL sind in Tab. 3 aufgeführt.

\section{Diskussion}

Aus Publikationen anderer Arbeitsgruppen war bereits bekannt, dass der IOL-Master untersucherunabhängige Engebnisse liefert [10]. Dies konnte auch in unserer Arbeit bestätigt werden. Darüber hinaus konnten wir mit unserem Versuchsaufbau Akkommo dation verlässlich stimulieren.

\section{VKT und AL im}

\section{unstimulierten Zustand}

Wir beobachteten, dass die VKT mit dem Alter abnimmt. Eine Abflachung der Vorderkammer bei Erwachsenen ist durch $\mathrm{Zu}$ nahme der Linsendicke im Rahmen des Al terungsprozesses und die Vorverlagerung der Linsenmasse nach vorne auch zu erwarten [15]. Die Genauigkeit der mit dem IOL Master gemachten VKT-Messungen in der rend der Fernakkommodation unter
Gruppe der Pseudophaken ist sicherlich zu hinterfragen. Es ist aus vorangegangenen Studien bereits bekannt, dass im Gegensatz zur Messung der AL die Ergebnisse der Untersuchungen der VKT zwischen Ultraschall und IOL-Master in diesem Probandenkollektiv nur gering korrelieren [21]. Aufgrund unterschiedlicher physikalischer Messprinzipien haben die beiden Methoden unterschiedliche Bezugs- und Definitionspunkte. Es ist überdies nicht auszuschließen, dass der IOL-Master in manchen Fällen von falschen Bezugspunkten, wie z. B. der Iris oder der Linsenrückfläche, ausgeht. Trotzdem wird das Gerät im klinischen Alltag zur Prüfung der exakten Linsenposition auch nach Kataraktoperationen eingesetzt. Der IOL-Master kann v. a. bei sehr kleinen Pupillen, tiefen Vorderkammern oder bestimmten Haptiken die VKT nicht ermitteln bzw. orientiert sich z. B. an der Iris, wodurch sich falsche, kurze VKT ergeben [16]. Bei den hier vorgestellten Probanden konnten die Messungen jedoch problemlos durchgeführt werden. Eine mögliche Fehlorientierung an der Linsenrückfläche ist für unsere Messungen nicht relevant, da wir Änderung der VKT untersuchten und von einer konstanten Dicke der IOL im unstimulierten und im akkommodierten Zustand ausgehen.

Bei Messungen der AL wurde bei Pseudophaken der jeweilige Linsentyp am Gerät eingestellt und so automatisch der Korrekturfaktor mitberücksichtigt. In allen 4 Gruppen waren schnelle, präzise und konstante Messungen der AL möglich. Eine Änderung der AL mit dem Alterungsprozess wurde nicht beobachtet.

Änderung der AL und VKT durch Akkomodation bei phaken Probanden

Besonders interessant ist sicherlich die Frage, wie sich die Parameter VKT und AL bei Nahakkommodation verändern.

Was die Ånderung der AL bei Nahakkommodation betrifft, so wurden ähnliche Ergebnisse in allen 4 Gruppen erhoben. Bei der Mehrzahl der Probanden wurde eine signifikante Zunahme der AL um durchschnittlich $0,01 \mathrm{~mm}$ beobachtet. Da dieser Wert an die Genauigkeitsgrenze des IOLMasters stößt und kleinste Änderungen der Messachse, die es bei minimalen Augenro-

\section{Der Ophthalmologe 2007}


tationen geben könnte, nicht vollständig auszuschließen sind [11], gilt es, dieses Ergebnis weiter zu hinterfragen. Es ist auch bekannt, dass die AL tagesabhängigen Schwankungen unterliegt sowie Fluktuationen im Bereich von 0,002-0,01 mm allein mit Variationen der Herzfrequenz und der Atmung auftreten können [29]. Darüber hinaus könnte die Messmethode des IOLMasters selbst eine Erklärung bieten. Tatsächlich misst der IOL-Master nämlich die optische Achsenlänge des Auges. Da es bei Akkommodation zu einer Verdickung der Linse und somit zu einer Erhöhung des Brechungsindexes kommt, kann es selbst bei gleich gebliebener tatsächlicher $\mathrm{AL}$ zu einem systematischen Fehler bei der Umrechnung kommen, der je nach Stärke der Akkommodation bis hin zu o, $018 \mathrm{~mm}$ ausmachen kann [1]. Wir untersuchten jedoch auch pseudophake Patienten, bei denen die IOL keine Veränderung des Brechungsindexes erfahren konnte. Auch in dieser Gruppe wurde z T. eine Veränderung der AL beobachtet, was sich durch optische Phänomene im Messprinzip des IOL-Masters nicht erklären lässt.

Es ist daher zu überlegen, wie diese beobachteten Änderungen im Mikrometerbereich anders erklärt werden können. Einige Arbeitsgruppen schlagen vor, dass die mit der Akkommodation einhergehende Kontraktion des Ziliarmuskels zu einer Änderung der skleralen Form führt, indem sie an der Choroidea einen nach vorne innen gerichteten Zug ausübt [6]. Weiterhin ist es möglich, dass der bei der Adduktion im Rahmen der Konvergenzreaktion durch die extraokularen Muskeln ausgeübte Zug ebenfalls eine Verformung des Bulbus zur Folge hat. Dies könnte ebenfalls die Erklärung für die minimale Zunahme der $\mathrm{AL}$ bei Nahakkommodation bieten. Dennoch gilt es zu unterstreichen, dass bei einer gemessenen Änderung von 0,1 mm von einer damit einhergehenden Refraktionsänderung im Größenordnungsbereich von 0,3 dpt auszugehen ist, womit kein ausreichender Nahvisus gewährleistet wäre.

Bei der VKT ergaben sich während der Nahakkommodation deutliche Unterschiede zwischen den Gruppen. Die größten Abflachungen der Vorderkammer wurden bei Jugendlichen und Kindern gemessen. Nach der allgemein anerkannten Theorie von Helmholtz beruht

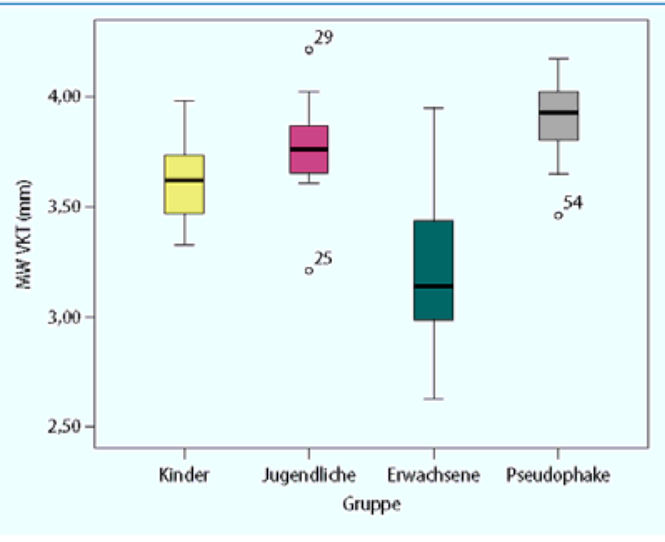

Abb. 3> Boxplot zu Verteilung derVKT im unstimulierten $\mathrm{Zu}$ stand, Gruppenvergleich

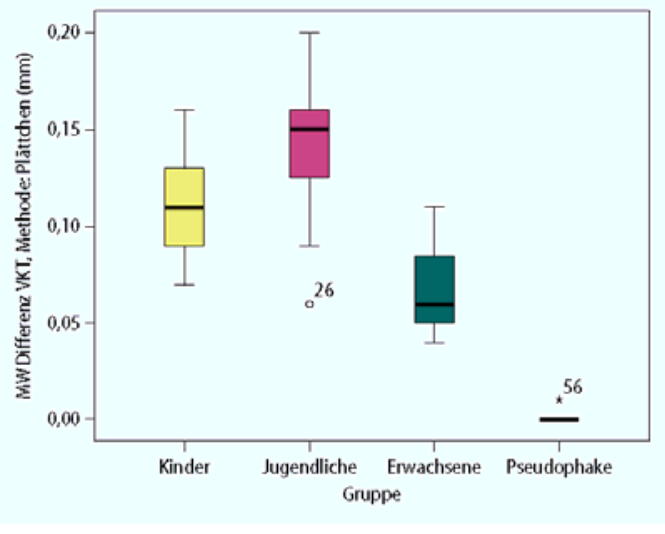

bb. $4 \triangleright$ Boxplot zur Differenz VKT, Gruppenvergleich

dieser Effekt auf einer Anspannung des Ziliarmuskels, die zu einer Entspannung der Zonulafasern führt. So kann die Lin senkapsel die Linse in eine rundere Form drücken [22]. Der anteriore Linsenpol be wegt sich dabei nach vorne, was eine $\mathrm{Ab}$ nahme der VKT zur Folge hat.

$\mathrm{Da}$ zu erwarten ist, dass bei emmetropen Kindern die Akkommodationsfähig keit größer ist als bei emmetropen Jugend. lichen, erstaunt die Beobachtung, dass die VKT-Änderung bei Nahakkommodation in der Kindergruppe im Durchschnitt et was kleiner ausfiel als bei Jugendlichen Naheliegend ist sicherlich die Erklärung, dass bei einem Nahstimulus in $16 \mathrm{~cm}$ Entfernung die Akkommodation bei Kindern noch nicht maximal angeregt wurde. Bei Betrachtung der zu Kindern vorhandenen Literatur werden darüber hinaus viele an dere Faktoren diskutiert. So wird z. B. vor geschlagen, grundsätzlich zwischen Kin dern im Schulalter und jüngeren zu un terscheiden [9]. Auch die Auffassungsga be wird als möglicher Grund angeführt [30]. Die an dieser Studie teilnehmenden Kinder waren jedoch in der Lage, Aufforderungen zuverlässig zu folgen. Von Vorohne subjektive Angaben auskommen konnte, was bei anderen Studien häufig als Fehlerquelle diskutiert wurde.

\section{Änderung derVKT durch Akkomodation bei Pseudophaken im Vergleich zu phaken Erwachsenen}

Deutlich kleinere Änderungen der VKT im Vergleich zu Kindern und Jugend lichen wurden bei Erwachsenen verzeichnet, wobei hier selbst innerhalb der Grup pe starke altersabhängige Unterschiede beobachtet wurden: Die maximale Abflachung der VKT bei Nahakkommodation wurde bei einer 48 -jährigen Person geteil war überdies, dass die Messanordnung 


\section{Originalien}

messen. Die Minima von 0,04 mm jedoch ergaben sich bei den Ältesten der Gruppe (66 und 69 Jahre). Aus vorangegangenen Studien ist bereits bekannt, dass die Akkommodationsbreite v. a. zwischen 30 und so Jahren abnimmt, während sich in den darauf folgenden Jahren die Akkommodationsfähigkeit vergleichsweise geringer ändert [12]. Dennoch unterschied sich diese Gruppe entscheidend von vergleichbar alten Pseudophaken.

Die Frage, ob in der Gruppe der Pseudophaken neben der „Pseudoakkommodation" auch eine echte Akkommodation stattfindet, die durch die Interaktion zwischen dem sich kontrahierenden Ziliarmuskel und der Kunstlinse zustande kommt, ist immer noch heftig umstritten. In dieser Arbeit konnte bei der Mehrzahl der pseudophaken Probanden mit physiologischem Reiz keinerlei Ånderung der VKT bei Nahakkommodation gemessen werden. Dafür kommen verschiedene Gründe infrage. Denkbar wäre, dass der im Alter zwar noch funktionsfähige Ziliarmuskel zu schwach ist, um die IOL zu bewegen [17], bzw. dass er sich zwar gut kontrahiert, die Bewegung dennoch insuffizient auf die IOL übertragen wird. Der ausgeübte Zug des Ziliarmuskels hat im physiologischen Zustand eine Verformung der Linsenkapsel als Ziel und nicht eine Vorwärtsbewegung des Kapselsacks mitsamt herkömmlicher IOL. Der von uns benutzte akkommodative Stimulus war jedoch in den anderen 3 Gruppen ausreichend groß, um eine Abflachung der VKT hervorzurufen. Diese Beobachtung bestätigt Erkenntnisse aus anderen Studien [17]. Andere Autoren fanden wiederum bei jungen pseudophaken Patienten Abflachungen der VKT in der Größenordnung von $0,42 \mathrm{~mm}$ bei Nahakkommodation [20]. Aus den Daten unserer Arbeit ergibt sich, dass eine echte $\Lambda$ kkommodation bei Pseudophaken mit herkömmlichen monofokalen Linsen durch Verschiebung der Linsenposition im Auge beiAkkommodationsanstrengung nicht nachzuweisen ist.

\section{Fazit für die Praxis}

Der IOL-Master eignet sich unter sorgfältiger Berücksichtigung von Störfaktoren auch für die Messung derVorderkammertiefe bei Pseudophaken. Die größten Abnahmen derVKT bei physiologisch sti- mulierter Nahakkommodation sind be Jugendlichen und Kindern zu beobachten. Bei herkömmlichen 3-piece-Intraokularlinsen ist bei physiologischer Stimulation keine Vorwärtsbewegung der Linse nachweisbar.

\section{Korrespondenzadresse \\ Prof. Dr. I. Lanzl

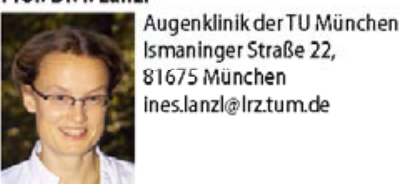

Danksagung. Für die Unterstützung sowie Bereitstellung der Geräte/Räume an das Team des AugenMVZKöld \& Partner Landshut für Anregungen zum Versuch is und fur die technisde Umsetzung bei Herm Oleg Kolliar.

Interessenkonflikt. Keine Angaben

\section{Literatur}

1. Atchison DA, Smith $G$ (2004) Possible errors in de termining axial length changes during accommodation with the IOLMaster. Optom Vis Sci 81: 283 286

2. Baikoff G, Lutun E, Ferraz C, Wei J (2004) Static and dynamic an alysis of the anterior segment with optical coherence tomography. J Cataract Refract Surg 30: 1843-1850

3. Beauchamp R. Mitchell B (1985) Ultrasound measures of vitreous chamber depth during oct lar accommodation. Am J Optom Physiol opt 62: 523-532

4. Charman WN, Heron G (1988) Fluctuations in accommodation: a review. Ophthalmic Physiol Opt 8 : 153-164

5. Connors R 3rd, Boseman P 3rd, Olson RJ (2002) Accuracy and reproducibility of biometry using partial coherence interferometry J Cataract Refract Surg 28: 235-238

6. Drexler W, FindI O, Schmetterer L et al. (1998) Eye 6. Drexler W, Findl $\mathrm{O}$, Schmetterer $\mathrm{L}$ et al. (1998) Eye ferences between emmetropes and myopes. Inferences between emmetropes and my
vest Ophthalmol Vis Sci 39: 2140-2147 vest Ophthalmol Vis Sci 39: 2140-2147 7. Garner LF, Smith G(1997) Changes in equivalent and gradient refractive index of the crystalline lens with accommodation. Optom 8. Garner LF, Yap MKH (1997) Changes in coular Ophthalmol Physiol Opt 17: 12-17

9. Jimenez R. Gonzalez MD, Perez MA, Gardia IA

(2003) Evolution of accommodative function and development of ocular movement in children. Ophthalmic Physiol Opt 23: 97-107

10. Kielhorn I, Rajan MS, Tesha PM et al. (2003) Clinica assessment of the Zeiss IOLMaster. J Cataract Refract Surg 29:518-522

11. Kirschkamp T, Dunne M, Barry JC (2004) Phako metric measurement of ocular sur face radii of ourvature, axial separations and alignment in relaxed and accommmodated human eyes. Ophthalmic Physiol Opt 24: 65-73
12. Klatt K, Langenbucher A, SeitzB, Nguyen NX (2006) Akkommodationsfahigkeit unter Einbeziehung refraktiver biometriscer und demogriscer meter. Ophthalmologe 103: 1032-1037

13. Koeppl C, Findl O, Kriechbaum K, Drexder W (2005) Comparison of pilocarpine-induced and stimulusdriven accommodation in phakic eyes. Exp Eye Res 80: 795-800

14. Koretz Jf, Cook CA, Kaufman PL (1997) Accommodation and presbyopia in the human eye. Changes in the anterior segment and cystalline lens with focus. Invest Ophthalmol Vis Sci 38: 569-578

15. Koretz IF, Cook CA Kaufman Pt (2001) Aging of the hum, She human lens. dangesin lens shape at zero-di opter accommodation. Jopt Soc Am A Opt Image

16. Kriechbaum K, Findl Q Kiss B et al. (2003) Compari son of anterior chamber depth measurement methods in phakic and pseudophakic eyes. J Cataract Refract Surg 29: 89-94

17. Kriechbaum K, Findl O, Koeppl Cet al. (2005) Stimulus-driven versus pilocarpine-induced biometric changes in pseudophakic eyes. Ophthalmology $112453-459$

18. Küchle $M, N g u y e n ~ N X$ Langenbucher A et al. (2002) Zwei Jahre Erfahrung mit der akkommodativen Hinterkammerlinse $1 \mathrm{Cu}$. Ophthalmologe 11: 820-824

19. Lam AK Chan R, Pang PC (2001) The repeatability and accuracy of axial length and anterior chamber depth measurements from the IOLMaster. Ophthalmic Physiol Opt 21: 477-483

20. Lesiewska-JunkH, Kaluzny J(2000) Intraocular lens movement and accommodation in eyes of youn patients. J Cataract Refract Surg 26: 562-565

21. Németh L, Fakete $\mathrm{O}$, Pesztenlehrer $\mathrm{N}$ (2003) Optical and ultrasound measurement of axial length and anterior chamber depth for in tr aocular fens power calculation. J Cataract Refract Surg 29:85-88

22. Rohen JW (1969) Akkommodationsapparat. In: Der Augenarzt, Bd. 1. Georg Thieme, Leipżg, S51-69

23. Rosenfield $M$, Portello JK, Blustein GH, Jang C (1996) Comparison of clinical techniques to assess the near accommodative response. Optom Vis Sci 73: $382-388$

24. Santodomingo-Rubido J, Mallen EA, Gilmartin B, Wolffsohn JS (2002) A new non-contact optical device for ocular biometry. Br JOphthalmol 86:458-462

25. Schneider $\mathrm{H}$, Stachs $\mathrm{O}$, Gobel K, GuthoffR (2006) Changes of the accommodative amplitude and the anterior chamber depth after implantation of an accommodative intraocular lens. Graefes Arch Clin Exp Ophthalmol 244: 322-329

26. Sheng $\mathrm{H}$, Bottjer CA, Bullimore MA (2004) Ocular component measurement using the Zeiss IOLMaster. Optom Vis Sci 81: 27-34

27. Shum PJ, Ko LS, Ng CL, Lin SL (1993) A biometric study of ocular changes during accommodation. Am JOphthalmol 115: 76-81

28. Stone RA, Quinn Gt, rrancistL et al. (2004) Diurnal axial length fluctuations in human eyes. Invest Ophthalmol Vis Sci 45: 63-70

29. Van der Heijde GL, Beers APA, Dubbelman $M$ (1996) Micofluctuations of steady-state accom modation measured with ultrasonography. Ophthalmic Physiol Opt 16: 216-221

30. Widk B, Gall R, Yothers T (2002) Cinical testing of accommodative facility: part III. Masked assess. ment of the relation between visual symptoms and binocular test results in school children and dults. Optometry 73: 173-181

31. Wold JE, Hu A, Chen S, Glasser A (2003) Subjec tive and objective measurement of human accom modative amplitude. J Cataract Refract Surg 29 : 1878-1888

\section{Der Ophthalmologe 2007}





\section{Appendix: Dokumentationsbögen}

\begin{tabular}{|l|}
\hline Nachname, Vorname: \\
\hline Geburtsdatum: \\
\hline Refraktionsanomalie: \\
\hline Datum der Messung: \\
\hline
\end{tabular}

1. Messung ohne Akkommodation

rechtes Auge
\begin{tabular}{|l||l|}
\hline $\mathrm{AL}$ & linkes Auge \\
$\mathrm{AL}$ & $\mathrm{AL}$ \\
$\mathrm{AL}$ & $\mathrm{AL}$ \\
$\mathrm{AL}$ & $\mathrm{AL}$ \\
MW & $\mathrm{AL}$ \\
\hline
\end{tabular}

rechtes Auge
\begin{tabular}{|l||l|}
\hline VKT & linkes Auge \\
VKT & VKT \\
VKT & VKT \\
VKT & VKT \\
MW & MW \\
\hline
\end{tabular}

2. Messung bei Nahakkommodation, Plättchen

rechtes Auge
\begin{tabular}{|l||l|}
$\mathrm{AL}$ & $\mathrm{AL}$ \\
$\mathrm{AL}$ & $\mathrm{AL}$ \\
$\mathrm{AL}$ & $\mathrm{AL}$ \\
$\mathrm{AL}$ & $\mathrm{AL}$ \\
MW & MW \\
\hline
\end{tabular}

\begin{tabular}{|l||l|}
\multicolumn{1}{l|}{ rechtes Auge } & linkes Auge \\
\hline VKT & VKT \\
VKT & VKT \\
VKT & VKT \\
VKT & VKT \\
MW & MW \\
\hline
\end{tabular}

\section{Messung mit Fernakkommodation, Plättchen}

rechtes Auge
\begin{tabular}{|l||l|}
\hline $\mathrm{AL}$ & linkes Auge \\
$\mathrm{AL}$ & $\mathrm{AL}$ \\
$\mathrm{AL}$ & $\mathrm{AL}$ \\
$\mathrm{AL}$ & $\mathrm{AL}$ \\
MW & MW \\
\hline
\end{tabular}

\begin{tabular}{l||l|} 
rechtes Auge & linkes Auge \\
\hline VKT & VKT \\
VKT & VKT \\
VKT & VKT \\
VKT & VKT \\
MW & MW \\
\hline
\end{tabular}

\section{Messung bei Nahakkommodation, Spiegel}

rechtes Auge
\begin{tabular}{|l||l|}
\hline $\mathrm{AL}$ & linkes Auge \\
$\mathrm{AL}$ & $\mathrm{AL}$ \\
$\mathrm{AL}$ & $\mathrm{AL}$ \\
$\mathrm{AL}$ & $\mathrm{AL}$ \\
MW & MW \\
\hline
\end{tabular}

rechtes Auge
\begin{tabular}{|l||l|}
\hline VKT & linkes Auge \\
VKT & VKT \\
VKT & VKT \\
VKT & VKT \\
MW & MW \\
\hline
\end{tabular}

\section{Messung bei Fernakkommodation, Spiegel}

rechtes Auge
\begin{tabular}{|l||l|}
\hline $\mathrm{AL}$ & linkes Auge \\
$\mathrm{AL}$ & $\mathrm{AL}$ \\
$\mathrm{AL}$ & $\mathrm{AL}$ \\
$\mathrm{AL}$ & $\mathrm{AL}$ \\
MW & MW \\
\hline
\end{tabular}

rechtes Auge
\begin{tabular}{|l||l|} 
VKT & linkes Auge \\
VKT & VKT \\
VKT & VKT \\
VKT & VKT \\
MW & VKT \\
\hline
\end{tabular}


Nachname, Vorname:

Geburtsdatum:

Refraktionsanomalie:

Datum der Messung:

1. AL ohne Akkommodation

\begin{tabular}{|l||l||l||l|}
\hline \multicolumn{2}{|c||}{ UNTERSUCHER 1 } & \multicolumn{2}{c|}{ UNTERSUCHER 2 } \\
rechtes Auge & Linkes Auge & rechtes Auge & linkes Auge \\
\hline \hline $\mathrm{AL}$ & $\mathrm{AL}$ & $\mathrm{AL}$ & $\mathrm{AL}$ \\
\hline $\mathrm{AL}$ & $\mathrm{AL}$ & $\mathrm{AL}$ & $\mathrm{AL}$ \\
\hline MW & MW & MW & MW \\
\hline
\end{tabular}

2. VKT ohne Akkommodation

\begin{tabular}{|l||l||l||l|}
\hline \multicolumn{2}{|c||}{ UNTERSUCHER 1 } & \multicolumn{2}{c|}{ UNTERSUCHER 2 } \\
rechtes Auge & Linkes Auge & rechtes Auge & linkes Auge \\
\hline \hline VKT & VKT & VKT & VKT \\
\hline VKT & VKT & VKT & VKT \\
\hline MW & MW & MW & MW \\
\hline
\end{tabular}

\section{AL bei Nahakkommodation, Plättchen}

\begin{tabular}{|l||l||l||l|}
\hline \multicolumn{2}{|c||}{ UNTERSUCHER 1 } & \multicolumn{2}{c|}{ UNTERSUCHER 2 } \\
rechtes Auge & Linkes Auge & rechtes Auge & AL \\
\hline \hline AL & AL & AL & AL \\
\hline AL & AL & AL & MW \\
\hline MW & MW & MW & \\
\hline
\end{tabular}

4. VKT bei Nahakkommodation, Plättchen

\begin{tabular}{|c|c|c|c|}
\hline \multicolumn{2}{|c|}{ UNTERSUCHER 1} & \multicolumn{2}{|c|}{ UNTERSUCHER 2} \\
\hline rechtes Auge & Linkes Auge & rechtes Auge & linkes Auge \\
\hline VKT & VKT & VKT & VKT \\
\hline VKT & VKT & VKT & VKT \\
\hline MW & MW & MW & MW \\
\hline
\end{tabular}

5. AL bei Nahakkommodation, Spiegel

\begin{tabular}{|c|c|c|c|}
\hline \multicolumn{2}{|c|}{ UNTERSUCHER 1} & \multicolumn{2}{|c|}{ UNTERSUCHER 2} \\
\hline rechtes Auge & Linkes Auge & rechtes Auge & linkes Auge \\
\hline$\overline{\mathrm{AL}}$ & $\overline{\mathrm{AL}}$ & 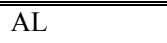 & $\overline{\mathrm{AL}}$ \\
\hline$\overline{\mathrm{AL}}$ & $\mathrm{AL}$ & $\mathrm{AL}$ & $\overline{\mathrm{AL}}$ \\
\hline MW & MW & MW & MW \\
\hline
\end{tabular}

6. VKT bei Nahakkommodation, Spiegel

\begin{tabular}{|l||l||l||l|}
\hline \multicolumn{2}{|c||}{ UNTERSUCHER 1 } & \multicolumn{2}{c|}{ UNTERSUCHER 2 } \\
rechtes Auge & Linkes Auge & rechtes Auge & linkes Auge \\
\hline \hline VKT & VKT & VKT & VKT \\
\hline VKT & VKT & VKT & VKT \\
\hline MW & MW & MW & MW \\
\hline
\end{tabular}

